Aus der Abteilung Präventive Zahnmedizin, Parodontologie und Kariologie

(Komm. Leiter: Prof. Dr. med. dent. M. Hülsmann)

im Zentrum Zahn-, Mund- und Kieferheilkunde

der Medizinischen Fakultät der Universität Göttingen

\title{
Vergleich der Farbstabilität milder und hochkonzentrierter Bleichmittel
}

Eine In-vitro-Untersuchung

\author{
INAUGURAL - DISSERTATION \\ zur Erlangung des Doktorgrades für Zahnheilkunde \\ der Medizinischen Fakultät der \\ Georg-August-Universität zu Göttingen \\ vorgelegt von \\ MONIKA MARIA REUS \\ aus \\ Fulda
}

Göttingen 2009 
Dekan: $\quad$ Prof. Dr. med. C. Frömmel

I. Berichterstatter: Prof. Dr. med. dent. R. Mausberg

II. Berichterstatter/in:

III. Berichterstatter/in:

Tag der mündlichen Prüfung: 


\section{Inhaltsverzeichnis}

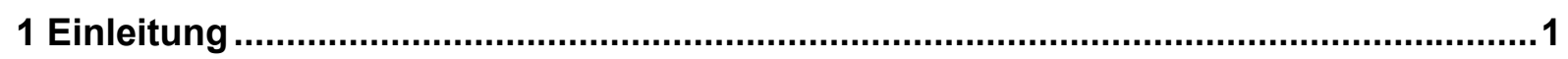

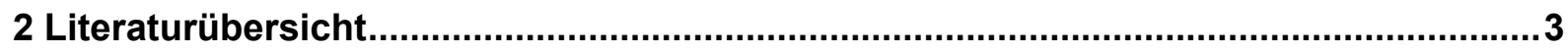

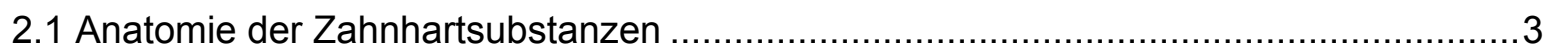

2.1.1 Anatomie und Histologie des Schmelzes ........................................................ 3

2.1.2 Anatomie und Histologie des Dentins........................................................ 4

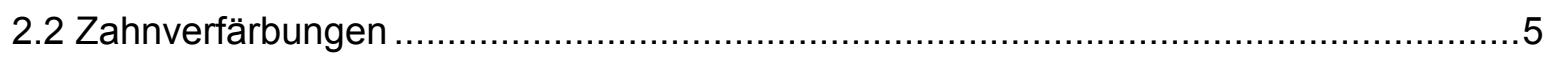

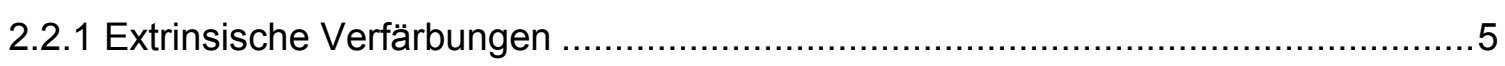

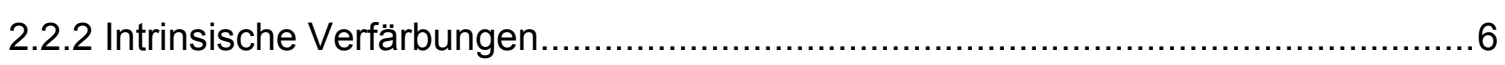

2.2.2.1 Präeruptive Zahnverfärbung (und deren Ursachen) ..................................6

2.2.2.2 Posteruptive Zahnverfärbung (und deren Ursachen) ....................................

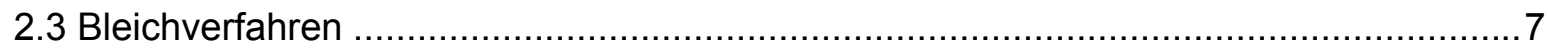

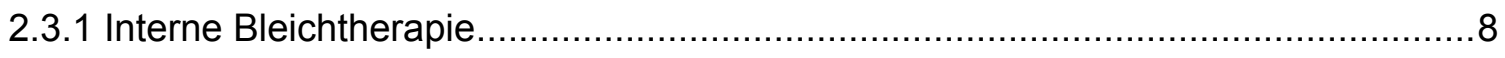

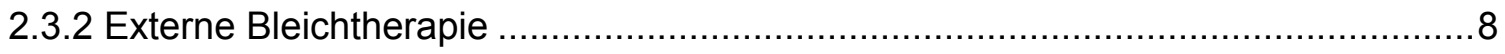

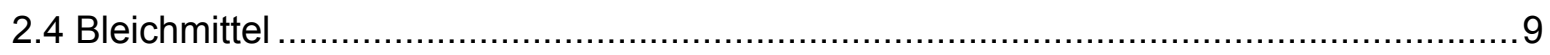

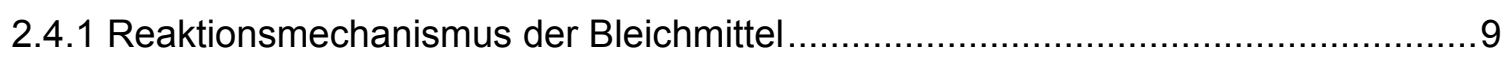

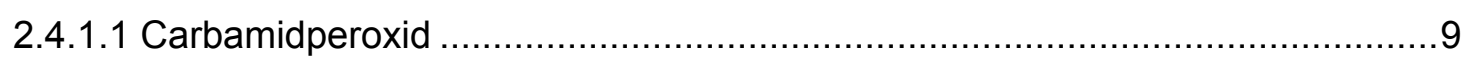

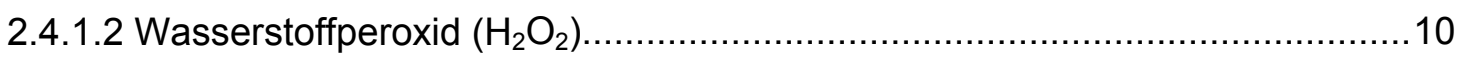

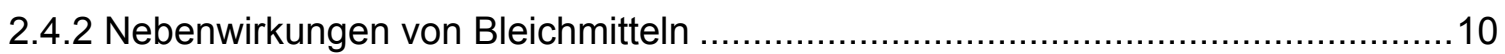

2.5 Wahrnehmung und Bestimmung der Zahnfarbe ......................................................11

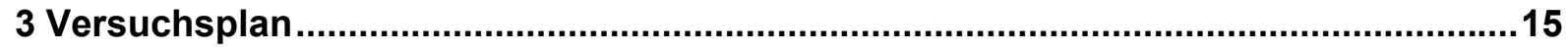

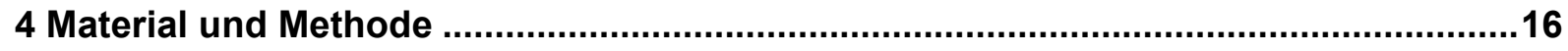

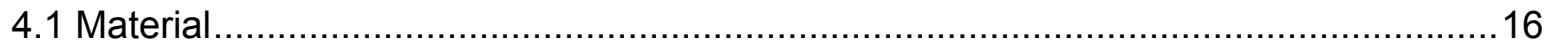

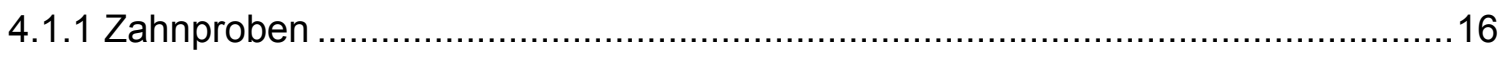

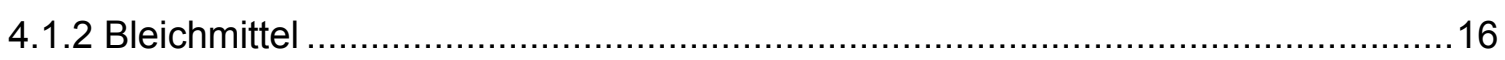

4.1.3 Versuchsapparatur zur Herstellung standardisierter digitaler Fotos..................... 17

4.1.4 Kamerasystem und Einstellungen ........................................................ 18

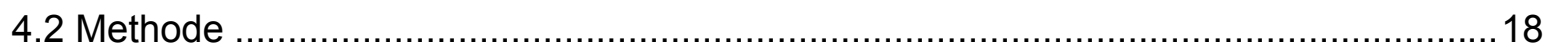

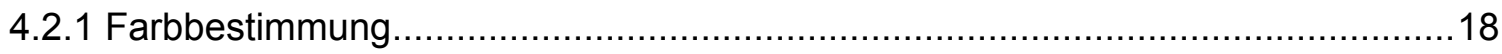

4.2.2 Randomisierung und Gruppeneinteilung (Kontrollgruppe, PF 15\% und XtraBoost)

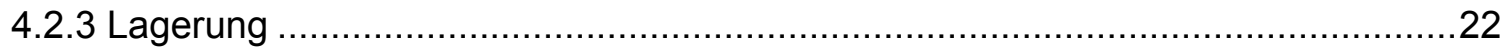

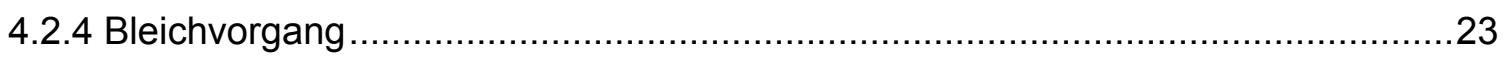

4.2.5 Farbbestimmung während des Studienverlaufs .........................................24

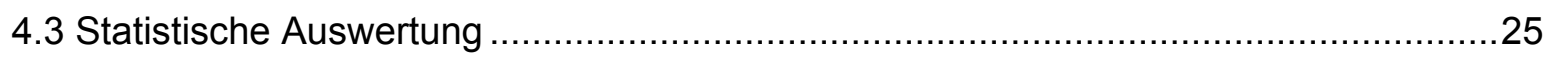

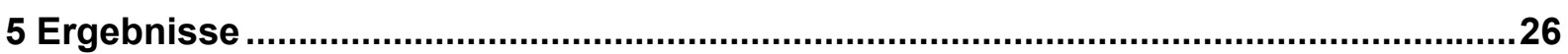




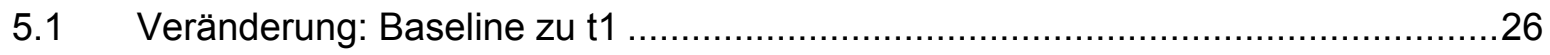

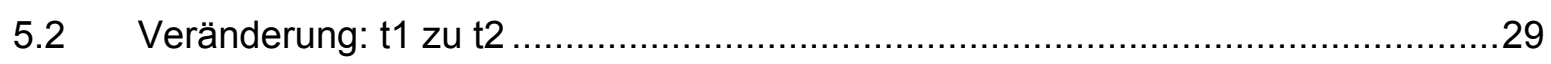

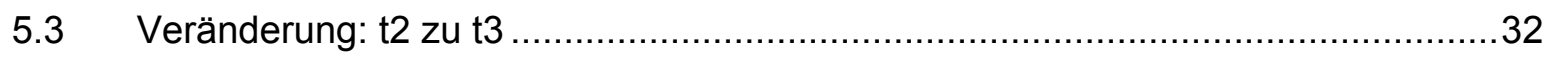

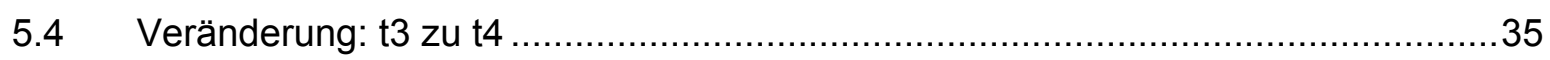

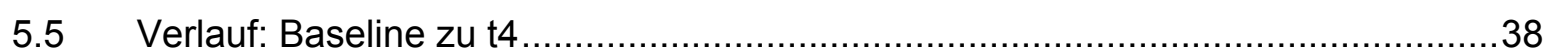

5.6 Gruppenvergleich über den zeitlichen Verlauf................................................. 44

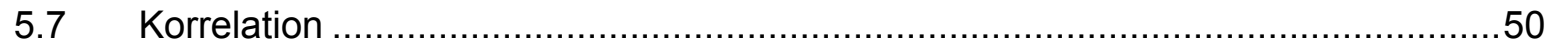

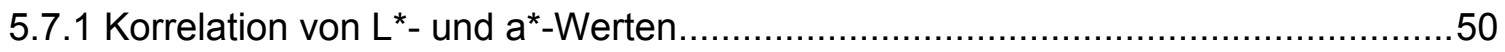

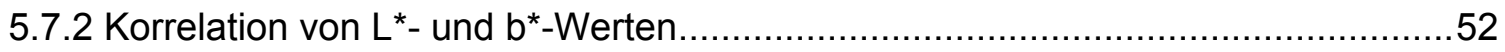

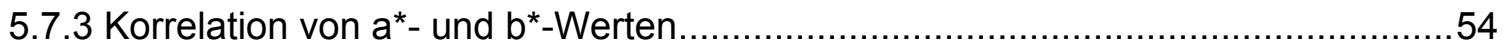

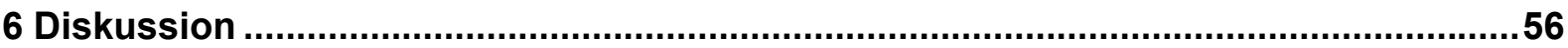

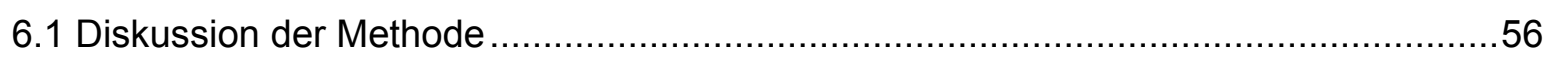

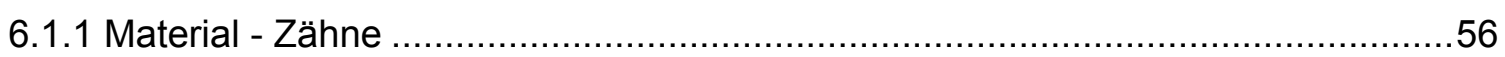

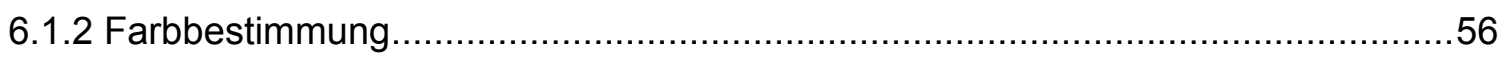

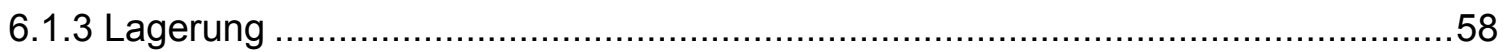

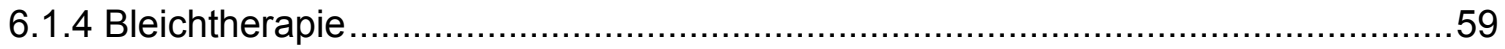

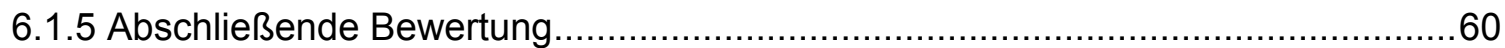

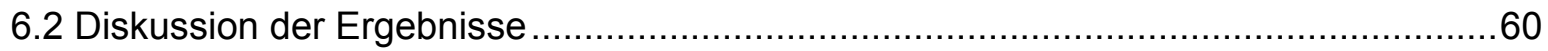

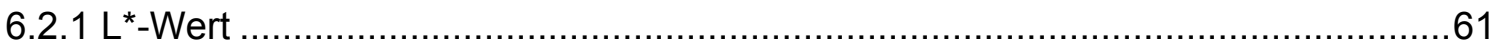

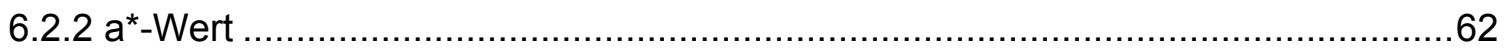

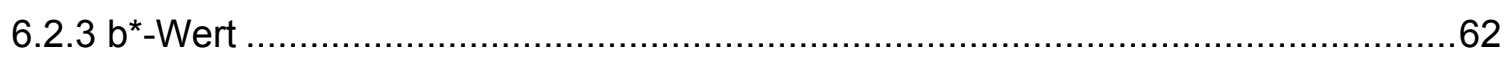

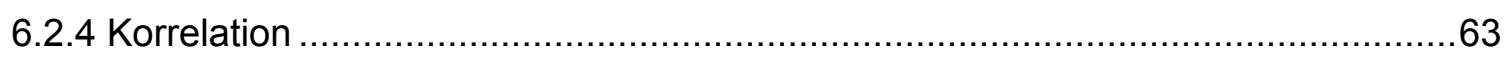

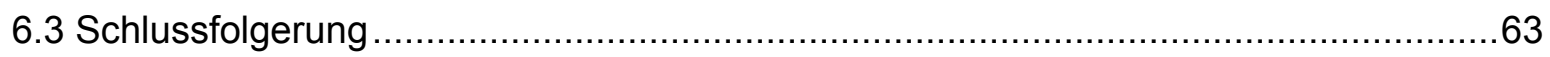

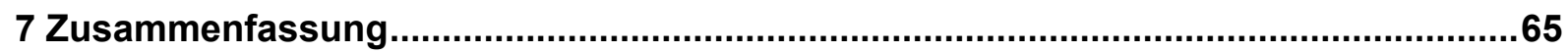

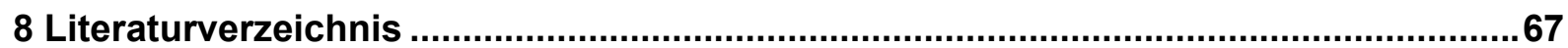

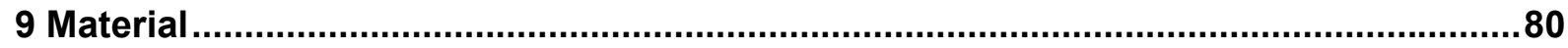

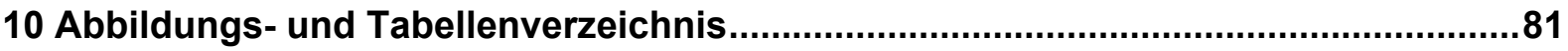

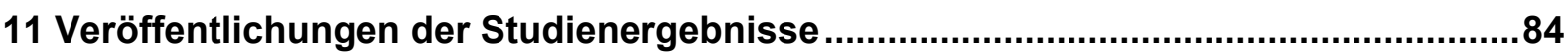




\section{Einleitung}

Zahnfehlstellungen und Zahnverfärbungen haben einen zunehmenden Einfluss auf das äußere Erscheinungsbild des Menschen. Damit gewinnt heutzutage die Ästhetik in der Zahnheilkunde immer mehr an Bedeutung (Joiner 2006). Das Schönheitsempfinden an sich unterliegt dabei dem soziokulturellen Umfeld. Während hierzulande weiße, ebenmäßige Zähne ohne Fehlstellung ein Schönheitsideal darstellen, sind zum Beispiel Goldkronen im Frontzahnbereich in vielen islamischen Ländern immer noch ein Symbol für Wohlstand und Reichtum. In Japan galt lange Zeit das Schwärzen von Zähnen als Zeichen einer Vermählung (Strub et al. 2005a). In unserer modernen Gesellschaft stehen makellose weiße Zähne für Gesundheit, Attraktivität und damit letztlich auch unterschwellig für Leistungsfähigkeit, Erfolg und hohen Lebensstandard. Nicht zuletzt aus diesem Empfinden heraus gewinnt das Bleichen als Therapie für verfärbte Zähne zunehmend an Bedeutung und wird von den Patienten immer öfter gefordert (Maibach-Nagel 2000).

Die Ursachen von verfärbten Zähnen sind vielfältig und durch eine zahnmedizinische Anamnese im Rahmen eines Patientengespräches zu diagnostizieren (Attin 2002). Dabei bilden fundierte Kenntnisse über die Wirkungsweise der verschiedenen Bleichmittel sowie deren Nebenwirkungen die Basis für eine erfolgreiche Bleichtherapie.

Prinzipiell unterscheidet man zwei Aufhellungstherapien: internes und externes Bleichen (Severcan et al. 2008). Beim internen Bleichen wird das Bleichmittel unmittelbar in die Pulpakammer von wurzelkanalbehandelten und intrinsisch verfärbten Zähnen eingebracht und dort für einige Tage belassen. Beim externen Bleichen dagegen werden die Zähne von auBen mit einem aufgebrachten Bleichgel behandelt.

Externes Bleichen kann mit Hilfe mannigfacher Verfahren durchgeführt werden. Neben den beiden gebräuchlichsten, dem so genannten Home-Bleaching und der In-Office-Methode, gibt es noch eine Reihe von Bleichverfahren, die mit frei verkäuflichen Präparaten durchgeführt werden. Hierzu zählen zum Beispiel das Paint on varnish (Vita Style Paint ${ }^{\circledR}$ ), Paint on lacquer (Colgate-simply-White ${ }^{\circledR}$ ) oder diverse Whitening strips (blend-a-med White Strips ${ }^{\circledR}$, Crest White Strips Supreme ${ }^{\circledR}$ ) (Mielczarek et al. 2008, Kielbassa et al. 2009).

Das Home-Bleaching wird vom Patienten selbst durchgeführt. Mit Hilfe von individuell angepassten Schienen wird das Bleichmittel (zum Beispiel 10-15\%iges Carbamidperoxid) auf die Zähne appliziert und kann so über mehrere Stunden tagsüber oder über Nacht einwirken (Wiegand und Attin 2002).

Beim so genannten In-Office oder Chairside-Bleaching erfolgt das Bleichen direkt am Behandlungsstuhl durch den Zahnarzt. Es ist eine intensive und schnelle Methode zur Zahnaufhellung und besonders geeignet für die Aufhellung einzelner Zähne. Die dabei benutzten hoch konzentrierten Bleichmittel (35\%iges Carbamidperoxid) werden unter Kofferdameinsatz 
direkt auf den Zahn aufgetragen und gegebenenfalls anschließend durch UV-Licht aktiviert (Lips 2004).

Der erreichte Bleicheffekt ist allerdings nicht in allen Fällen von Dauer. So konnte ein Rückgang der erwirkten Helligkeit der Zähne in Einzelfällen bis hin zum Ausgangszustand nachgewiesen werden. In der Studie von Zanter et al. (2006) waren die Bleichergebnisse etwa 6 Monate stabil, wohingegen in der Studie von Leonard (2000) sich auch nach 3 Jahren noch bei $63 \%$ der Patienten und nach 7 Jahren immer noch bei $42 \%$ der Patienten ein zufrieden stellender Bleicheffekt zeigte.

Der angestrebte bzw. erreichte Bleicheffekt, die Kontrolle seiner Nachhaltigkeit und somit die Bewertung des Bleichverfahrens erfordert eine sichere Farbbestimmung, die bis dato in der Regel durch einen visuellen Vergleich mit Farbringen durchgeführt wird (Baumann und Schifferdecker 1994, Musil und Kittler 1983, van der Burgt et al. 1985). Jedoch zeigt dieses subjektive Verfahren einige methodische Schwächen, da die Farbwahrnehmung von vielerlei Parametern beeinflusst wird. Dabei spielen insbesondere äußere Faktoren wie Umgebungshelligkeit, Lichtintensität und Lichtfarbe eine Rolle, wie auch das physische und psychische Befinden des Betrachters selbst. Auch die Dauer der Betrachtung beeinflusst das Helligkeitsund Farbempfinden (Strub et al. 2005b). Eine Farbbestimmung durch einen visuellen Vergleich ist also durchaus problematisch und in gewissem Maße subjektiv. Sie ist nur bedingt reproduzierbar und daher auch nur bedingt wissenschaftlich einsetzbar (Culpepper 1970).

Vor diesem Hintergrund stellte sich die Frage, wie man die Farbbestimmung beim Bleichen von Zähnen objektivieren kann. Eine Chance hierzu eröffnet der Einsatz der modernen Digitalfotografie mit der Möglichkeit der pixelweisen Bildauslese und Bildbearbeitung. Mit Hilfe von digitalen Fotos und einem kommerziellen Computerprogramm, zum Beispiel Adobe Photoshop CS (Adobe Systems GmbH, München, Germany), kann die subjektive Betrachtungsweise durch eine objektive Farbanalyse per Computer ersetzt werden (Bengel 2003, Bentley et al. 1999).

Ziel dieser Studie war es, unter Erprobung dieser computergestützten Farbanalyse festzustellen, ob es Unterschiede im Erhalt der Farbstabilität zwischen niedrig konzentrierten (15\%iges Carbamidperoxid) und hoch konzentrierten (35\%iges Carbamidperosid) Bleichmitteln beziehungsweise zwischen den beiden verschiedenen Bleichmethoden Home-Bleaching und In Office-Bleaching gibt. Dazu wurden 231 extrahierte menschliche Front- und Eckzähne auf drei Gruppen (mit Kontrollgruppe) aufgeteilt. Nach entsprechender Bleichung wurden die Zahnproben über einen Untersuchungszeitraum von sechs Monaten in bestimmten Zeitintervallen mit Hilfe von standardisierten digitalen Zahnfotos und einem Analyseprogramm farblich ausgewertet. Abschließend wurden die gewonnenen Datensätze statistisch auf signifikante Änderungen ausgewertet. 


\section{Literaturübersicht}

\subsection{Anatomie der Zahnhartsubstanzen}

Grundlage für das Verständnis der Entstehung von Zahnverfärbungen sowie deren Beseitigung ist die Anatomie des Zahnes. Schmelz bildet zusammen mit dem Dentin und dem Wurzelzement die mineralisierte Zahnhartsubstanz. Diese umschließt das Weichgewebe, die Pulpa (Abbildung 2.1).

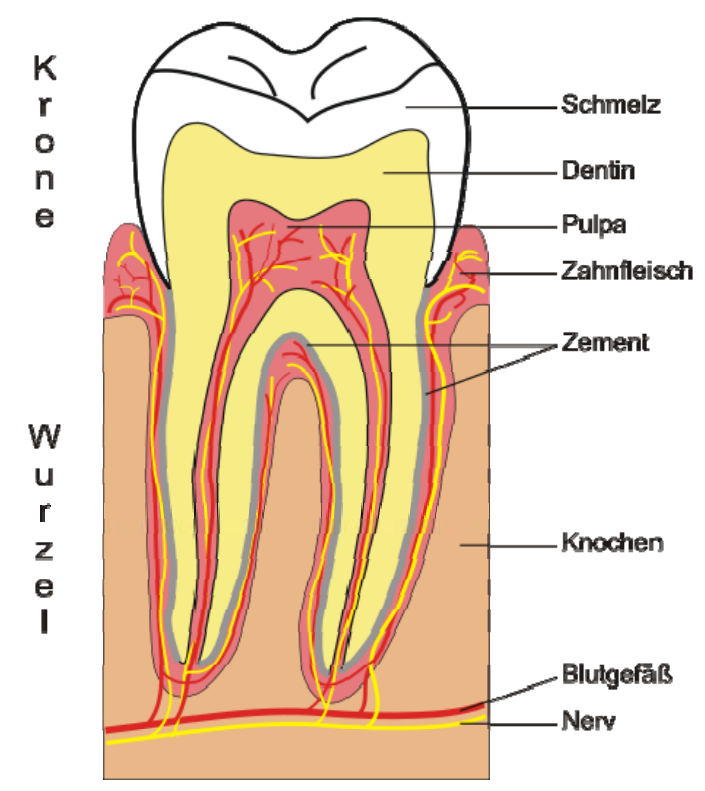

Abbildung 2.1: $\quad$ Anatomie eines Zahns sowie seiner umliegenden Strukturen (Qulle:http://upload.wikimedia.org/wikipedia/commons/thumb/d/ dc/Schematic_section_tooth.svg/150pxSchematic_ section_tooth.svg.png)

\subsubsection{Anatomie und Histologie des Schmelzes}

Der Zahnschmelz ist die härteste Substanz im menschlichen Körper und bedeckt die Zahnkrone (Hellwig et al. 2006). Er wird von Ameloblasten gebildet und besteht zu 93-98 Gew\% aus anorganischen Substanzen wie Kalzium, Karbonat, Magnesium und Natrium. Die restlichen 5 Gew\% sind Wasser (1,5-4 Gew\%) und organische Bestandteile wie Proteine und Lipide (Hellwig et al. 2006). Sein kristallines Gefüge besteht im Verhältnis von 1:1,2 aus einer Kalzium-Phosphat-Verbindung, dem so genannten Apatit $\left(\mathrm{Ca}_{10-x} \mathrm{PO}_{6-\mathrm{x}}\right) \times \mathrm{X}_{2} \times \mathrm{H}_{2} \mathrm{O}_{2}$. Die 
Apatitkristalle sind im Querschnitt annähernd hexagonal und $160 \mathrm{~nm}$ lang, 40-70 nm breit sowie $26 \mathrm{~nm}$ dick (Hellwig et al. 2006). Diese Werte schwanken je nach Lokalisation und Reifegrad des Schmelzes. Etwa 100 Schmelzkristalle organisieren sich zu so genannten Schmelzprismen, die sich von der Schmelz-Dentin-Grenze bis fast zur Zahnoberfläche wellenförmig erstrecken. Diese Prismen erscheinen im Querschnitt in verschiedenen Formen. Die häufigsten Prismenkonfigurationen sind der Schlüssellochtyp, der Hufeisentyp und der zylindrische Typ (Höhling 1966). Zahnschmelz ist aufgrund seines hohen Anteils an anorganischen Bestandteilen weitestgehend transluzent, hat also keine ausgeprägte Eigenfarbe. Die Schmelzhärte beträgt im Schnitt zwischen 390 KHN (Knoop-hardness numbers) an der Oberfläche und $250 \mathrm{KHN}$ an der Schmelz-Dentin-Grenze (Hellwig et al. 2006).

\subsubsection{Anatomie und Histologie des Dentins}

Dentin (Zahnbein) stellt die Hauptmasse des humanen Zahns dar. Das koronale Dentin ist von Schmelz und das apikale Dentin von Zement umgeben. Es besteht zu $70 \mathrm{Gew} \%$ aus anorganischem und zu $20 \mathrm{Gew} \%$ aus organischem Material. Die restlichen $10 \mathrm{Gew} \%$ sind Wasser (Schroeder 1992). Den Großteil der anorganischen Substanz machen - wie beim Schmelz - Phosphat und Kalzium aus. Die organische Matrix besteht zum größten Teil aus Kollagen (91-92\%). Dabei stellt das Typ-I-Kollagen den überwiegenden Prozentanteil, Typ-VKollagen ist nur zu weniger als $3 \%$ enthalten und Typ-III-Kollagen ist kein Bestandteil im Dentin (Butler 1984). Dentin ist weniger hart als Schmelz und besitzt zudem eine Eigenfarbe von hellem Gelb bis Braun. Das Dentin wird von Odontoblasten gebildet, deren Zellkörper sich in der Pulpa befinden. Die Zellfortsätze der Odontoblasten, die Tomes'schen Fasern, liegen in Dentinkanälchen, umhüllt von Dentinliquor. Diese Dentinkanälchen (Tubuli) sind ein typisches Strukturmerkmal des Dentins (Strub et al. 2005a), ihre Dichte (Anzahl/ $\mathrm{mm}^{2}$ ) nimmt von 43.000 pulpanah auf 19.000 pulpafern ab. Auch die Durchmesser der Tubuli sinken mit zunehmender Entfernung von der Pulpa von 1,9 $\mu \mathrm{m}$ auf etwa 0,8 $\mu \mathrm{m}$ (Garberoglio und Brännström 1976). Dentin wird während der gesamten Lebensdauer eines Zahnes gebildet, bis zum Abschluss des Wurzelwachstums entsteht Primärdentin, später wird von der Pulpaseite her reguläres Sekundärdentin angebaut. Als umlaufenden Schutz der Pulpa unterscheidet man noch das so genannte Tertiärdentin (Reizdentin, irreguläres Sekundärdentin, Reparationsdentin), welches aufgrund von lokalen Reizen (zum Beispiel Karies, Erosion oder Attrition) an der Pulpa-Dentin-Grenze gebildet wird und oft eine dunklere Farbe aufweist (Hellwig et al. 2006). 


\subsection{Zahnverfärbungen}

Zahnverfärbungen lassen sich anhand ihrer Genese in extrinsische und intrinsische Verfärbungen unterteilen. Sie können angeboren oder erworben sein (Attin 2002).

\subsubsection{Extrinsische Verfärbungen}

Gesunde, das heißt karies- und füllungsfreie, saubere Zähne verfärben sich kaum. Erst die auf dem Schmelz befindliche organische Matrix, bestehend aus acquired Pellikel (erworbenes Schmelzoberhäutchen) oder Plaque (bakterielle Beläge), fördert die Anlagerung von Farbstoffen oder farbstoffbildenden Substanzen an die Zahnhartsubstanz (Schroeder 2000). Vor allem durch Nahrungs- und Genussmittel wird diese organische Matrix verfärbt. Besonders relevant sind in diesem Zusammenhang etwa Tee und Kaffee, welche färbende Polyphenole enthalten. Rotwein und Traubensaft enthalten unter anderem das färbend wirkende Tannin. Auch Teerkondensate aus Tabakwaren sowie Farbstoff enthaltende Gewürze, Gemüse und Beerensorten können zu Verfärbungen führen (Attin 2002).

Weiterhin können oral applizierte eisen- oder nitrathaltige Medikamente oder Chemotherapeutika wie zum Beispiel Mundspüllösungen, die Chlorhexidindigluconat oder Zinnfluoride enthalten, Verfärbungen hervorrufen. Nicht zuletzt treten bei mangelnder Mundhygiene chromogene Bakterien auf, die nicht nur zu Karies und Parodontitis, sondern auch zur Veränderung der Qualität des Zahnbelags führen können (Attin 2002).

Den Nathoo-Klassifikationen (N1-N3) nach lassen sich externe Verfärbungen wie folgt einteilen (Nathoo 1997):

- N1: Chromogene (farbgebende Substanzen), deren Farbwirkung auf konjugierten Doppelbindungen basiert. Sie lagern sich an die Zahnoberfläche an und behalten ihren Farbton bei. Chromogene wie z.B. Tannin oder Polyphenole sind z.B. in Tee, Kaffee, Rotwein oder Tabakrauch enthalten. Zu dieser Gruppe zählen auch die farbtragenden Metallverbindungen.

- N2: Chromogene, die nach Anlagerung an der Zahnoberfläche oder deren Pellikel eine Verfärbung durch chemische Modifikation der Pellikelproteine erzeugen. Hierzu gehören z.B. bakterielle Beläge (Erikson und Nordbo 1978).

- N3: Prächromogene (primär farblose Substanzen), die durch physikalisch-chemische Prozesse in einen farbgebenden Komplex umgewandelt werden. Beispiele hierfür sind Chlorhexidindigluconat oder die bekannte Apfelbräune an der Luft. 
Hiervon zu unterscheiden sind einfache oberflächige Verfärbungen. Diese lassen sich in der Regel durch eine professionelle Zahnreinigung beseitigen und durch Prophylaxeunterweisungen vermeiden und stellen noch keine Indikation für ein Bleichverfahren dar (DGZMK 2000).

\subsubsection{Intrinsische Verfärbungen}

Intrinsische oder interne Verfärbungen entstehen durch Einlagerungen in die Zahnhartsubstanz. Auch sie können - wie die externen Verfärbungen- verschiedener Genese sein. So können sich Struktur- und Dimensionsänderungen der Zahnhartsubstanz durch Erkrankungen, erbliche Anomalien, Traumata oder durch das Alter entwickeln. Generell unterscheidet man interne Verfärbungen nach ihrem Entstehungszeitraum, als präeruptive Verfärbungen, wenn sie bereits während der Zahnentwicklung angelegt sind, und als posteruptive Verfärbungen, wenn sie erst nach dem Zahndurchbruch auftreten (Attin 2002).

\subsubsection{Präeruptive Zahnverfärbung (und deren Ursachen)}

Systemisch wirkende Noxen und Mangelzustände, wie zum Beispiel Hypovitaminosen der Vitamine A, C (Skorbut) und D (Rachitis) sowie Kalzium- und Phosphatmangel, sind verantwortlich für Schmelzhypoplasien (Stöckli und Ben-Zur 1994). Ähnliche Erscheinungen treten auch bei der genetisch bedingten Amelogenesis imperfecta und Dentinogenesis imperfecta auf (Ernst et al. 1995). Des Weiteren können auch Allgemeinerkrankungen wie chronische Diarrhöe, Lues, Rubeola oder Salmonellose Ursache für Mineralstoffwechselstörungen sein. Die systemische Gabe von Tetrazyklin oder dessen Derivaten als Antibiotikum während der Zahnentwicklung (2. Schwangerschaftstrimenon bis hin zum achten Lebensjahr) können ebenfalls ursächlich für massive Verfärbungen sein (Schroeder 1997, Weyman 1965 a, Glockner et al. 1997). Dabei bilden Tetrazykline mit Kalzium gelbliche Komplexe, die während der Schmelzmineralisation irreversibel in das Hydroxylapatit eingelagert werden. Je nach Art des verabreichten Tetrazyklins und der Dauer der Behandlung schwanken die Verfärbungen zwischen grau und braun. Häufig betroffen sind - in Abhängigkeit von Konzentration und Zeitpunkt der Medikamentengabe - einzelne Zahnpartien, manchmal auch die gesamte Zahnkrone (Weyman 1965 a).

Schmelzveränderungen an einzelnen Zähnen der zweiten Dentition können durch Traumata an den Milchzähnen entstehen, die sich auf die darunter liegenden Zahnkeime auswirken (Turner-Zahn) (Zanter et al. 2005).

Wenn einem Kind zwischen dem 2. Schwangerschaftstrimester und dem 9. Lebensjahr mehr als 1 ppm Fluorid täglich zugeführt wird, können Fluorosen während der Zahnentwicklung 
entstehen. Klinisch stellen sich diese als kreidig weißliche bis dunkelbraune Verfärbung dar (Geurtens und Vernieks1986, Glockner et al.1997).

\subsubsection{Posteruptive Zahnverfärbung (und deren Ursachen)}

Im Alter kann sich die Zahnfarbe aufgrund von zunehmender Sklerosierung des Dentins und Obliteration der Pulpa durch Tertiär- und Sekundärdentinbildung verändern. Die Zähne verlieren dabei zunehmend ihre Transluzenz, werden opaker und erscheinen dunkler (Attin und Kielbassa 1995, Goldstein und Garber 1995). Auch durch zahnärztliche Maßnahmen können Farbveränderungen hervorgerufen werden, so können Amalgamfüllungen oder Silberstifte Metalloxide freisetzen, die eine dunkle Färbung hervorrufen (Feinman et al. 1987). Der gleiche Effekt lässt sich auch bei Nichtedelmetallgerüsten keramisch verblendeter Kronen, vor allem im Zahnhalsbereich überkronter Frontzähne, beobachten. Auch Wurzelfüllmaterialien und Medikamente wie Eugenol-Zement, N2 Universal ${ }^{\circledR}$, Diaket ${ }^{\circledR}$, Tubli-Seal ${ }^{\circledR}$, Dubler Paste ${ }^{\circledR}$ oder Endomethasone und Ledermix ${ }^{\circledR}$ können nach endodontischer Behandlung intrinsische Verfärbungen hervorrufen (Kim et al. 2000, Plasschaert und van der Burgt 1988).

Geraten Blut- oder Gewebereste in die Pulpenkammer, zum Beispiel durch Pulpanekrosen, Traumata oder nicht sorgfältige Aufbereitung bei Wurzelkanalbehandlung, setzen sie bei ihrem Abbauprozess das im Hämoglobin eingebaute Eisen frei. Dieses kann mit Schwefel aus nekrotischen Proteinen oder Bakterienstoffwechselprodukten zu Eisensulfid reagieren, welches dem Zahn dann eine gräuliche Färbung verleiht (Glockner et al. 1997, Guldener und Langeland 1987). Aktive kariöse Läsionen besitzen ebenfalls die Fähigkeit Zähne intern zu verfärben. Dabei können sich bei Remineralisationsvorgängen exogene Farbstoffe einlagern, wodurch eine ruhende/inaktive Schmelzkaries braungelb erscheint (Heidemann 1999).

\subsection{Bleichverfahren}

Oberflächige, externe Zahnverfärbungen werden in der Regel durch eine professionelle Zahnreinigung mit Polierkelchen, Bürsten, Pasten oder Pulverstrahlgeräten beseitigt. Solche Belagsverfärbungen bedürfen in der Regel keiner speziellen Bleichtherapie. Jedoch hat die Zahnreinigung dann ihre Grenzen, wenn tief in die Zahnhartsubstanz eingedrungene Verfärbungen oder Pigmenteinlagerungen so nicht entfernt werden können. Daher kann auch bei solch tief liegenden externen Zahnverfärbungen eine Bleichtherapie indiziert sein. Zahnaufhellungen werden mit den bleichenden Substanzen Carbamid- oder Wasserstoffperoxid durchgeführt. Im Gegensatz zu restaurativen Versorgungen wie Kronen, Inlays oder Veeners haben sie sich als minimalinvasive und Zahnhartsubstanz schonende Alternative durchge- 
setzt (Sarrett 2002). Grundsätzlich lassen sich zwei Bleichtherapien unterscheiden, die interne und die externe Aufhellungstherapie.

\subsubsection{Interne Bleichtherapie}

Die interne Bleichtherapie, auch Walking-Bleach-Technik genannt, beschränkt sich auf die Behandlung von avitalen Zähnen. Hierbei wird das Bleichmittel direkt in der Pulpakammer des wurzelkanalbehandelten, verfärbten Zahnes appliziert (Attin et al. 2003). Das Bleichmittel verbleibt dort für drei bis fünf Tage, wobei das Cavum mit einer dichten provisorischen Füllung verschlossen wird. Dieser Vorgang kann mehrmals wiederholt werden, jedoch sollte sich nach drei bis vier Zyklen ein Bleicherfolg eingestellt haben (Rotstein et al. 1991). Als Bleichsubstanz wird beim internen Bleichen Natriumperborat verwendet, welches mit Wasser oder $3 \%$ igem $\mathrm{H}_{2} \mathrm{O}_{2}$ angemischt wird. Höhere $\mathrm{H}_{2} \mathrm{O}_{2}$-Konzentrationen (zum Beispiel $30 \%$ iges $\mathrm{H}_{2} \mathrm{O}_{2}$ ) haben sich nicht durchgesetzt, da mit ihnen ein erhöhtes Nebenwirkungsrisiko, wie zum Beispiel zervikale Resorptionen, assoziiert ist (Rotstein et al. 1991). Kontraindikationen für eine interne Bleichtherapie stellen ausgedehnte Füllungen, Schmelzfrakturen oder insuffiziente Wurzelkanalfüllungen dar (Glockner et al. 1995, Russe et al. 1990). Das interne Bleichverfahren erzielt ästhetisch gute Ergebnisse (Feiglin 1987, Freidman et al. 1988, Howell 1980) und bei richtiger Durchführung und Dosierung lassen sich Spätfolgen und Nebenwirkungen minimieren (Glockner et al. 1997).

\subsubsection{Externe Bleichtherapie}

Externe Bleichverfahren werden danach unterschieden, wo sie durchgeführt werden. Beim In-Office-Verfahren findet das Bleaching in der Zahnarztpraxis (Chairside) statt. Wird der Bleichvorgang vom Patienten selbst zu Hause durchgeführt, spricht man vom HomeBleaching. Bei beiden Verfahren sollte vor Beginn der Behandlung eine professionelle Zahnreinigung sowie eine Kontrolle auf kariöse Läsionen erfolgen. Freiliegende Zahnhälse werden dabei mit einem Dentinhaftvermittler versiegelt, um das Eindringen des Bleichmittels in die Dentintubuli zu verhindern und somit Pulpaschäden vorzubeugen.

Beim In-Office-Bleaching wird unter Kofferdamschutz hochkonzentriertes (> 30\%iges) Carbamidperoxidgel durch den Zahnarzt auf die zu bleichenden Zähne appliziert (Lips 2004). Durch thermokatalytische Verfahren kann die Wirkung der Bleichmittel gesteigert werden (Luk et al. 2004), dabei ist allerdings zu beachten, dass Licht und Hitze während des Bleichverfahrens zu Pulpaschädigungen durch Temperaturerhöhungen im Zahninneren führen können (Baik et al. 2001, Luk et al. 2004). Bei mangelnder Erfahrung sollte in jedem Fall auf ein beschleunigendes thermokatalytisches Bleichverfahren verzichtet und einer behut- 
sameren Bleichtherapie der Vorzug gegeben werden (Auschill et al. 2005, Tredwin et al. 2006).

Beim Home-Bleaching wird das Carbamidperoxid- oder Wasserstoffperoxidgel (10-15\%) mit Hilfe einer laborgefertigten angepassten Schiene durch den Patienten selbst auf die zu bleichenden Zähne appliziert. Der Patient muss bei diesem Verfahren lediglich zur Beratung und Instruktion, zur Abdrucknahme und zur Anpassung der Schiene (um Irritationen der Gingiva zu vermeiden) die Zahnarztpraxis aufsuchen. Die Schiene wird in der Regel über einen Zeitraum von 10 -15 Tagen getragen, wobei die tägliche Anwendung zwischen 30 Minuten und mehreren Stunden (beim Tragen über Nacht), variieren kann (Wiegand und Attin 2002). Neben diesen vom Zahnarzt durchgeführten beziehungsweise begleiteten Bleichtherapien gibt es noch eine Vielzahl freiverkäuflicher Bleichpräparate auf dem Markt (Sarrett 2002). Hierzu zählen zum Beispiel das Paint on-varnish-Verfahren (Viva Style Paint ${ }^{\circledR}$ ), Paint on-lacquerVerfahren (Colgate-simply-White ${ }^{\circledR}$ ) oder die Whitening strips (blend-a-med White Strips ${ }^{\circledR}$, Crest White Strips Supreme ${ }^{\circledR}$ ) (Mielczarek et al. 2008, Kielbassa et al. 2009). Diese Folien und Gelees werden vom Patienten selbst auf die Ober- und Unterkieferfrontzähne appliziert beziehungsweise geklebt. Hinsichtlich der Wirksamkeit zeigen diese freien Verfahren keinen Unterschied gegenüber der herkömmlichen Schienentherapie mit Carbamidperoxid (Gerlach und Zhou 2001, Gerlach und Zhou 2002, Kielbassa 2009).

\subsection{Bleichmittel}

In der Zahnheilkunde werden hauptsächlich die aufhellenden Substanzen Carbamidperoxid und Wasserstoffperoxid verwendet. Das Bleichen von Zähnen mit Hilfe dieser peroxidbasierten Aufheller beruht auf einer Oxidationsreaktion mit den färbenden organischen Substanzen, die sich in oder auf den Zähnen gebildet beziehungsweise abgelagert haben (Goldstein und Garber 1995).

\subsubsection{Reaktionsmechanismus der Bleichmittel}

\subsubsection{Carbamidperoxid}

Bei Carbamidperoxid handelt es sich um Harnstoff mit einer Peroxidgruppe, welche in wässriger Lösung reaktiven Sauerstoff abgibt. In der therapeutischen Wirksamkeit wurde kein Unterschied zwischen 10\%igem und 15\%igem Carbamidperoxid festgestellt (Reinhardt et al. 1993).

In Anwesenheit von Wasser zerfällt 10\%iges Carbamidperoxid in 3,4\% Wasserstoffperoxid $\left(\mathrm{H}_{2} \mathrm{O}_{2}\right)$ und 6,6\% Harnstoff $\left(\mathrm{H}_{2} \mathrm{~N}-\mathrm{CO}-\mathrm{NH}_{2}\right)($ Attin 2002). Der Harnstoff dient lediglich als Car- 
riermolekül und zerfällt über die Zwischenstufe Ammoniak $\left(\mathrm{NH}_{3}\right)$ zu Kohlenstoff, Wasserstoff und Stickstoff.

\subsubsection{Wasserstoffperoxid $\left(\mathrm{H}_{2} \mathrm{O}_{2}\right)$}

Wasserstoffperoxid $\left(\mathrm{H}_{2} \mathrm{O}_{2}\right)$ ist eine Verbindung aus Sauerstoff und Wasserstoff. Die Zerfallsprodukte von Wasserstoffperoxid in Form von reaktiven Radikalen, Hydroxyl- oder Perhydroxylionen stellen die aktive Bleichsubstanz dar (Attin 1998, Christensen 1989, Christensen 1991, Feinman et al. 1991, Goldstein und Garber 1995). Die Zerfallsprodukte spalten ungesättigte $\mathrm{C}=\mathrm{C}$-Doppelbindungen organischer Substanzen auf. Somit beruht ihre Wirkung auf Oxidation verfärbter organischer Komponenten durch den freiwerdenden naszierenden Sauerstoff (Wiegand und Attin 2002).

Chromatogene haben durch ihre hohe Anzahl von Doppelbindungen die Eigenschaft, Licht zu absorbieren und somit farbig zu wirken. Durch die oxidative Spaltung der Doppelbindungen wird das Licht nicht mehr absorbiert, es wird reflektiert oder kann die Zahnhartsubstanz ungehindert durchfluten, wodurch der Eindruck strahlend weißer Zähne entsteht. Des Weiteren wird farbiges Metalloxid wie $\mathrm{Fe}_{2} \mathrm{O}_{3}\left(\mathrm{Fe}^{3+}\right)$ durch Reduktion zu farblosem $\mathrm{FeO}\left(\mathrm{Fe}^{2+}\right)$ (Attin 1998). Zudem können farbige Ringmoleküle aufgespalten werden. Die dabei entstehenden ungesättigten Kohlenstoffketten erscheinen durch das Einfügen von Hydroxylgruppen farblos. Die Entfärbung von durch Tetrazyklin verfärbten Zähnen beruht wahrscheinlich auf der oxidativen Degeneration des Chinonringes, welcher im Tetrazyklin enthalten ist (Attin 1998). Durch Zusatz von Träger- und Verdickungsstoffen, wie zum Beispiel Carbopol ${ }^{\circledR}$ (Carboxylpolymethylen), einem wasserlöslichen Polymer der Polyacrylsäure, kann die Bleichwirkung von Carbamidperoxidgelen verlängert werden. Carbopol ${ }^{\circledR}$ verhindert eine zu rasche Freisetzung des Carbamidperoxids und erhöht somit die Dauer der Abgabe aktiver Peroxide um das Drei- bis Vierfache (Hassink 1995).

\subsubsection{Nebenwirkungen von Bleichmitteln}

Bei externen Bleichtherapien mit Carbamidperoxid kann eine Reihe von Nebenwirkungen am Hart- und Weichgewebe der Mundhöhle auftreten.

Hypersensibilitäten der Pulpa und Irritationen der Gingiva werden als häufigste lokale Beschwerden angegeben (Sulieman 2005). Letzteres ist durch eine unzureichende Gestaltung der Bleichschienen zu erklären (Haywood et al. 1990, Tam 1999). Peroxide können während der Bleichtherapie in Schmelz und Dentin penetrieren. Auch in der Pulpa konnten kleine Mengen von Peroxiden nachgewiesen werden (Cooper et al. 1992, Thitinanthapan et al. 1999). In Abhängigkeit von Konzentration und Einwirkdauer können Peroxide zu Entzün- 
dungsreaktionen der Pulpa führen, die sich auch histologisch nachweisen lassen (Leonard et al. 1997). Dadurch erklären sich die auftretenden Zahnempfindlichkeiten (Haywood und Heymann 1991, Hupfauf und Nolden 1995). Darüber hinaus wird als weitere Nebenwirkung das kurzzeitige Austrocknen der Zähne während der Bleichtherapie registriert. Diese Hypersensibilitäten sind in der Regel temporär und nach Absetzen der Therapie reversibel (Barnes et al. 1998, Leonard et al. 1997). In seltenen Fällen kann es durch das Bleichen zu Wurzeloder internen Resorptionen kommen (Ernst et al. 1995, Trope 1997). Eine Veränderung der Schmelzoberfläche durch Bleichmittel konnte durch mehrere Studien belegt werden (BenAmar et al. 1995, Josey et al. 1996, Haywood et al. 1990). Dabei handelt es sich um eine Reduktion der Mikrohärte durch ein Herauslösen von Kalzium, Phosphat und Fluorid (Burgmaier et al. 2002, Potocnik et al. 2000). Durch Remineralisierungsmaßnahmen mit Fluorid konnten die Porositäten, die im mikroskopischen Bild initialen Kariesläsionen ähneln, stabilisiert werden (Attin 1997, Duschner 2002). Nicht nur die Schmelzoberfläche wird durch Carbamidperoxid beeinflusst, sondern auch bestehende Adhäsivrestaurationen. Der Bleichvorgang führt zu einem Erweichen der Kompomere und die Oberflächenrauigkeit nimmt zu (Attin et al. 2004, Bailey und Swift 1992, Lee et al. 2002). Direkt nach dem Bleichvorgang haben adhäsive Komposite eine reduzierte Haftkraft an der Zahnoberfläche. Aus diesem Grund empfiehlt es sich, erst zwei bis drei Wochen nach Beendigung des Bleichens definitive Versorgungen vorzunehmen (Wiegand und Attin 2002). Amalgamoberflächen sollten vor Beginn der Bleichtherapie mit Kavitätenlack, zum Beispiel Copalite ${ }^{\circledR}$, versiegelt werden, da eine erhöhte Quecksilber-Exposition während der Carbamidperoxidanwendung nachgewiesen wurde (Robertello et al. 1999, Rotstein et al. 2000).

Wasserstoffperoxid ist höchst reaktiv und kann in hohen Dosen zu Schleimhautnekrosen führen. Im Tierversuch verursachte das Verschlucken von größeren Mengen Magenulzera (Attin und Kielbassa 1995, Haywood et al. 1994). Die Bleichgelees, die bei der Therapie am Menschen zum Einsatz kommen, beinhalten aber eine weitaus niedrigere Konzentration von Wasserstoffperoxid. In der Literatur sind bisher keine systemisch toxischen Wirkungen von gebräuchlichen Dosierungen von Carbamidperoxid beschrieben (Feinman 1994, Haywood und Heymann 1991, Woolverton et al. 1993).

\subsection{Wahrnehmung und Bestimmung der Zahnfarbe}

Für das menschliche Auge ist Licht mit einer Wellenlänge von 380 - 780 nm sichtbar (Grehn und Krause 1998). Für die Farbwahrnehmung sind zunächst die in der Netzhaut des Auges liegenden Zäpfchen verantwortlich, welche die Farbinformationen aufnehmen, ehe sie elektrochemisch über die Nerven an das Gehirn weitergeleitet werden. Je nach Wellenlänge (beziehungsweise Energie der Photonen) werden hier die Farbempfindungen von violett über 
blau, grün, gelb bis rot hervorgerufen. Weißes Licht entsteht, wenn alle Wellenlängen zusammentreffen (Grote et al. 2008).

Das Licht vermittelt die Farbe eines Gegenstandes. Das auf eine Oberfläche auftreffende Licht wird je nach dessen Oberflächenbeschaffenheit und Struktur absorbiert, reflektiert und gebrochen. Das resultierende reflektierte Restlicht transportiert so optische Oberflächeninformationen. Wenn es anschließend auf unsere Netzhaut trifft, bestimmt es, welche Farbe wir erkennen. Dabei gibt es allerdings auch eine Reihe von Phänomenen, die die Farbwahrnehmung bis hin zur optischen Täuschung beeinflussen, wie zum Beispiel die Metametrie. Durch besondere physikalisch-chemische Eigenschaften der Pigmente erscheinen dabei zwei Farben in einem bestimmten Licht identisch, bei einer anderen Lichtquelle mit anderem Spektrum wiederum unterschiedlich. In der zahnärztlichen Praxis begegnet man diesem Phänomen bei der Bestimmung der Zahnfarbe unter Tageslicht und/oder unter künstlichem Licht (Reis 1995). Damit ein Zahn natürlich und lebendig erscheint, spielt neben der Farbe auch die Transluzenz eine große Rolle. Sie hängt von der Anzahl an Trübungspartikeln (Streuung des Lichts) im Zahn ab und wird mit dem Begriff der Opazität beschrieben. Darüber hinaus sind vor allem im Dentin Fluoreszenzeigenschaften nachgewiesen worden (Reis 1995).

Die Farbwahrnehmung wird von etlichen weiteren Faktoren beeinflusst, so zum Beispiel der Physiologie des Seeorgans (z.B. Farbenfehlsichtigkeit), der Farbe des Umfeldes (Lippenstift, Kleidung des Patienten), der Dauer der Betrachtung, der Form und der Oberflächengestalt des Objektes und des Betrachtungswinkels (Strub et al. 2005b). Nicht zuletzt hängt der Farbeindruck auch von psychischen und physischen Beeinträchtigungen (Krankheit, Gemütszustand) des Betrachters ab (Strub et al. 2005b).

Die genannten beeinflussenden Faktoren erfordern letztlich ein standardisiertes Verfahren zur guten und verlässlichen und insbesondere reproduzierbaren Farbbestimmung. Dies versuchte man bisher visuell anhand von normierten Farbmustern annähernd zu gewährleisten. Neuerdings werden nun zunehmend auch elektronische Farbmessgeräte eingesetzt, die eine digitale und damit parametrische und besser reproduzierbare Farbbestimmung ermöglichen. Bei der visuellen Methode wird die Zahnfarbe des Patienten mit einem Farbmuster (so genannte Farbringe) abgeglichen (Baumann und Schifferdecker 1994, Musil und Kittler 1983, van der Burgt et al. 1985). Für die visuelle Farbbestimmung mit Farbringen steht eine Vielzahl von der Industrie angebotener Farbsysteme zur Verfügung, zum Beispiel Chromascop ${ }^{\circledR}$ (Ivoclar, Schaan, Lichtenstein), Biodent ${ }^{\circledR}$ (De Trey Dentsply, Dreieich, Germany), Classical ${ }^{\circledR}$ (Vita Zahnfabrik, Bad Säckingen, Germany) oder Vitapan 3D Master (Vita Zahnfabrik, Bad Säckingen, Germany). Dabei erfolgt eine Einteilung der Zahnfarben von A bis D (gelblich, rötlich, bläulich und gräulich) und von 1 (hell) bis 4 (dunkel). Bei einer neueren Farbscala, dem "Vitapan 3D Master" (Vita Zahnfabrik, Bad Säckingen, Germany) wird ein dritter Para- 
meter, die Farbintensität, mitberücksichtigt (Pascher 2004). Diese Methode der Farbbestimmung ist naturgemäß von der subjektiven Wahrnehmung des Betrachters abhängig und daher begrenzt zuverlässig (Culpepper 1970). Elektronische Farbmessgeräte, wie zum Beispiel Shade $\mathrm{Eye}^{\circledR}{ }^{\circledR}$, versuchen diese Fehlerquellen zu minimalisieren und erlauben eine Analyse auch kleinster Farbdifferenzen (Okubo et al. 1998, Setz et al. 1992, Sproull 1973, van der Burgt et al.1990). In verschiedenen Studien liegt die Reproduzierbarkeit dieser instrumentellen Farbanalysen zwischen $83,3 \%$ und 100\% (Okubo et al. 1998). Demgegenüber liegt die exakte Reproduzierbarkeit visueller Farbbestimmung nur bei 46,6\% (Paul et al. 2002). Bei den Geräten für die instrumentelle Farbanalyse handelt es sich um portable Dreibereichfarbmessgeräte oder Spektralfarbmessgeräte. Bei der Dreibereichfarbmessung wird das Objekt mit drei Sensoren vermessen. Hierbei wird das Licht durch Filtergruppen in die Primärfarben (rot, grün und blau) zerlegt. Anhand des reflektierten Lichtes wird dann die Farbe ermittelt. Die Dreibereichfarbmessgeräte zeichnen sich durch einfachen Transport und einfache Handhabung aus (Reis 1995). Bei der Spektralfarbmessung wird das reflektierte Licht in einzelne Wellenlängen von 10-20 nm aufgeteilt und analysiert. Dieses System konnte sich bisher kaum durchsetzen, da es auf große Anlagen angewiesen und dadurch entsprechend teuer ist. Die ermittelte Farbe wird in drei Dimensionen anhand der CIE-Lab*Parameter dargestellt. Diese Parameter wurden von der Commission International d'Eclairage (CIE) entwickelt und 1976 als internationaler Standard (DIN 6174) eingeführt (O'Brien et al. 1989). Alle Farben können in diesem ClE-System in einem dreidimensionalen Koordinatensystem, dem Farbraum, dargestellt werden, wobei die Rot-Grün-Achse mit dem $a^{*}$-Wert, die Gelb-Blau-Achse mit dem b*-Wert und die Helligkeit mit dem L*-Wert assoziiert werden (Abb. 2.2).

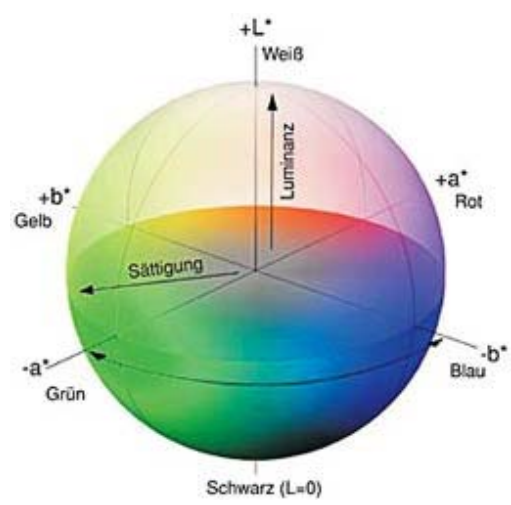

Abbildung 2.2: $\quad$ CIE-Lab* Farbraum

(Quelle:www.haberbeck.de/pages/de/premedia/Colormanagement.html) 
Ein generelles Problem bei der Farbbestimmung mittels instrumenteller Farbanalysegeräte ist jedoch, dass diese für ihre Analyse homogene Strukturen mit glatten Oberflächen benötigen. Da Zähne diesen Anforderungen nur partiell genügen, ist die instrumentelle Farbbestimmung häufig nicht möglich (Haywood et al. 1994). Neben der visuellen und instrumentellen Farbanalyse bietet die photometrische Farbanalyse eine dritte Alternative zur Bestimmung der Zahnfarbe. Bei dieser relativ neuen Methode wird mit Hilfe einer digitalen Spiegelreflexkamera ein Objekt fotografiert und die digitalen Fotos werden anschließend mit Hilfe eines korrespondierenden Computerprogramms, zum Beispiel Adobe Photoshop ${ }^{\circledR}$, farblich ausgewertet (Bengel 2003). Hierbei erhält man, genau wie bei der instrumentellen Farbanalyse, jeweils einen $L^{*}-, a^{*}$ - und $b^{*}$-Wert. Neben der mittlerweile technisch ausgereiften Digitalfotografie und der damit verbundenen Zuverlässigkeit der gewonnenen Farbdaten bietet diese Methode vor allem für wissenschaftliche Arbeiten noch einen wichtigen Vorteil. Die gewonnenen Datensätze der Bilder können durch die Digitalisierung und die komprimierte Archivierung leicht zu einer wiederholten Auswertung oder Statistik herangezogen werden (Bengel 2003, Bentley et al. 1999). Bei wissenschaftlichen Arbeiten im Rahmen von dentalen Farbanalysen stehen Farbveränderungen beziehungsweise Farbvergleiche im Vordergrund. Hierzu sind vergleichende Farbbestimmungsverfahren Voraussetzung, die ein standardisiertes, auch über einen Zeitraum beständiges Aufnahmeverfahren garantieren. Dazu müssen vor allem stets gleiche Licht-, Winkel- und Entfernungseinstellungen gewährleistet sein. Dies ist nur durch eine ortsgebundene Stativfotografie in einer aufbaufesten Apparatur erreichbar. Die bisherig veröffentlichten Daten, die mit Adobe Photoshop ${ }^{\circledR}$ gewonnen wurden, stammten aus klinischen Studien (Knösel et al. 2009, Ziebolz et al. 2007), deren Umfang entsprechend gering war. In der vorliegenden Laborstudie konnten erstmals mit Hilfe des photometrischen Verfahrens größere Datenmengen gewonnen werden, die mit Hilfe einer Statistik signifikant ausgewertet werden konnten. 


\section{Versuchsplan}

Ein Überblick über die Durchführung und den zeitlichen Ablauf der Studie ist der schematischen Darstellung (Abb 3.1) zu entnehmen.

\section{Menschliche Frontzähne}

$\downarrow$

Fixierung eines Stiftes mit quadratischem Querschnitt an den lingualen bzw. palatinalen Flächen der Zähne zur Arretierung in der speziell konstruierten fotografischen Haltevorrichtung

(=> reproduzierbare Position)

Farbbestimmung der Lab*-Werte mit Hilfe von standardisierten digitalen Photos durch das kommerzielle Computerprogramm Adobe Photoshop ${ }^{\circledR}$

$\stackrel{\downarrow}{ } \stackrel{ }{\text { Auslese der Zähne mit } L^{*} \text {-Wert } \geq A 3 \quad\left(L^{*}-\text { Wert=75,4) }\right.}$

$\downarrow$

Einteilung der restlichen 231 Zähne in die drei Versuchsgruppen anhand des

$L^{*}$-Wertes

\begin{tabular}{|c|c|c|}
\hline & & $\downarrow$ \\
\hline $\begin{array}{c}\text { Kontrollgruppe } \\
n=77\end{array}$ & $\begin{array}{c}\text { PF } 15 \% \\
n=77\end{array}$ & $\begin{array}{c}\text { XtraBoost } \\
n=77\end{array}$ \\
\hline
\end{tabular}

$\downarrow \quad \downarrow \quad \downarrow$

\begin{tabular}{|c|c|}
\hline \multicolumn{2}{|c|}{ Bleichphase } \\
\hline $\begin{array}{c}5 \text { mal 8 Stunden mit Opa- } \\
\text { lescence PF 15\% }\end{array}$ & $\begin{array}{c}{ }^{\circledR} \text { mal 15 Minuten mit Opa- } \\
\text { lescence XtraBoost }\end{array}$ \\
\hline
\end{tabular}

$\downarrow \quad \downarrow$

Bestimmung der Lab*-Farben (s.o.)

nach 3 Tagen, 4 Wochen, 12 Wochen und 6 Monaten.

$\downarrow$

statistische Auswertung

Abbildung 3.1: $\quad$ Schematische Darstellung des Versuchsablaufs 


\section{Material und Methode}

\subsection{Material}

\subsubsection{Zahnproben}

Als Prüfkörper wurden 231 menschliche karies- und füllungsfreie Front- und Eckzähne verwendet. Diese wurden 6-18 Monate zuvor in Privatpraxen extrahiert und bis zum Versuchsbeginn in isotoner Kochsalzlösung (B. Braun Melsungen AG, Melsungen/Germany) bei Dunkelheit und Raumtemperatur gelagert.

Im Rahmen der Vorbereitung wurden alle Zähne mit Hilfe von Ultraschall und Polierpaste (Clean Polish, Kerr Hawe, Bioggio/Switzerland) professionell gereinigt. Zähne mit Füllungen und/oder kariösen Läsionen wurden für die Untersuchung nicht berücksichtigt.

\subsubsection{Bleichmittel}

Als Bleichmittel wurden folgende Präparate ausgewählt:

\begin{tabular}{|l|l|l|l|}
\hline Bleichmittel & Zusammensetzung & LOT & Verfahren \\
\hline $\begin{array}{l}\text { Opalescence PF } \\
15 \%\end{array}{ }^{\circledR}$ & $\begin{array}{l}15 \% \text { Carbamidpero- } \\
\text { xid }\end{array}$ & 6512 Lot B2FNR & Home-Bleaching \\
\hline XtraBoost $^{\circledR}$ & $\begin{array}{l}38 \% \text { Wasserstoffpe- } \\
\text { roxid }\end{array}$ & 1301 Lot B2BDR & Chairside \\
\hline
\end{tabular}

Opalescence PF $15 \%{ }^{\circledR}$ und Opalescence XtraBoost ${ }^{\circledR}$ sind Produkte der Firma Ultradent ${ }^{\circledR}$ Produkts, South Jordan, USA. Opalescence PF $15 \%{ }^{\circledR}$ enthält $15 \%$ Carbamidperoxid sowie Kaliumnitrat und Fluorid. Es ist ein Home-Bleaching Präparat und wird mit Hilfe von angepassten Schienen vom Patient selbst auf den Zähnen appliziert. Der Patient muss dazu lediglich zur Beratung und Instruktion, zur Abdrucknahme und zur Anpassung der Schiene (um Irritationen der Gingiva zu vermeiden) die Zahnarztpraxis aufsuchen. Laut Herstellerangaben sollten mit dem Bleichgel 5 -10 Anwendungen durchgeführt werden, wobei in jeder Anwendung eine Einwirkzeit von 2-4 Stunden empfohlen ist. Opalescence XtraBoost $^{\circledR}$ enthält $38 \%$ Wasserstoffperoxid und ist ein Präparat für die In-OfficeZahnaufhellung am Zahnarztstuhl (Chairside-Anwendung). Nachdem die Gingiva durch einen Kofferdam geschützt ist, kann das Bleichgel direkt auf die Zähne aufgetragen werden. Eine Lichtaktivierung ist nicht nötig. Laut Herstellerangaben sollte das Chairside 
Bleichgel für 15 Minuten einwirken. Das Bleichresultat sollte sich nach 2-4 Anwendungen eingestellt haben.

\subsubsection{Versuchsapparatur zur Herstellung standardisierter digitaler Fotos}

Für die standardisierte Versuchsdurchführung wurde durch die klinikeigene Werkstatt eine spezielle Halteapparatur angefertigt, in welche die Kamera und der zu fotografierende Zahn mit einem festen Abstand von $15 \mathrm{~cm}$ zwischen Objektiv und Zahn positioniert werden konnte. Damit wurde eine feste Konstruktion für reproduzierbare Aufnahmen über den gesamten Studienzeitraum geschaffen. Diese gefertigte Apparatur bestand aus einer Schiene mit einem Kamerastativ und einer Halterung für die Zahnproben (Abb. 4.1). An der oralen Fläche eines jeden Zahnes wurde ein 2,5 x 2,5 x 25 mm Metallstift [mit Clearfil Liner Bond $2 \mathrm{~V}^{\circledR}$ (Kuraray Europe $\mathrm{GmbH}$, Düsseldorf/Germany) und LuxaCore ${ }^{\circledR}$ (DMG, Hamburg/Germany)] befestigt. Diese Fixierung diente der einfachen Einsetzung in die Apparatur und gewährleistete zugleich, dass die Zahnproben bei jeder Fotografie immer im gleichen Winkel zum Objektiv standen (Abb 4.2).

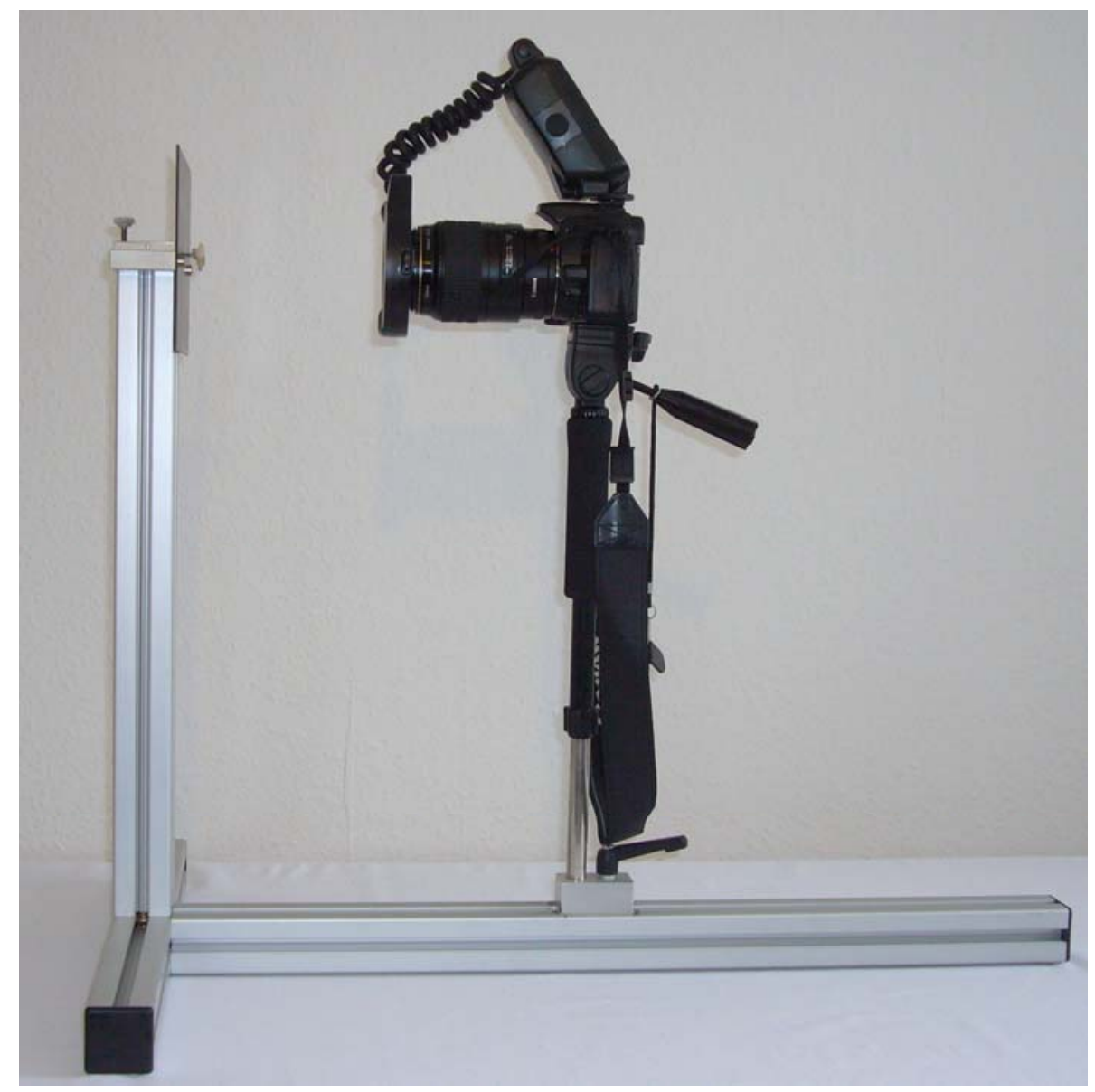

Abbildung 4.1: $\quad$ Haltevorrichtung für Kamera und Zahnprobe 


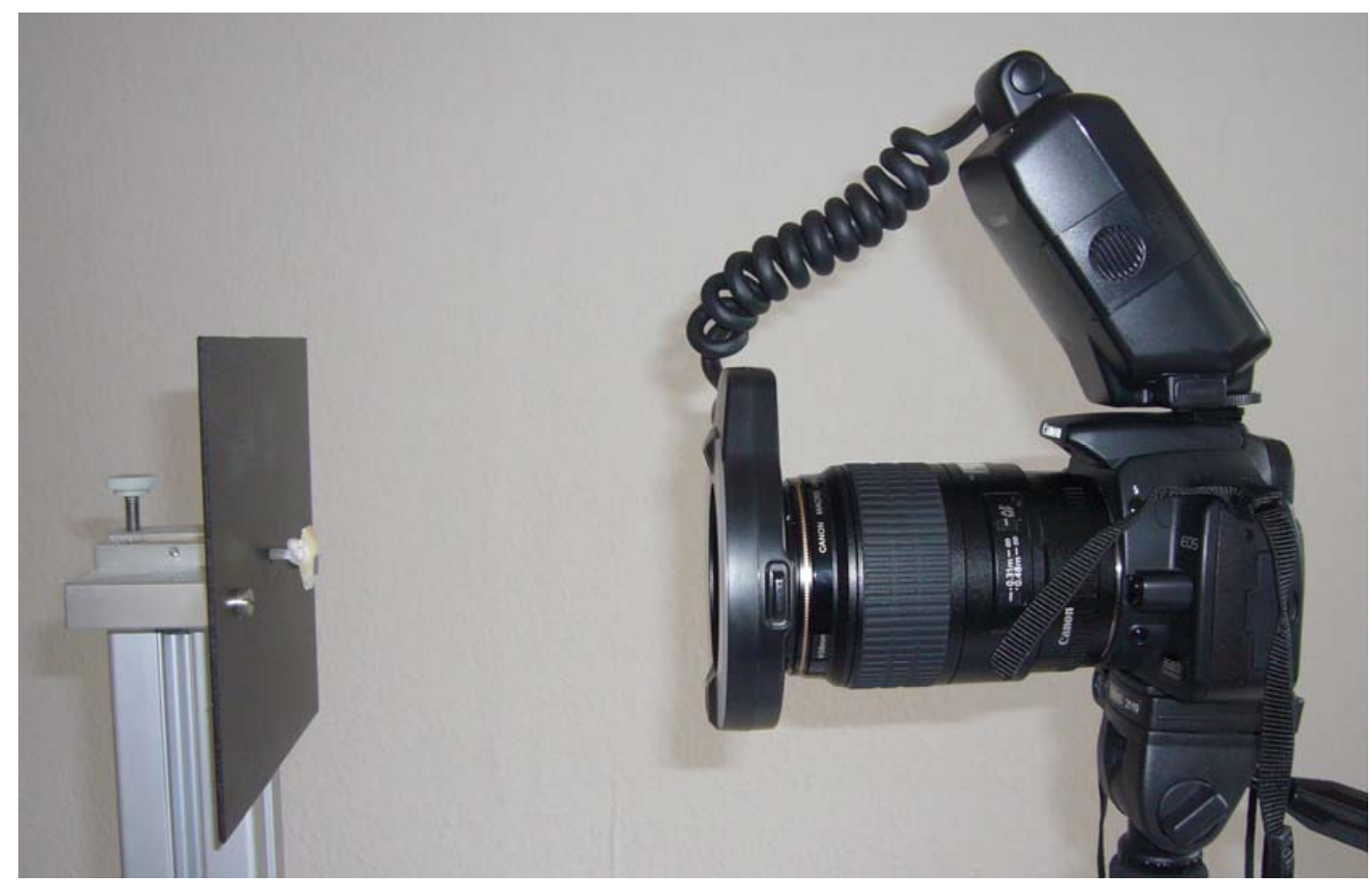

Abbildung 4.2: $\quad$ Kamera und Zahnprobe

\subsubsection{Kamerasystem und Einstellungen}

Für die Erstellung der digitalen Fotos für die fotometrischen Farbbestimmung wurde eine digitale Spiegelreflexkamera vom Typ Canon Eos 350 D (Canon Deutschland GmbH, Krefeld/Germany) verwendet. Die Kamera hatte eine Auflösung von ca. 8,0 Megapixeln, ein Makro-Objektiv, eine E-TTL-II Blitzsteuerung, einen DIGIC-II-Prozessor sowie einen 7Punkt Autofokus. Zudem wurde ein speziell für Nahaufnahmen konzipierter Ringblitz vom Typ Canon MR-14EX (Canon Deutschland $\mathrm{GmbH}$, Krefeld/Germany) verwendet. Dieser setzte direkt an das Makro-Objektiv an und stellte die einzige Lichtquelle während der Fotographie dar. Durch einen komplett abgedunkelten Raum wurde sichergestellt, dass keine anderen Lichtquellen die Farbe oder Helligkeit der Bilder beeinflussen konnten. Der Abstand von Zahn zu Objektiv betrug $15 \mathrm{~cm}$ und alle Fotos wurden mit der Blende 32 bei einer Belichtungszeit von $60 \mathrm{~ms}$ aufgenommen.

\subsection{Methode}

\subsubsection{Farbbestimmung}

Unter den in 4.1.4 beschriebenen Einstellungen wurden standardisierte digitale Zahnfotos erstellt und abgespeichert. Die Farbbestimmung erfolgte anschließend mit Hilfe des 
kommerziell erhältlichen Computerprogramms Adobe Photoshop $\mathrm{CS}^{\circledR}$. Um eine vergleichende Auswertung mit Adobe Photoshop $\mathrm{CS}^{\circledR} \mathrm{zu}$ ermöglichen, ist zunächst ein Grauabgleich notwendig. Hierzu wurde die Graukarte QP Card 101 (QPcardAB, Göteborg, Sweden) verwandt. Diese Graukarte enthält drei Grautöne, dunkles Grau, mittleres Grau (18\%) und Weiß, welche sich im Farbspektrum absolut neutral verhalten. Dadurch war sie ideal verwendbar zur Farbkontrolle und Farbbalancejustierung im Bildverarbeitungsprogramm Adobe Photoshop ${ }^{\circledR}$ (Internet 1). Als Referenzfarbe wurde daher zuvor an jedem Zahn ein Ausschnitt des mittleren Graus (18\%) angebracht (Abb 4.3). Nach dem Grauabgleich wurden mit Hilfe des Bildbearbeitungsprogramms die Lab*Werte des fotografierten Zahnes bestimmt und gespeichert.

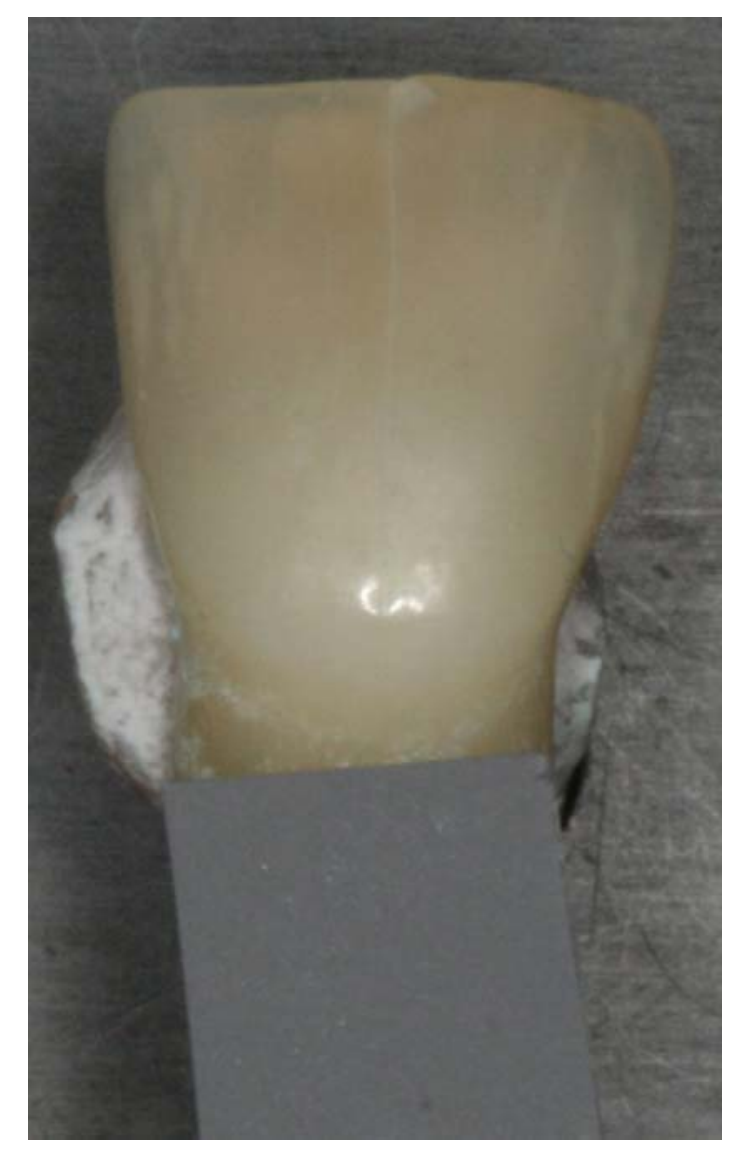

Abbildung 4.3: $\quad$ Zahn mit Graukartenausschnitt

Jedes Bild wurde drei Mal nach gleichem Muster ausgewertet. Aus diesen drei Datensätzen wurde jeweils der Mittelwert gebildet und als definitives Ergebnis zugeordnet. Die Bestimmung der Lab*-Werte in Adobe Photoshop $\mathrm{CS}^{\circledR}$ wurde wie folgt durchgeführt (Bengel 2003): 
1. Unter „Datei öffnen“ entsprechendes Foto wählen.

2. Unter „Filter“ - „Scharfzeichnungsfilter“ - „Unscharf markieren“ auswählen, angezeigtes Viereck direkt in die Mitte des Graupatches bringen, „OK“ drücken.

3. Fenster "Information“ öffnen (falls nicht automatisch offen), dann in diesem Fenster „Navigation/Info/Histogramm“ auf „Info“ stellen.

4. Unter „Bild“ - „Anpassen“ - „Tonwertkorrektur“ mittleres Werkzeug auswählen, auf die Mitte des Graupatches klicken. Dann sollten alle RGB's in der rechten Spalte identisch sein, wenn nicht, den Zeiger minimal hin- und herbewegen und erneut klicken. Wenn alle identisch sind, „OK“ drücken.

5. Unter „Bild“- „Modus“ „Lab-Farbe“ auswählen.

6. Unter „Bild“- „Anpassen“ „Helligkeit/Kontrast“ auswählen.

7. Mit dem Werkzeug wiederum in die Mitte des Graupatches gehen, dort minimal verschieben, bis $a^{*}$ - und $b^{*}$-Wert in der rechten Spalte auf „0“ sind. Dann mit dem Regler die Helligkeit verstellen, bis in der rechten Spalte $L=54$ ist.

8. Unter „Fenster“ „Werkzeuge“ öffnen und mit „Lasso“ entsprechenden Zahnbereich einkreisen.

9. Blitzreflexe mit Pinsel grau abdecken.

10. Im Fenster „Navigation/Info/Histogramm“ auf „Histogramm“ gehen. Programm zeigt für $L^{*}-$, $a^{*}$ - und $b^{*}$-Wert jeweils den Mittelwert und die Standardabweichung an.

Die Lab*-Werte der Auswertung mit Adobe Photoshop $\mathrm{CS}^{\circledR}$ stimmen nicht mit denen der Commission Internationale de L'Eclairage (CIE) überein (Bengel 2003). So reicht die Farbscala des $L^{*}$-Wertes im Computerprogramm Adobe Photoshop $\mathrm{Cs}^{\circledR}$ von 0 bis 255, in der CIE-Scala nur von 0-100. Für eine Umrechnung der Photoshop Lab*-Werte (PS) in Lab*-Werte der CIE (CIE) sind daher spezielle Formeln notwendig (Tab. 4.1).

Tabelle 4.1: Umrechnungsformeln der Lab*-Werte des Computerprogramms Adobe Photoshop CS in Lab*-Werte der CIE nach Bengel (2003).

\begin{tabular}{|l|l|}
\hline Farbwert & \multicolumn{1}{|c|}{ Formel } \\
\hline $\mathrm{L}^{*}$-Wert & $\mathrm{L}(\mathrm{CIE})=\mathrm{L}(\mathrm{PS}) \times 100 / 255$ \\
\hline $\mathrm{a}^{*}$-Wert & $\mathrm{a}(\mathrm{CIE})=(\mathrm{a}(\mathrm{PS})-128) \times 240 / 255$ \\
\hline $\mathrm{b}^{*}$-Wert & $\mathrm{B}(\mathrm{CIE})=(\mathrm{b}(\mathrm{PS})-128) \times 240 / 255$ \\
\hline
\end{tabular}


4.2.2 Randomisierung und Gruppeneinteilung (Kontrollgruppe, PF 15\% und XtraBoost)

Vor Beginn der Studie wurden alle Zähne fotografiert und die Lab*-Werte ermittelt. Anhand der ermittelten $L^{*}$-Werte erfolgte die Zuteilung der Zähne in eine der drei Versuchsgruppen: Gruppe A: PF 15\%, Gruppe B: XtraBoost und Gruppe C: Kontrollgruppe (siehe Kap 4.2.1 Fotografieren und Farbbestimmung der Zähne). Um einen repräsentativen Bleicherfolg zu erzielen, war es notwendig, dass alle Zähne mindestens die Zahnfarbe A3 $\left(=L^{*}\right.$-Wert 75,4$)$ oder dunkler aufwiesen. Daher wurden alle Zähne, die heller als $L^{*}$-Wert 75,4 waren, aussortiert (siehe Tab 4.2).

Tabelle 4.2: Zuordnung der Lab*-Werte zu den ABCD Farben

\begin{tabular}{|c|c|c|c|c|c|c|c|c|c|c|c|c|c|c|c|c|}
\hline & $\mathrm{B} 1$ & $\overline{A 1}$ & B2 & $\overline{\mathrm{D} 2}$ & $\bar{A} 2$ & $\mathrm{C1}$ & $\mathrm{C} 2$ & $\mathrm{D} 4$ & $\overline{A 3}$ & $\overline{B 3}$ & D3 & A3.5 & B4 & $\mathrm{C3}$ & $\mathrm{A} 4$ & $\mathrm{C4}$ \\
\hline No & 1 & 2 & 3 & 4 & 5 & 6 & 7 & 8 & 9 & 10 & 11 & 12 & 13 & 14 & 15 & 16 \\
\hline$L^{*}$ & 78.9 & 79.6 & 76.7 & 75.3 & 76 & 74.2 & 71 & 71.9 & 75.4 & 72.6 & 74.1 & 72.3 & 71.8 & 68.8 & 68.6 & 64.8 \\
\hline$a^{*}$ & -1.76 & -1.61 & -1.62 & -0.54 & -0.08 & -1.26 & -0.22 & -1.03 & 1.36 & 0.47 & 0.62 & 1.48 & 0.5 & -0.01 & 1.58 & 1.59 \\
\hline$b^{*}$ & 12.33 & 13.05 & 16.62 & 13.47 & 16.73 & 12.56 & 16.72 & 17.77 & 19.61 & 22.34 & 16.14 & 21.81 & 22.15 & 16.68 & 21 & 18.66 \\
\hline$\Delta \mathrm{L} *$ & & -0.7 & 2.9 & 1.4 & -0.7 & 1.8 & 3.2 & -0.9 & -3.5 & 2.8 & -1.5 & 1.8 & 0.5 & 3 & 0.2 & 3.8 \\
\hline$\Delta \mathrm{E}$ & & 0.99 & 4.61 & 3.61 & 3.38 & 4.7 & 5.39 & 1.61 & 5.42 & 3.64 & 6.4 & 5.74 & 1.15 & 6.16 & 4.52 & 4.45 \\
\hline
\end{tabular}

Nach dieser Auslese waren noch 231 Zähne vorhanden. Anhand des L*-Wertes wurden die Zähne in 6 Gruppen eingeteilt (Tab 4.3) und anschließend aus diesen heraus zufällig in die drei Versuchsgruppen gelost (Randomisierung).

Tabelle 4.3: L*-Bereichseinteilung der Zähne während der Gruppenzulosung

\begin{tabular}{|l|l|l|}
\hline Gr.-Nr. & L*-Wertbereich & Anzahl der Zähne \\
\hline 1 & $-60,12$ & 6 \\
\hline 2 & $60,13-63,79$ & 27 \\
\hline 3 & $63,80-66,28$ & 39 \\
\hline 4 & $66,29-69,06$ & 48 \\
\hline 5 & $69,07-71,9$ & 72 \\
\hline 6 & $71,91-75,39$ & 39 \\
\hline
\end{tabular}

Zunächst wurden die Zähne der L*-Gruppe "1“ nach dem Zufallsprinzip in die drei Versuchsgruppen Kontrollgruppe, PF15\% oder XtraBoost zugelost. So erhielt jede Gruppe zwei Zähne. Nachdem die sechs Zähne der L*-Wertgruppe „1“ verteilt waren, wurde das- 
selbe Verteilungsverfahren mit den Zähnen der L*-Wertgruppen „2“ bis „6“ durchgeführt. Durch diese Randomisierung wurde eine Gleichverteilung nach $L^{*}$-Werten auf die drei Versuchsgruppen erreicht und jede der drei Gruppen Kontrollgruppe, PF15\% und XtraBoost erhielt die gleiche Anzahl von $n=77$ Zähnen mit dem gleichen durchschnittlichen Ausgangs $L^{*}$-Wert von $68,24 \pm 0,8$.

\subsubsection{Lagerung}

Während der vorbereitenden Maßnahmen und im weiteren Versuchsverlauf lagerten die Zähne in künstlicher Speichellösung, die alle 7 Tage erneuert wurde. Der künstliche Speichel enthielt folgenden Bestandteilen in entsprechenden Mengen (Tab 4.4):

Tabelle 4.4: Zusammensetzung des künstlichen Speichels nach Klimek et al. $(1982,157)$

\begin{tabular}{|l|l|}
\hline Bestandteil & Mengenangabe \\
\hline Ascorbinsäure & $0,002 \mathrm{~g}$ \\
\hline Glukose & $0,030 \mathrm{~g}$ \\
\hline $\mathrm{NaCl}$ & $0,580 \mathrm{~g}$ \\
\hline $\mathrm{CaCl}_{2}$ & $0,170 \mathrm{~g}$ \\
\hline $\mathrm{NH}_{4} \mathrm{Cl}$ & $0,160 \mathrm{~g}$ \\
\hline $\mathrm{KCl}$ & $1,270 \mathrm{~g}$ \\
\hline $\mathrm{NaSCN}$ & $0,160 \mathrm{~g}$ \\
\hline $\mathrm{KH} 2 \mathrm{PO} 4$ & $0,330 \mathrm{~g}$ \\
\hline Harnstoff & $0,200 \mathrm{~g}$ \\
\hline Muzin & $2,700 \mathrm{~g}$ \\
\hline Aqua dest. & $1000 \mathrm{ml}$ \\
\hline
\end{tabular}

Vor Versuchsbeginn wurden die Zahnproben bei Dunkelheit und Raumtemperatur aufbewahrt. Während des Versuchsverlaufes lagerten die Proben abwechselnd für 2 Wochen in einem Klimaschrank bei $37^{\circ} \mathrm{C}$ und völliger Dunkelheit und für 2 Wochen bei Tageslicht und Raumtemperatur. 


\subsubsection{Bleichvorgang}

Ziel der Bleichtherapie war es, ein nahezu gleiches Bleichergebnis (gleicher Ausgangswert) bei beiden Bleichverfahren zu erzielen. Um weiterhin ein gute Vergleichbarkeit in der Langzeitwirkung der beiden Verfahren zu erhalten, war es zusätzlich nötig, beide Versuchsgruppen einer deutlichen Bleichwirkung zu unterziehen. Um dieses Bleichergebnis für beide Gruppen zu erreichen, war es erforderlich, den Bleichvorgang zu modifizieren und nicht nach Herstellerangaben zu bleichen. In Anlehnung an eine Studie von Auschill und Hellwig (2005) erfolgte die Bleichtherapie daher in modifizierter Form (Tab 4.5).

Tabelle 4.5: Bleichintervalle der Gruppen mit aktiver Bleichsubstanz.

\begin{tabular}{|l|l|l|}
\hline $\begin{array}{l}\text { (Produkt- und) } \\
\text { Gruppenname }\end{array}$ & $\begin{array}{l}\text { Aktive } \\
\text { Bleichsubstanz }\end{array}$ & Bleichintervall \\
\hline Kontrollgruppe & -------- & -------- \\
\hline $\begin{array}{l}\text { PF 15\% } \\
\text { Opalescence) }\end{array}$ & $\begin{array}{l}\text { Carbamidperoxid } \\
\text { mit Frasaco Strip } \\
\text { Kronen }\end{array}$ \\
\hline $\begin{array}{l}\text { (Opalescence) } \\
\text { XtraBoost }\end{array}$ & $\begin{array}{l}38 \% \\
\text { Wasserstoffperoxid }\end{array}$ & 3 mal 15 Minuten \\
\hline
\end{tabular}

Um die äußeren Einflüsse der Studie und die natürlichen Veränderungen der Zahnfarbe über den Studienverlauf erfassen zu können, wurde die Kontrollgruppe nicht aufgehellt. Da das Home-Bleaching Verfahren in der Regel mit Hilfe von individuell angefertigten Kunststoffschienen durchgeführt wird, war es sinnvoll auch dies während des Bleichvorgangs nachzustellen. Unter Zuhilfenahme von Frasaco Stripkronen ${ }^{\circledR}$ (Frasaco GmbH, Tettnang, Germany) war es möglich dieses Home-Bleaching Verfahren zu imitieren. Das Bleichmittel Opalescence PF $15 \%{ }^{\circledR}$ (Ultradent, South Jordan, USA) wurde in die Frasaco Stripkronen ${ }^{\circledR}$ appliziert, diese anschließend über den Zahn gestülpt und in künstliche Speichellösung gelegt (Abb. 4.4). Somit wurden die Bleichbedingungen beim HomeBleaching Verfahren bestmöglich und praxisnah nachgeahmt. Dieses Verfahren wurde an 
fünf aufeinander folgenden Tagen für jeweils acht Stunden wiederholt, somit lagen zwischen den Bleichintervallen 16 Stunden.

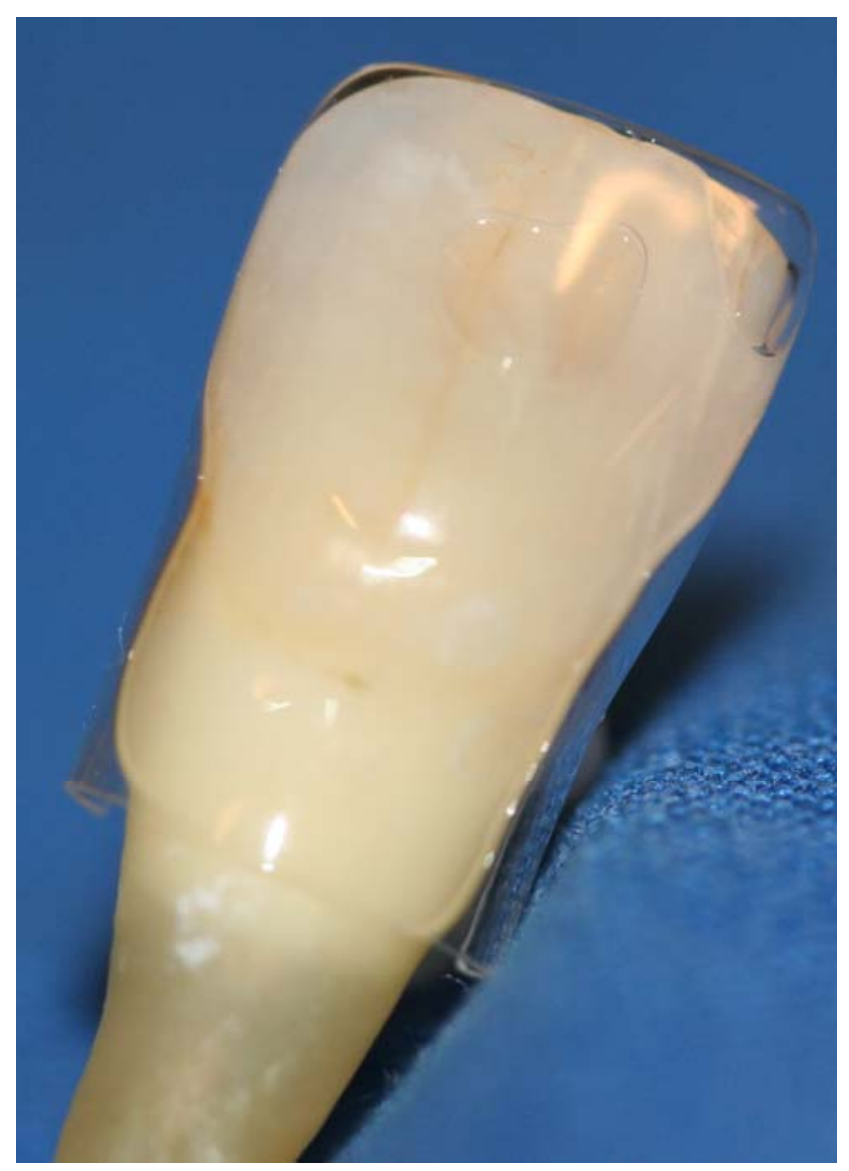

Abbildung 4.4: $\quad$ Zahn mit Frasaco Stripkrone ${ }^{\circledR}$

Die Gruppe XtraBoost wurde mit einem 38\%igen Wasserstoffperoxid-Bleichmittel von Opalescence ${ }^{\circledR}$ behandelt. Da bei In-Office-Verfahren eine absolute Trockenlegung unter Kofferdam vom Hersteller vorgeschrieben wird, wurden die Zähne vor dem Bleichvorgang getrocknet. Anschließend wurden sie mit dem Bleichmittel Opalescence XtraBoost ${ }^{\circledR}$ (Ultradent, South Jordan, USA) bestrichen, welches außerhalb der Speichellösung 15 Minuten einwirken konnte. Dieses Verfahren wurde an drei aufeinander folgenden Tagen wiederholt, somit lagen zwischen den Bleichintervallen 24 Stunden. Auf eine Lichtaktivierung mit UV-Licht wurde verzichtet.

\subsubsection{Farbbestimmung während des Studienverlaufs}

Da Carbamit-Peroxid-Präparate Zähnen Wasser entziehen, wurden die extrahierten Zähne erst drei Tage nach dem Bleichvorgang fotografiert. Somit sollte eine Farbveränderung der Zähne aufgrund von Wasserentzug ausgeschlossen werden. 
Weitere Aufnahmen erfolgten nach 4, 12 und 24 Wochen. In der Zeit zwischen den Aufnahmen lagerten die Zähne in künstlichem Speichel (Kap 4.2.3). 10 Minuten vor dem Fotografieren wurden die Proben aus der Speichellösung herausgenommen und mit einem Zellstofftuch getrocknet, um unnötige Reflexe aufgrund von Flüssigkeit auf der Zahnoberfläche zu vermeiden.

Direkt nach der Aufnahme wurden die Zähne wieder in die künstliche Speichellösung zurückgelegt, um ein Austrocknen der Zahnproben zu vermeiden.

\subsection{Statistische Auswertung}

Die abschließende Auswertung erfolgte mit Hilfe des statistischen Auswertungsprogramms SAS $9.1^{\circledR}$ (Statistical Analysis System, Institute, Cary, NC, USA). Die Daten wurden mit Hilfe von repeated measurements (ANOVA), einer Varianzanlayse mit wiederholten Messungen, ausgewertet. Das Signifikanz-Niveau wurde dabei auf $p \leq 0,05$ festgelegt. Das Ergebnis dieser statistischen Tests ist ein so genannter p-Wert, der systemische Effekte von zufälligen Erscheinungen abgrenzt und somit die stochastische Irrtumswahrscheinlichkeit widerspiegelt. Liegt dieser p-Wert unterhalb des gewählten Signifikanz-Niveaus (zum Beispiel $p=0,01$ ), so wird der Unterschied als signifikant bezeichnet, das heißt, dass dieser Unterschied mit einer bestimmten Wahrscheinlichkeit nicht durch Zufall zu Stande gekommen ist.

Die statistische Auswertung erfolgte in Zusammenarbeit mit der Abteilung für Medizinische Statistik der Universitätsmedizin Göttingen (Direktor: Prof. Dr. E. Brunner). 


\section{$5 \quad$ Ergebnisse}

\subsection{Veränderung: Baseline zu t1}

$L^{*}$-Wert

Die Bleichtherapie führt in beiden Gruppen PF15\% und XtraBoost zu einer signifikanten Änderung des $L^{*}$-Wertes $(p<0,0001)$ und somit zu einer deutlichen Aufhellung der behandelten Zähne. In der Kontrollgruppe, die nicht mit Bleichgel behandelt wurde, war keine Farbveränderung feststellbar ( $p=0,0531)$ (Abb 5.1, Tab 5.1).

Tabelle 5.1: Veränderung L*-Wert Baseline zu t1

\begin{tabular}{|l|l|l|l|l|}
\hline $\mathrm{L}^{*}$ & $\begin{array}{l}\text { Mittelwert } \\
\text { Baseline } \\
\mathrm{L} \pm \mathrm{s}\end{array}$ & $\begin{array}{l}\text { Mittelwert } \\
\mathrm{T} 1 \mathrm{~L} \pm \mathrm{s}\end{array}$ & $\Delta \mathrm{L} \pm \mathrm{s}$ & $\begin{array}{l}\text { Signifikanzniveau } \\
\mathrm{K}\end{array}$ \\
\hline Kontrollgruppe & $68,16 \pm 3,84$ & $69,69 \pm 3,6$ & $1,53 \pm 3,45$ & 0,0531 \\
\hline PF 15\% & $68,23 \pm 3,79$ & $72,48 \pm 3,87$ & $4,33 \pm 4,99$ & $<0,0001$ \\
\hline Xtra Boost 38\% & $68,32 \pm 3,7$ & $73,25 \pm 3,35$ & $4,88 \pm 4,62$ & $<0,0001$ \\
\hline
\end{tabular}

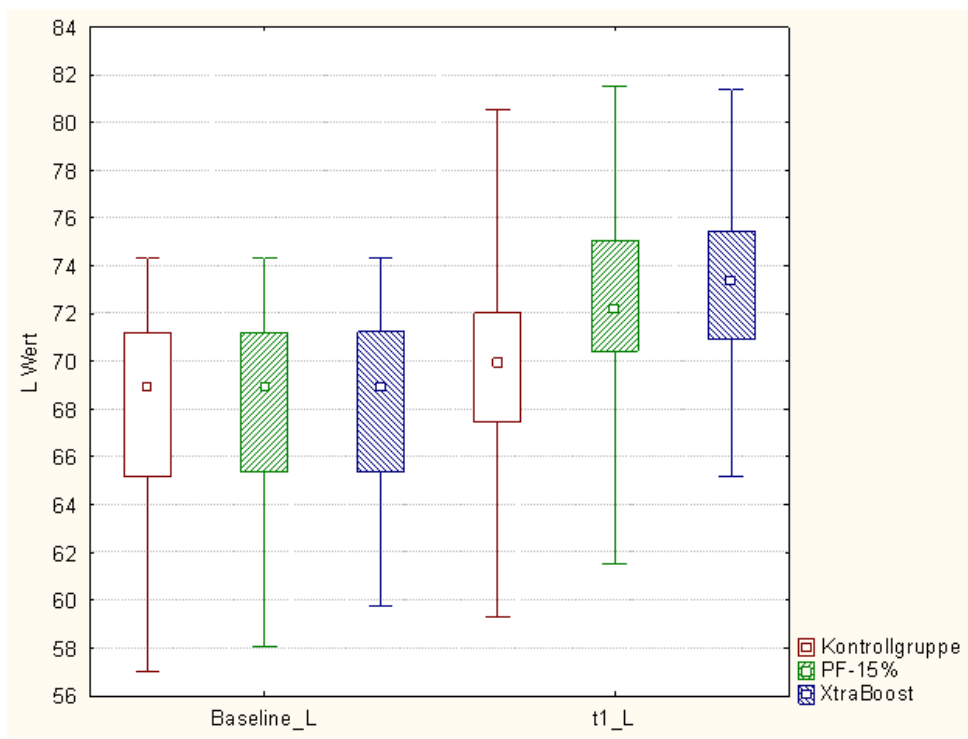

Abbildung 5.1:Veränderung des $L^{*}$-Wertes Baseline zu t1 
$a^{*}$-Wert

Die Bleichtherapie führt in den Gruppen PF15\% ( $p=0,0108)$ und XtraBoost $(p=0,0481)$ zu einer signifikanten Änderung des $a^{*}$-Wertes und somit zu einem feststellbaren Rückgang der Rötlichkeit der extrahierten Zähne. In der nicht behandelten Kontrollgruppe wurde keine Änderung festgestellt ( $p=0,3155)$ (Abb 5.2, Tab 5.2).

Tabelle 5.2: Veränderung $a^{*}$-Wert Baseline zu t1

\begin{tabular}{|l|l|l|l|l|}
\hline $\mathrm{a}^{*}$ & $\begin{array}{l}\text { Mittelwert } \\
\text { Baseline } \\
\mathrm{a} \pm \mathrm{s}\end{array}$ & $\begin{array}{l}\text { Mittelwert } \\
\text { T1 a } \pm \mathrm{s}\end{array}$ & $\Delta \mathrm{a} \pm \mathrm{s}$ & $\mathrm{P}$ \\
\hline Kontrollgruppe & $2,45 \pm 1,50$ & $2,29 \pm 1,61$ & $0,48 \pm 0,80$ & 0,3155 \\
\hline PF 15\% & $2,65 \pm 1,85$ & $1,84 \pm 1,61$ & $0,81 \pm 1,2$ & 0,0108 \\
\hline XtraBoost $38 \%$ & $2,55 \pm 1,65$ & $1,87 \pm 1,87$ & $0,69 \pm 1,16$ & 0,0481 \\
\hline
\end{tabular}

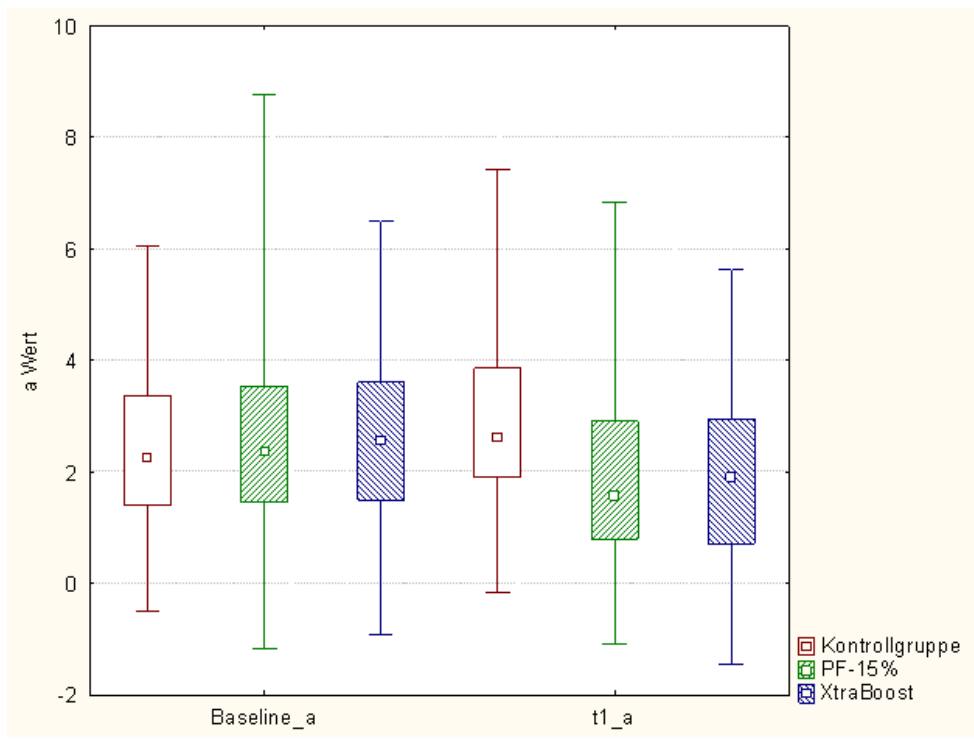

Abbildung 5.2: Veränderung des $a^{*}$-Wertes Baseline zu t1 
$b^{*}$-Wert

Die Bleichtherapie führt nur in der Gruppe PF15\% zu einer signifikanten Änderung des b*Wertes $(p=0,0015)$ und somit zu einer feststellbaren Abnahme der Gelblichkeit der behandelten Zähne. In der unbehandelten Kontrollgruppe $(p=0,3603)$ und der XtraBoost-Gruppe $(p=0,0623)$ war keine Gelbaufhellung feststellbar (Abb 5.3, Tab 5.3).

Tabelle 5.3: Veränderung $b^{*}$-Wert Baseline zu t1

\begin{tabular}{|l|l|l|l|l|}
\hline $\mathrm{B}^{*}$ & $\begin{array}{l}\text { Mittelwert } \\
\text { Baseline } \\
\mathrm{b} \pm \mathrm{s}\end{array}$ & $\begin{array}{l}\text { Mittelwert } \\
\mathrm{T} 1 \mathrm{~b} \pm \mathrm{s}\end{array}$ & $\Delta \mathrm{b} \pm \mathrm{s}$ & $\mathrm{P}$ \\
\hline Kontrollgruppe & $16,14 \pm 3,13$ & $15,31 \pm 2,59$ & $0,83 \pm 1,98$ & 0,3603 \\
\hline PF 15\% & $16,59 \pm 2,99$ & $15,03 \pm 2,55$ & $1,56 \pm 1,97$ & 0,0015 \\
\hline XtraBoost 38\% & $16,47 \pm 3,07$ & $15,42 \pm 2,21$ & $1,06 \pm 2,43$ & 0,0623 \\
\hline
\end{tabular}

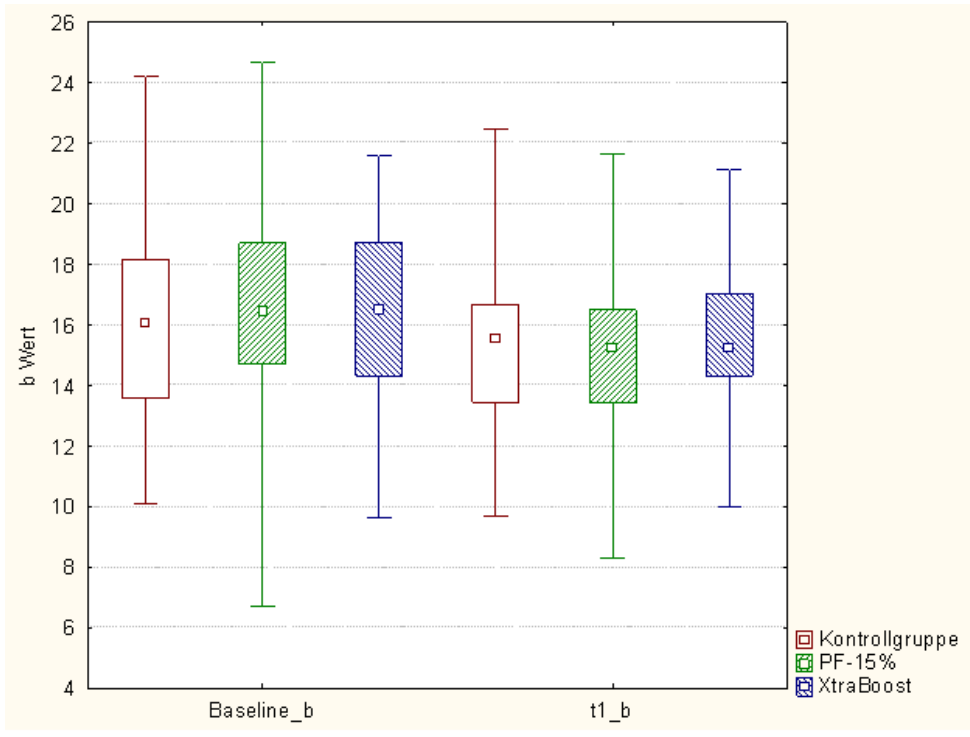

Abbildung 5.3: Veränderung des $b^{*}$-Wertes Baseline zu t1 


\subsection{Veränderung: t1 zu t2}

$L^{*}$-Wert

Im Zeitintervall $\mathrm{t} 1$ bis $\mathrm{t} 2$ kommt es zu einer signifikanten Farbveränderung des $\mathrm{L}^{*}$-Werts nur in der Gruppe PF15\% ( $p=0,0246)$, das heißt, die Zähne wurden durch Nachbleicheffekte weiter aufgehellt. Bei der Gruppe XtraBoost ist dies nicht der Fall $(p=0,0741)$. Auch die Kontrollgruppe bleibt unverändert ( $p=0,9995)$ (Abb 5.4, Tab 5.4).

Tabelle 5.4: Veränderung $L^{*}$-Wert t1 zu t2

\begin{tabular}{|l|l|l|l|l|}
\hline $\mathrm{L}^{*}$ & $\begin{array}{l}\text { Mittelwert } \\
\mathrm{T} 1 \\
\mathrm{~L} \pm \mathrm{s}\end{array}$ & $\begin{array}{l}\text { Mittelwert } \\
\mathrm{T} 2 \\
\mathrm{~L} \pm \mathrm{s}\end{array}$ & $\Delta \mathrm{L} \pm \mathrm{s}$ & $\begin{array}{l}\text { Signifikanzniveau } \\
\mathrm{P}\end{array}$ \\
\hline Kontrollgruppe & $69,69 \pm 3,6$ & $69,81 \pm 3,58$ & $1,12 \pm 2,93$ & 0,9995 \\
\hline PF 15\% & $72,48 \pm 3,87$ & $74,16 \pm 3,4$ & $1,73 \pm 4,99$ & 0,0246 \\
\hline Xtra Boost 38\% & $73,25 \pm 3,35$ & $71,88 \pm 3,19$ & $0,43 \pm 4,30$ & 0,0741 \\
\hline
\end{tabular}

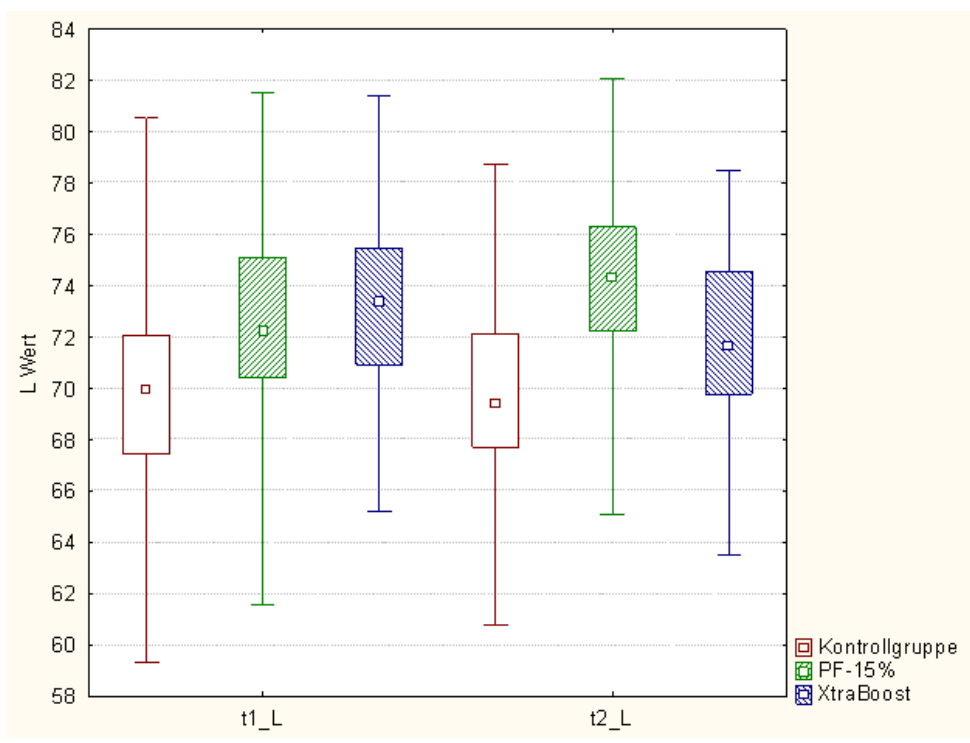

Abbildung 5.4: Veränderung des $L^{*}$-Wertes $\mathrm{t} 1 \mathrm{zu}$ t2 
$a^{*}$-Wert

Im Zeitintervall t1 zu t2 kommt es zu keiner signifikanten Farbveränderung des $a^{*}$-Werts in allen drei Gruppen ( $p>0,05)$ (Abb 5.5, Tab 5.5).

Tabelle 5.5: Veränderung $\mathrm{a}^{*}$-Wert t1 zu t2

\begin{tabular}{|l|l|l|l|l|}
\hline $\mathrm{a}^{*}$ & $\begin{array}{l}\text { Mittelwert } \\
\mathrm{T} 1 \\
\mathrm{a} \pm \mathrm{s}\end{array}$ & $\begin{array}{l}\text { Mittelwert } \\
\mathrm{T} 2 \\
\mathrm{~A} \pm \mathrm{s}\end{array}$ & $\Delta \mathrm{a} \pm \mathrm{s}$ & $\mathrm{P}$ \\
\hline Kontrollgruppe & $2,29 \pm 1,61$ & $3,07 \pm 1,74$ & $0,15 \pm 0,94$ & 0,9773 \\
\hline PF15\% & $1,84 \pm 1,61$ & $1,48 \pm 1,50$ & $0,36 \pm 0,90$ & 0,5928 \\
\hline XtraBoost 38\% & $1,87 \pm 1,87$ & $1,52 \pm 1,71$ & $0,34 \pm 1,46$ & 0,6428 \\
\hline
\end{tabular}

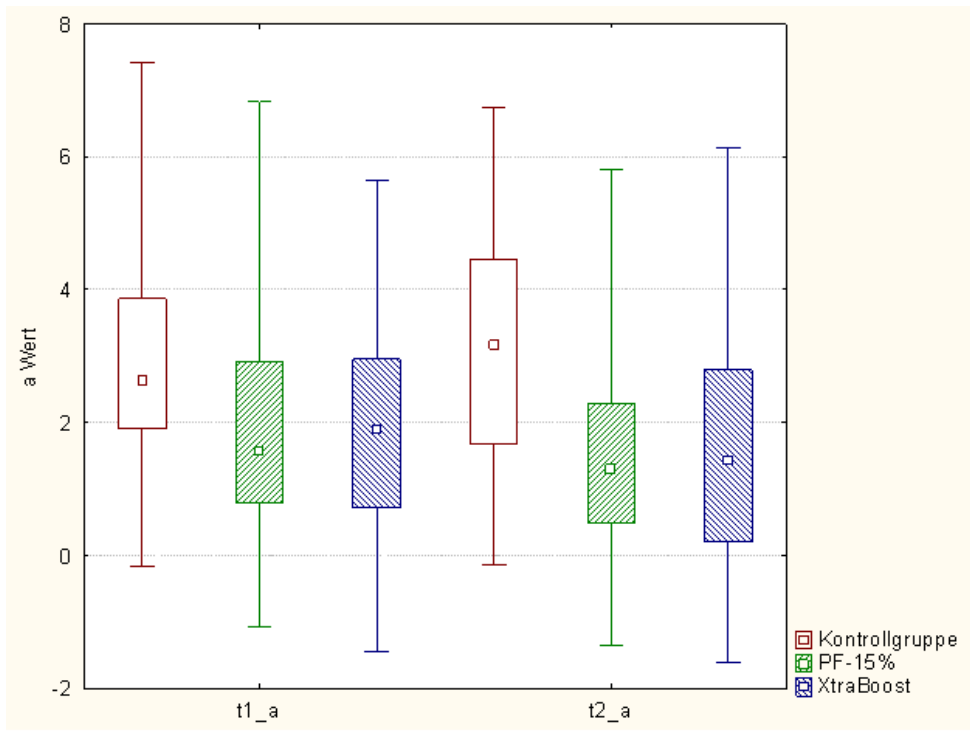

Abbildung 5.5: Veränderung des $a^{*}$-Wertes $t 1$ zu t2 
$b^{*}$-Wert

Im Zeitintervall t1 zu t2 kommt es erneut zu einer signifikanten Farbveränderung in der PF15\%-Gruppe ( $p<0,0001)$, das heißt, die Gelblichkeit der Zähne verändert sich zu helleren Werten hin. Die XtraBoost-Gruppe $(p=0,9922)$ und die Kontrollgruppe $(p=0,3636)$ bleiben unverändert (Abb 5.6, Tab 5.6).

Tabelle 5.6: Veränderung $b^{*}$-Wert t1 zu t2

\begin{tabular}{|l|l|l|l|l|}
\hline $\mathrm{b}^{*}$ & $\begin{array}{l}\text { Mittelwert } \\
\mathrm{T} 1 \\
\mathrm{~b} \pm \mathrm{s}\end{array}$ & $\begin{array}{l}\text { Mittelwert } \\
\text { T2 } \\
\mathrm{B} \pm \mathrm{s}\end{array}$ & $\Delta \mathrm{b} \pm \mathrm{s}$ & $\mathrm{P}$ \\
\hline Kontrollgruppe & $15,31 \pm 2,59$ & $16,13 \pm 2,95$ & $0,82 \pm 1,54$ & 0,3636 \\
\hline PF 15\% & $15,03 \pm 2,55$ & $13,07 \pm 2,51$ & $1,96 \pm 2,13$ & $<0,0001$ \\
\hline XtraBoost 38\% & $15,42 \pm 2,21$ & $15,24 \pm 2,29$ & $0,17 \pm 2,18$ & 0,9922 \\
\hline
\end{tabular}

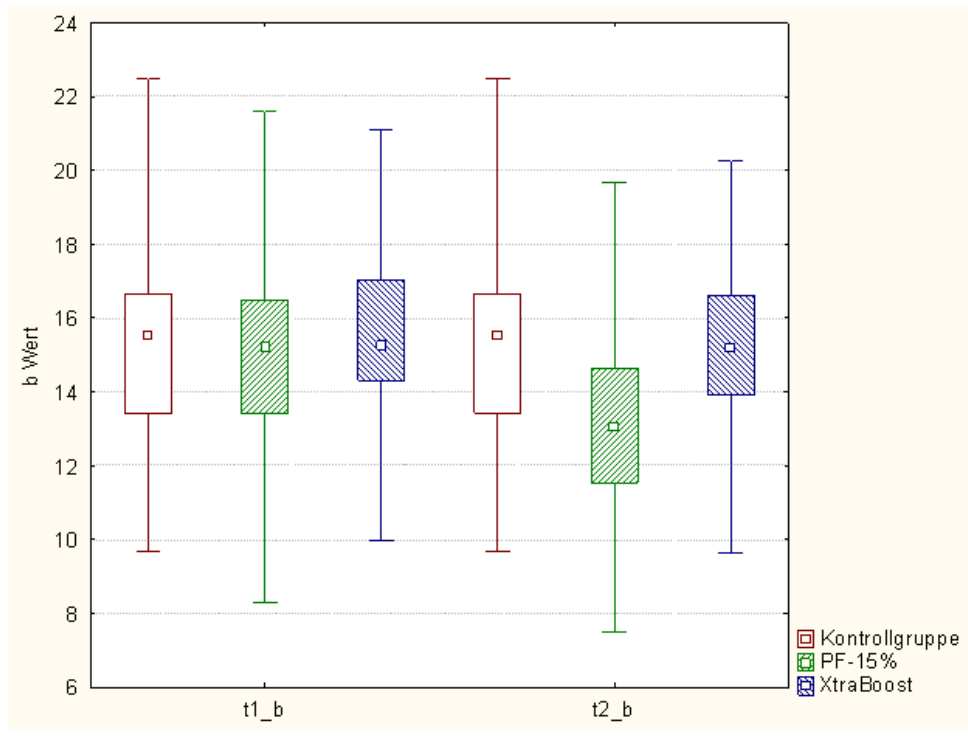

Abbildung 5.6: Veränderung des $b^{*}$-Wertes $\mathrm{t} 1 \mathrm{zu}$ t2 


\subsection{Veränderung: t2 zu t3}

$L^{*}$-Wert

Es konnten keine statistisch feststellbaren Farbveränderungen in der PF15\% und in der XtraBoost-Gruppe zwischen dem Zeitintervallen $\mathrm{t} 2$ und $\mathrm{t} 3$ festgestellt werden (Abb 5.7, Tab $5.7)$.

Tabelle 5.7: Veränderung $L^{*}$-Wert $\mathrm{t} 2$ zu t3

\begin{tabular}{|l|l|l|l|l|}
\hline $\mathrm{L}^{*}$ & $\begin{array}{l}\text { Mittelwert } \\
\mathrm{T} 2 \\
\mathrm{~L} \pm \mathrm{s}\end{array}$ & $\begin{array}{l}\text { Mittelwert } \\
\mathrm{T} 3 \\
\mathrm{~L} \pm \mathrm{s}\end{array}$ & $\Delta \mathrm{L} \pm \mathrm{s}$ & $\begin{array}{l}\text { Signifikanzniveau } \\
\mathrm{P}\end{array}$ \\
\hline Kontrollgruppe & $69,81 \pm 3,58$ & $70,15 \pm 3,27$ & $0,34 \pm 2,66$ & 0,9735 \\
\hline PF 15\% & $74,16 \pm 3,4$ & $73,05 \pm 3,27$ & $1,12 \pm 2,81$ & 0,2712 \\
\hline Xtra Boost 38\% & $71,88 \pm 3,19$ & $72,10 \pm 3,33$ & $0,22 \pm 3,03$ & 0,9933 \\
\hline
\end{tabular}

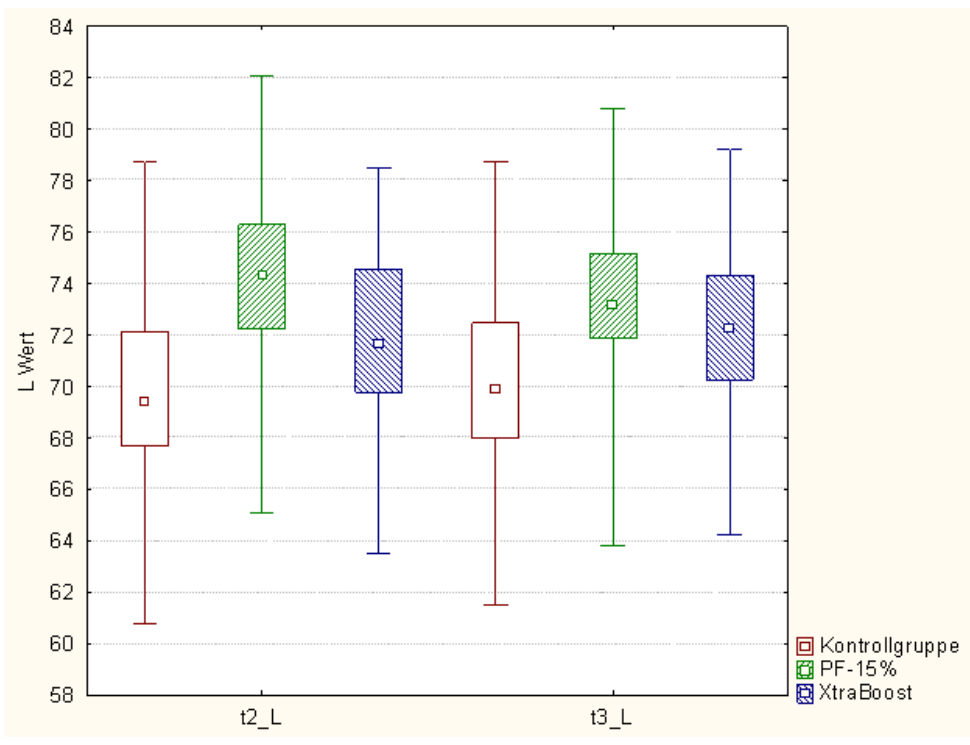

Abbildung 5.7: Veränderung des $L^{*}$-Wertes t2 zu t3 
$a^{*}$-Wert

Im Zeitintervall t2 zu t3 kommt es zu keiner signifikanten Farbveränderung des $a^{*}$-Werts in allen drei Gruppen (Abb 5.8, Tab 5.8).

Tabelle 5.8: Veränderung $a^{*}$-Wert $\mathrm{t} 2$ zu t3

\begin{tabular}{|c|c|c|c|c|}
\hline$a^{*}$ & $\begin{array}{l}\text { Mittelwert } \\
\mathrm{T} 2 \\
\mathrm{a} \pm \mathrm{s} \\
\end{array}$ & $\begin{array}{l}\text { Mittelwert } \\
\text { T3 } \\
\mathrm{A} \pm \mathrm{s}\end{array}$ & $\Delta \mathrm{a} \pm \mathrm{s}$ & $p$ \\
\hline Kontrollgruppe & $3,07 \pm 1,74$ & $2,96 \pm 1,68$ & $0,11 \pm 0,81$ & 0,9922 \\
\hline PF15 \% & $1,48 \pm 1,50$ & $1,28 \pm 1,42$ & $0,19 \pm 0,75$ & 0,9367 \\
\hline XtraBoost 38\% & $1,52 \pm 1,71$ & $1,13 \pm 1,52$ & $0,39 \pm 1,06$ & 0,5235 \\
\hline
\end{tabular}

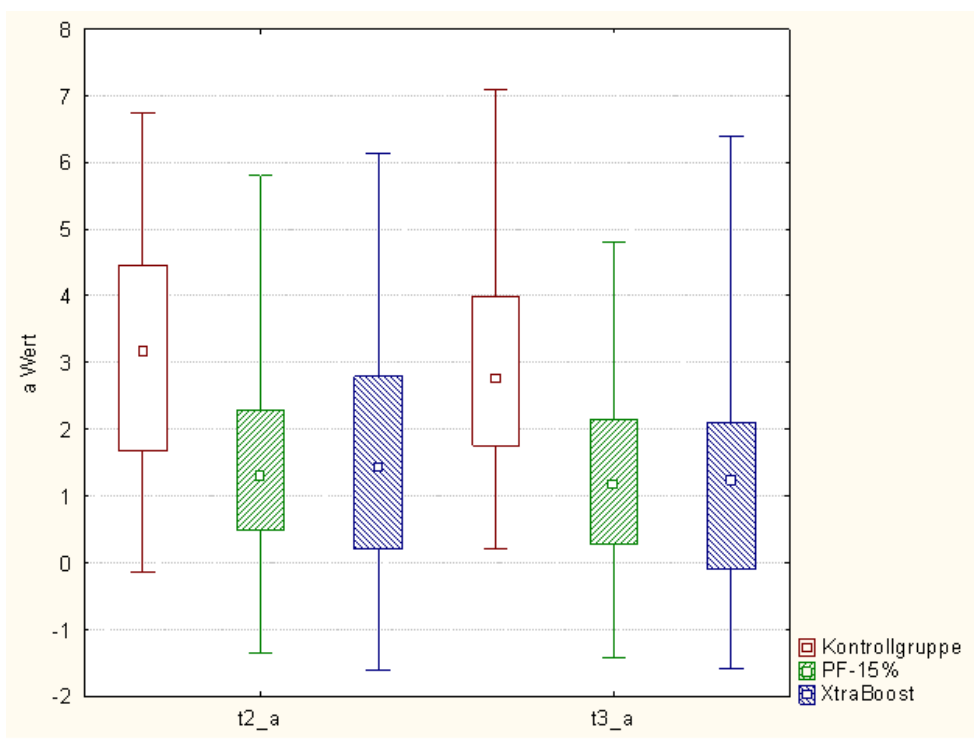

Abbildung 5.8: Veränderung des $a^{*}$-Wertes t2 zu t3 
$b^{*}$-Wert

Im Zeitintervall t2 zu t3 kommt es in keiner Gruppe zu einer statistisch nachweisbaren Veränderung der b*-Werte (Abb 5.9, Tab 5.9).

Tabelle 5.9: Veränderung $b^{*}$-Wert $t 2$ zu t3

\begin{tabular}{|l|l|l|l|l|}
\hline $\mathrm{b}^{*}$ & $\begin{array}{l}\text { Mittelwert } \\
\text { T2 } \\
\mathrm{b} \pm \mathrm{s}\end{array}$ & $\begin{array}{l}\text { Mittelwert } \\
\text { T3 } \\
\mathrm{B} \pm \mathrm{s}\end{array}$ & $\Delta \mathrm{b} \pm \mathrm{s}$ & $\mathrm{P}$ \\
\hline Kontrollgruppe & $\begin{array}{l}16,13 \pm 2,95 \\
15,85 \pm 2,80\end{array}$ & $0,28 \pm 1,71$ & 0,9722 \\
\hline PF 15\% & $13,07 \quad \pm \quad 2,51$ & $13,37 \quad \pm \quad 2,48$ & $0,30 \pm 1,79$ & 0,9479 \\
\hline XtraBoost 38\% & $15,24 \quad \pm \quad 2,29$ & $14,87 \pm 2,58$ & $0,37 \pm 2,06$ & 0,8856 \\
\hline
\end{tabular}

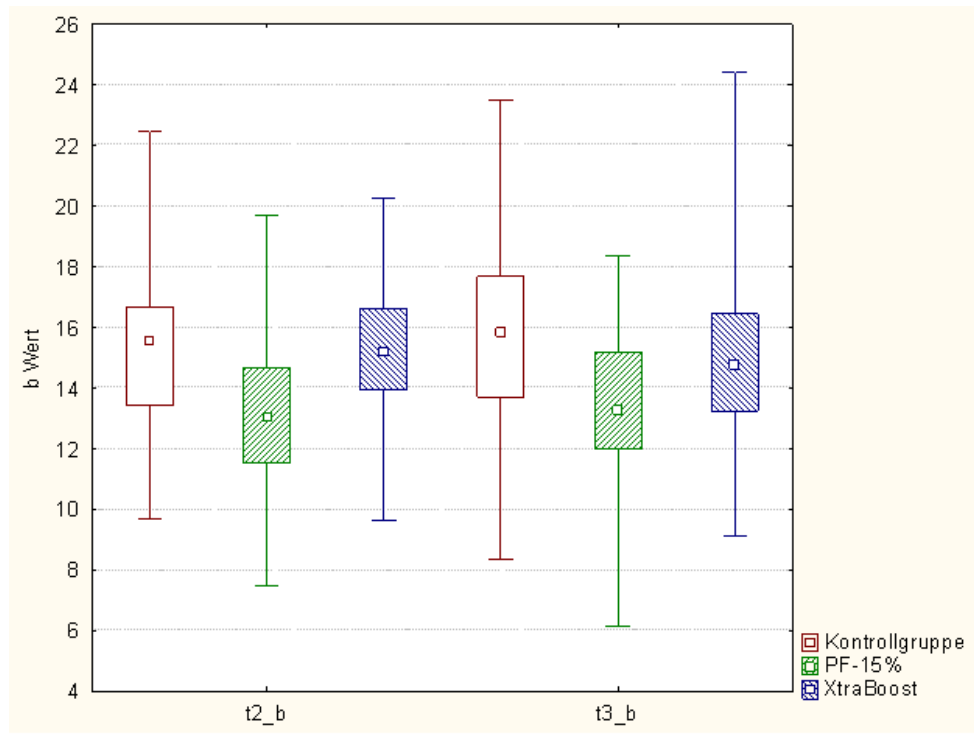

Abbildung 5.9: Veränderung des $b^{*}$-Wertes $t 2$ zu $t 3$ 


\subsection{Veränderung: $\mathrm{t} 3 \mathrm{zu} \mathrm{t} 4$}

$L^{*}$-Wert

Im letzten Zeitintervall, t3 zu t4, kommt es zu einer signifikanten Farbveränderung in der Gruppe PF15\% $(p=<0,0001)$ und auch in der Gruppe XtraBoost $(p=0,0004)$. Somit kommt es in beiden Bleichgruppen zu einem deutlichen Rückgang der Bleichwirkung (Abb 5.10, Tab 5.10).

Tabelle 5.10: Veränderung $L^{*}$-Wert $\mathrm{t} 3$ zu t4

\begin{tabular}{|l|l|l|l|l|}
\hline $\mathrm{L}^{*}$ & $\begin{array}{l}\text { Mittelwert } \\
\text { T3 } \mathrm{L} \pm \mathrm{s}\end{array}$ & $\begin{array}{l}\text { Mittelwert } \\
\text { T4 } \mathrm{L} \pm \mathrm{s}\end{array}$ & $\begin{array}{l}\mathrm{L} \pm \mathrm{s} \\
\mathrm{P}\end{array}$ \\
\hline Kontrollgruppe & $70,15 \pm 3,27$ & $69,44 \pm 3,07$ & $0,72 \pm 2,57$ & 0,7056 \\
\hline PF 15\% & $73,05 \pm 3,27$ & $69,55 \pm 2,97$ & $3,49 \pm 2,35$ & $<0,0001$ \\
\hline Xtra Boost 38\% & $72,10 \pm 3,33$ & $69,91 \pm 2,8$ & $2,19 \pm 2,20$ & 0,0004 \\
\hline
\end{tabular}

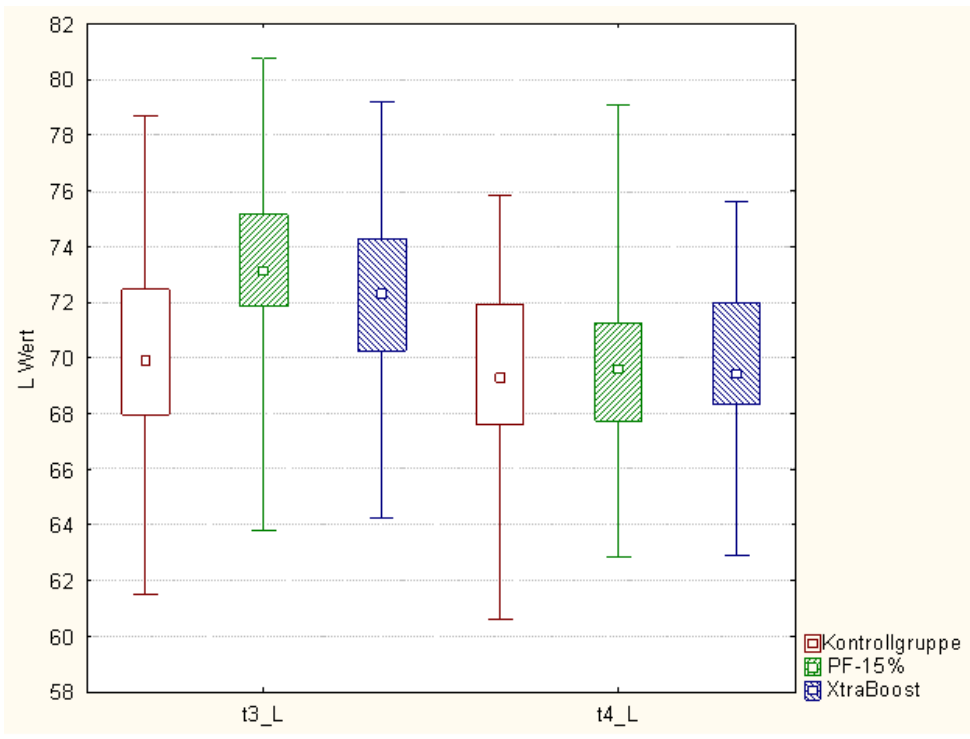

Abbildung 5.10: Veränderung des $L^{*}$-Wertes t3 zu t4 
$a^{*}$-Wert

Im letzten Zeitintervall, t3 zu t4, stellt die Messung eine signifikante Veränderung des $\mathrm{a}^{*}$ Wertes in der Kontrollgruppe ( $p=0,0006)$ fest. Bei den Gruppen PF15\% und XtraBoost wurde dagegen keine Farbänderung festgestellt, das heißt, bis zum Versuchsende bleibt der $a^{*}$ Wert signifikant niedriger, und die Aufhellung der Rötlichkeit bleibt stabil ( $p=1,0000$ und $p=0,3635)($ Abb 5.11, Tab 5.11).

Tabelle 5.11: Veränderung $a^{*}$-Wert t3 zu t4

\begin{tabular}{|l|l|l|l|l|}
\hline$a^{*}$ & $\begin{array}{l}\text { Mittelwert } \\
\text { T3a } \pm \mathrm{s}\end{array}$ & $\begin{array}{l}\text { Mittelwert } \\
\text { T4a } \pm \mathrm{s}\end{array}$ & $\Delta \mathrm{a} \pm \mathrm{s}$ & $\mathrm{P}$ \\
\hline Kontrollgruppe & $2,96 \pm 1,68$ & $1,95 \pm 1,20$ & $1,01 \pm 0,87$ & 0,0006 \\
\hline PF15\% & $1,28 \pm 1,42$ & $1,26 \pm 1,28$ & $0,03 \pm 0,46$ & 1,000 \\
\hline XtraBoost 38\% & $1,13 \pm 1,52$ & $0,68 \pm 1,29$ & $0,45 \pm 0,64$ & 0,3635 \\
\hline
\end{tabular}

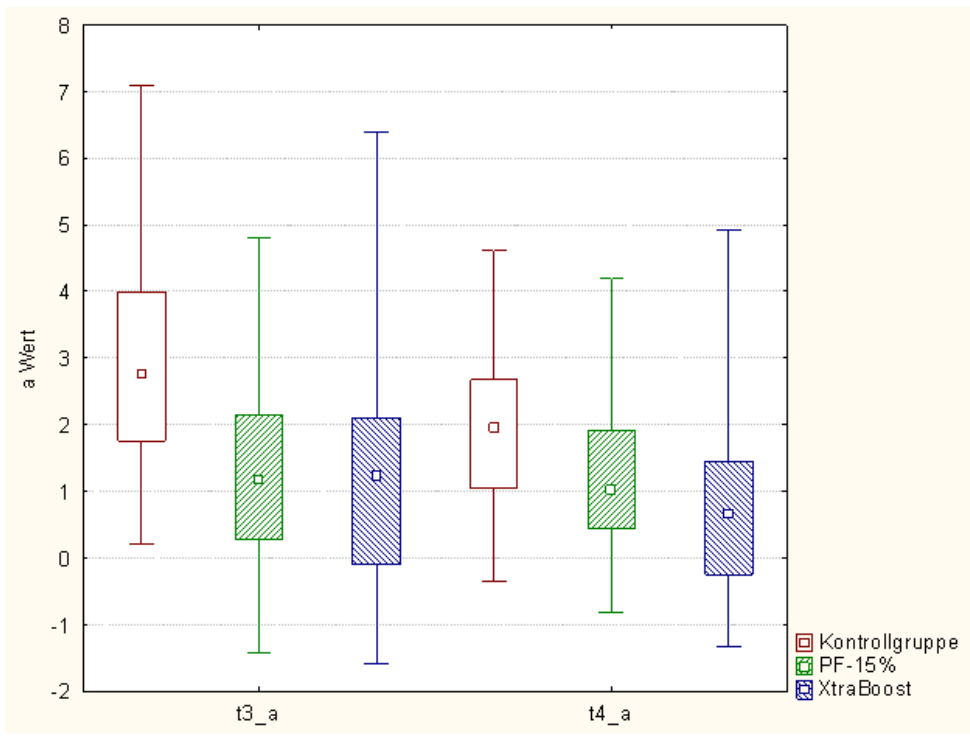

Abbildung 5.11: Veränderung des $a^{*}$-Wertes t3 zu t4 
$b^{*}$-Wert

Im letzten Zeitintervall, t3 zu t4, zeigt die Statistik eine signifikante Veränderung der b*-Werte innerhalb der Kontrollgruppe und der Gruppe XtraBoost an $(p<0,0001)$, das heißt, die Gelblichkeit der Zähne nahm ab. In der PF15\%-Gruppe war dies nicht der Fall $(p=0,1356)$ (Abb 5.12, Tab 5.12).

Tabelle 5.12: Veränderung $b^{*}$-Wert $\mathrm{t} 3$ zu t4

\begin{tabular}{|l|l|l|l|l|}
\hline $\mathrm{b}^{*}$ & $\begin{array}{l}\text { Mittelwert } \\
\text { T3 } \mathrm{b} \pm \mathrm{s}\end{array}$ & $\begin{array}{l}\text { Mittelwert } \\
\text { T4 } \mathrm{b} \pm \mathrm{s}\end{array}$ & $\mathrm{P} \pm \mathrm{s}$ & $<0,0001$ \\
\hline Kontrollgruppe & $15,85 \pm 2,80$ & $12,80 \pm 2,52$ & $3,05 \pm 2,16$ & 0,1356 \\
\hline PF 15\% & $13,37 \pm 2,48$ & $12,41 \pm 2,15$ & $0,96 \pm 1,20$ & $<0,0001$ \\
\hline XtraBoost 38\% & $14,87 \pm 2,58$ & $12,65 \pm 2,07$ & $2,22 \pm 1,52$ & \\
\hline
\end{tabular}

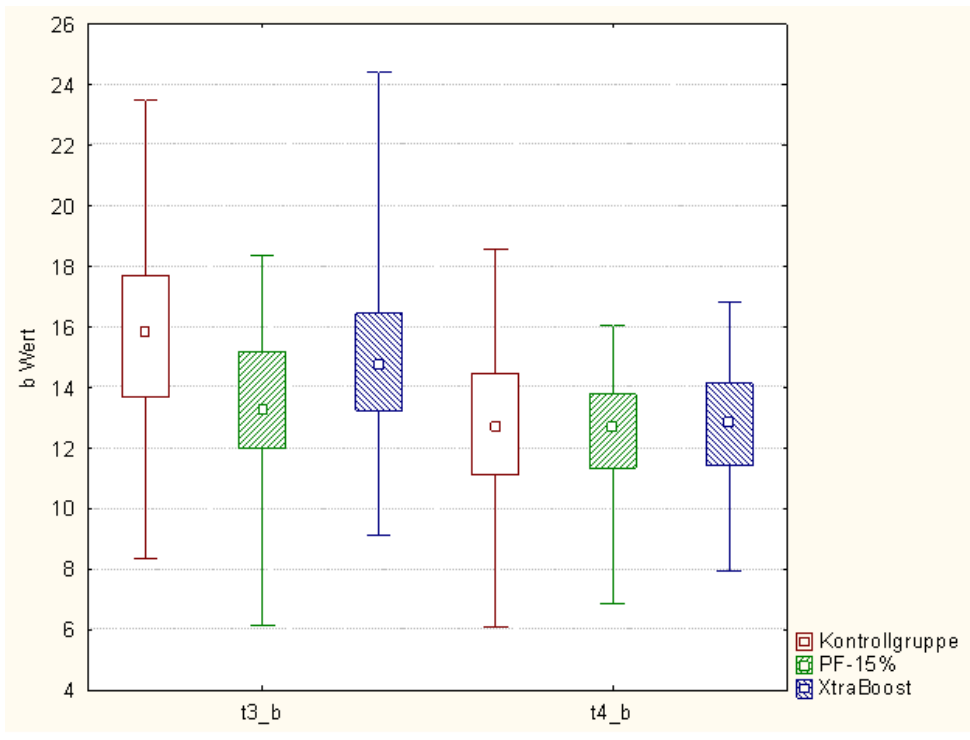

Abbildung 5.12: Veränderung des $b^{*}$-Wertes $\mathrm{t} 3$ zu t4 


\subsection{Verlauf: Baseline zu t4}

$L^{*}$-Wert

Da der L*-Wert mit der Helligkeit eines Objektes assoziiert ist (Kap 2.3), lässt ein Rückgang des $L^{*}$-Wertes auf ein Verblassen der Helligkeit und somit der Bleichwirkung schließen. Während bei der Gruppe PF15\% zum Zeitpunkt t1, t2 und t3 die $L^{*}$-Werte im Vergleich zum Baseline-Wert noch signifikant erhöht waren, gibt es nach 6 Monaten keinen statistischen Unterschied mehr $(p=0,1288)$.

In der Gruppe XtraBoost ist der $L^{*}$-Vergleich zwischen Baseline zu t1, t2, t3 und t4 Werten stets signifikant, das heißt, es kommt nicht zu einem vollständigen Rückgang der Aufhellung bis hin zum Ausgangswert. Die $L^{*}$-Werte sind im Verlauf der Zeitpunkte t1 zu t3 nicht signifikant gesunken $(p=<0,1939)$. Man erkennt jedoch auch in der XtraBoost-Gruppe trotz des noch signifikanten Unterschieds der Baseline-Werte zu den t4 Werten eine starke Tendenz der Helligkeit hin zum Baseline-Wert. Das heißt, auch in der XtraBoost-Gruppe wird ein Rückgang der Aufhellung verzeichnet (Abb 5.13, Tab 5.13, Tab 5.14, Tab 5.15).

Tabelle 5.13: Veränderung L*-Wert Baseline zu t4

\begin{tabular}{|l|l|l|l|l|}
\hline $\mathrm{L}^{*}$ & $\begin{array}{l}\text { Mittelwert } \\
\text { Baseline } \\
\mathrm{L} \pm \mathrm{s}\end{array}$ & $\begin{array}{l}\text { Mittelwert } \\
\mathrm{T} 4 \mathrm{~L} \pm \mathrm{s}\end{array}$ & $\Delta \mathrm{L} \pm \mathrm{S}$ & $\begin{array}{l}\text { Signifikanzniveau } \\
\mathrm{P}\end{array}$ \\
\hline Kontrollgruppe & $68,16 \pm 3,84$ & $69,44 \pm 3,07$ & $1,28 \pm 3,6$ & 0,1558 \\
\hline PF 15\% & $68,23 \pm 3,79$ & $69,55 \pm 2,97$ & $1,32 \pm 3,21$ & 0,1288 \\
\hline Xtra Boost 38\% & $68,32 \pm 3,7$ & $69,91 \pm 2,8$ & $1,59 \pm 3,08$ & 0,0230 \\
\hline
\end{tabular}

Tabelle 5.14: Veränderung $L^{*}$-Wert $\mathrm{t} 1$ zu t4

\begin{tabular}{|l|l|l|l|l|}
\hline $\mathrm{L}^{*}$ & $\begin{array}{l}\text { Mittelwert } \\
\mathrm{T} 1 \\
\mathrm{~L} \pm \mathrm{s}\end{array}$ & $\begin{array}{l}\text { Mittelwert } \\
\mathrm{T} 4 \\
\mathrm{~L} \pm \mathrm{s}\end{array}$ & $\Delta \mathrm{L} \pm \mathrm{s}$ & $\begin{array}{l}\text { Signifikanzniveau } \\
\mathrm{P}\end{array}$ \\
\hline Kontrollgruppe & $69,69 \pm 3,6$ & $69,44 \pm 3,07$ & $0,25 \pm 3,45$ & 0,9919 \\
\hline PF 15\% & $72,48 \pm 3,87$ & $69,55 \pm 2,97$ & $2,93 \pm 4,91$ & $<0,0001$ \\
\hline Xtra Boost 38\% & $73,25 \pm 3,35$ & $69,91 \pm 2,8$ & $3,23 \pm 3,96$ & $<0,0001$ \\
\hline
\end{tabular}


Tabelle 5.15 p Werte adjustiert nach Tukey zum Vergleich der $L^{*}$-Werte innerhalb der Zeitintervalle Baseline $-\mathrm{t} 4$.

Statistisch signifikante Unterschiede liegen bei $p<0,05$ und sind mit * gekennzeichnet.

\begin{tabular}{|c|c|c|c|c|c|c|}
\hline Kontrollgruppe & Zeitpunkte & Baseline & $T 1$ & $T 2$ & T3 & T4 \\
\hline & Baseline & & 0.0531 & $0.0285^{*}$ & $0.0039^{*}$ & 0.1558 \\
\hline & $\mathrm{T} 1$ & 0.0531 & & 0.9995 & 0.9205 & 0.9919 \\
\hline & T2 & $0.0285^{*}$ & 0.9995 & & 0.9735 & 0.9633 \\
\hline & T3 & $0.0039^{*}$ & 0.9205 & 0.9735 & & 0.7056 \\
\hline & T4 & 0.1558 & 0.9919 & 0.9633 & 0.7056 & \\
\hline \multirow[t]{6}{*}{ PF $15 \%$} & Zeitpunkte & Baseline & $T 1$ & $T 2$ & T3 & T4 \\
\hline & Baseline & & $<.0001^{*}$ & $<.0001^{*}$ & $<.0001^{*}$ & 0.1288 \\
\hline & T1 & $<.0001^{*}$ & & $0.0246^{*}$ & 0.8537 & $<.0001^{*}$ \\
\hline & T2 & $<.0001^{*}$ & $0.0246^{*}$ & & $0.2712^{*}$ & $<.0001^{*}$ \\
\hline & T3 & $<.0001^{*}$ & 0.8537 & 0.2712 & & $<.0001^{*}$ \\
\hline & T4 & 0.1288 & $<.0001^{*}$ & $<.0001^{*}$ & $<.0001^{*}$ & \\
\hline \multirow[t]{6}{*}{ XtraBoost } & Zeitpunkte & Baseline & $T 1$ & $T 2$ & T3 & T4 \\
\hline & Baseline & & $<.0001^{*}$ & $<.0001^{*}$ & $<.0001^{*}$ & $0.0230^{*}$ \\
\hline & $\mathrm{T} 1$ & $<.0001^{*}$ & & 0.0741 & 0.1939 & $<.0001^{*}$ \\
\hline & T2 & $<.0001^{*}$ & 0.0741 & & 0.9933 & $0.0022^{*}$ \\
\hline & T3 & $<.0001^{*}$ & 0.1939 & 0.9933 & & $0.0004^{*}$ \\
\hline & $\mathrm{T} 4$ & $0.0230^{*}$ & $<.0001^{*}$ & $0.0022^{*}$ & $0.0004^{*}$ & \\
\hline
\end{tabular}

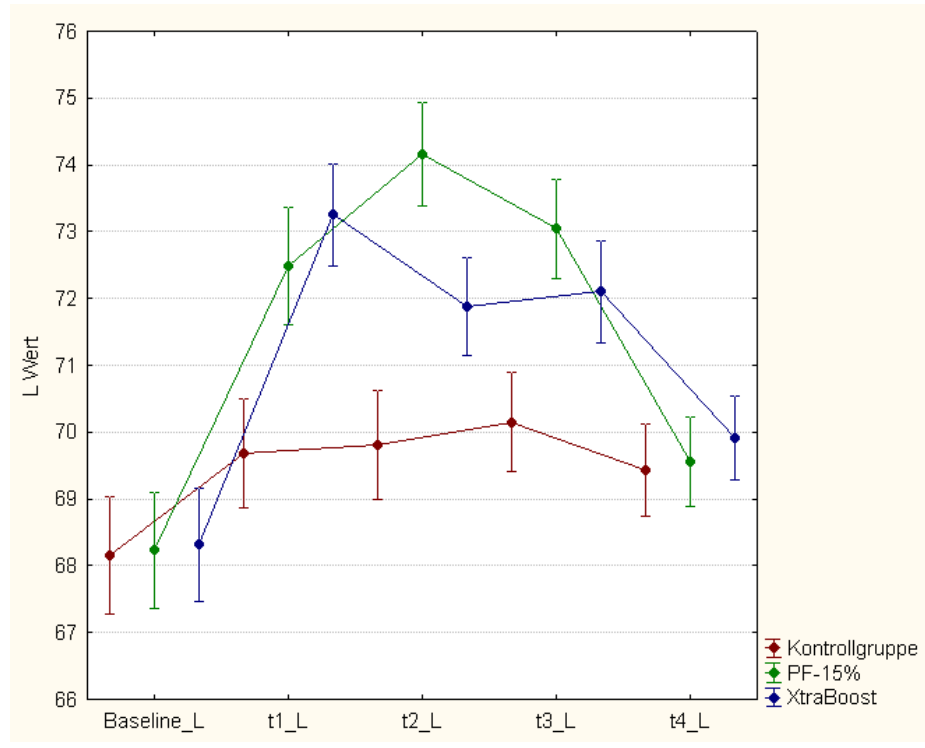

Abbildung 5.13:Verlauf des $L^{*}$-Wertes über die Zeit 
$a^{*}$-Wert

Bei Betrachtung der $\mathrm{a}^{*}$-Werte erkennt man in beiden Versuchsgruppen PF15\% und XtraBoost eine signifikante Farbveränderung der Baseline-Werte im Vergleich mit dem Zeitpunkt t4 $(p<0,0001)$. Im Vergleich t1 zu t4 lässt sich keine Veränderung des $a^{*}$-Wertes in der Gruppe PF15\% nachweisen. In der Gruppe XtraBoost reduziert sich der a*-Wert kontinuierlich, was einer Abnahme der Rötlichkeit entspricht. Im Vergleich der direkt aufeinander folgenden Zeitpunkte kann man zwar keinen statistischen Unterschied feststellen, aber wenn man die Zeitintervalle vergrößert und t1 mit $t 3(p=0,0287)$ oder t2 mit $t 4(p=0,0071)$ vergleicht, sind die Unterschiede der $a^{*}$-Werte signifikant, das heißt, die Zähne sind in der Rötlichkeit aufgehellt.

Im letzten Zeitintervall t3 zu t4 kommt es auch zu einer signifikanten Veränderung des $a^{*}$ Wertes in der Kontrollgruppe $(p=0,0006)$. Betrachtet man jedoch das Zeitintervall des Gesamtversuches (Baseline zu t4), ist keine signifikante Veränderung der Rötlichkeit in der Kontrollgruppe festzustellen ( $p=0,2746)$ (Abb 5.14, Tab 5.16, Tab 5.17, Tab 5.18).

Tabelle 5.16: Veränderung a*-Wert Baseline zu t4

\begin{tabular}{|l|l|l|l|l|}
\hline$a^{*}$ & $\begin{array}{l}\text { Mittelwert } \\
\text { Baseline } \\
\mathrm{a} \pm \mathrm{s}\end{array}$ & $\begin{array}{l}\text { Mittelwert } \\
\text { T4 a } \pm \mathrm{s}\end{array}$ & $\Delta \mathrm{a} \pm \mathrm{s}$ & $\mathrm{P}$ \\
\hline Kontrollgruppe & $2,45 \pm 1,50$ & $1,95 \pm 1,20$ & $0,5 \pm 0,78$ & 0,2746 \\
\hline PF15\% & $2,65 \pm 1,85$ & $1,26 \pm 1,28$ & $1,39 \pm 1,10$ & $<0,0001$ \\
\hline XtraBoost 38\% & $2,55 \pm 1,65$ & $0,68 \quad \pm \quad 1,29$ & $1,87 \pm 0,98$ & $<0,0001$ \\
\hline
\end{tabular}

Tabelle 5.17: Veränderung $a^{*}$-Wert t1 zu t4

\begin{tabular}{|l|l|l|l|l|}
\hline $\mathrm{a}^{*}$ & $\begin{array}{l}\text { Mittelwert } \\
\mathrm{T} 1 \\
\mathrm{a} \pm \mathrm{s}\end{array}$ & $\begin{array}{l}\text { Mittelwert } \\
\mathrm{T} 4 \\
\mathrm{~A} \pm \mathrm{s}\end{array}$ & $\Delta \mathrm{a} \pm \mathrm{s}$ & $\mathrm{P}$ \\
\hline Kontrollgruppe & $2,29 \pm 1,61$ & $1,95 \pm 1,20$ & $0,34 \pm 0,80$ & 0,0011 \\
\hline PF15\% & $1,84 \pm 1,61$ & $1,26 \pm 1,28$ & $0,58 \pm 0,75$ & 0,1345 \\
\hline XtraBoost 38\% & $1,87 \pm 1,87$ & $0,68 \quad \pm \quad 1,29$ & $1,19 \pm 1,01$ & $<0,0001$ \\
\hline
\end{tabular}


Tabelle 5.18: $p$ Werte adjustiert nach Tukey zum Vergleich der $a^{*}$-Werte innerhalb der Zeitintervalle Baseline - t4.

Statistisch signifikante Unterschiede liegen bei $p<0,05$ und sind mit * gekennzeichnet.

\begin{tabular}{|c|c|c|c|c|c|c|}
\hline Kontroll- & Zeitpunk- & Baseline & $T 1$ & $T 2$ & T3 & T4 \\
\hline & Baseline & & 0.3155 & 0.0947 & 0.2439 & 0.2746 \\
\hline & $\mathrm{T} 1$ & 0.3155 & & 0.9773 & 0.9999 & $0.0011^{*}$ \\
\hline & $\mathrm{T} 2$ & 0.0947 & 0.9773 & & 0.9922 & $<.0001^{*}$ \\
\hline & T3 & 0.2439 & 0.9999 & 0.9922 & & $0.0006^{*}$ \\
\hline & $\mathrm{T} 4$ & 0.2746 & $0.0011^{*}$ & $<.0001^{*}$ & $0.0006^{*}$ & \\
\hline \multirow[t]{6}{*}{ PF $15 \%$} & Zeitpunk- & Baseline & $T 1$ & $T 2$ & T3 & T4 \\
\hline & Baseline & & $0.0108^{*}$ & $<.0001^{*}$ & $<.0001^{*}$ & $<.0001^{*}$ \\
\hline & $\mathrm{T} 1$ & $0.0108^{*}$ & & 0.5928 & 0.1699 & 0.1345 \\
\hline & $\mathrm{T} 2$ & $<.0001^{*}$ & 0.5928 & & 0.9367 & 0.9020 \\
\hline & T3 & $<.0001^{*}$ & 0.1699 & 0.9367 & & 1.0000 \\
\hline & $\mathrm{T} 4$ & $<.0001^{*}$ & 0.1345 & 0.9020 & 1.0000 & \\
\hline \multirow[t]{6}{*}{ XtraBoost } & Zeitpunk- & Baseline & $T 1$ & $T 2$ & T3 & T4 \\
\hline & Baseline & & $0.0481^{*}$ & $0.0004^{*}$ & $<.0001^{*}$ & $<.0001^{*}$ \\
\hline & $\mathrm{T} 1$ & $0.0481^{*}$ & & 0.6428 & $0.0287^{*}$ & $<.0001^{*}$ \\
\hline & $\mathrm{T} 2$ & $0.0004^{*}$ & 0.6428 & & 0.5235 & $0.0071^{*}$ \\
\hline & T3 & $<.0001^{*}$ & $0.0287^{*}$ & 0.5235 & & 0.3635 \\
\hline & $\mathrm{T} 4$ & $<.0001^{*}$ & $<.0001^{*}$ & $0.0071^{*}$ & 0.3635 & \\
\hline
\end{tabular}

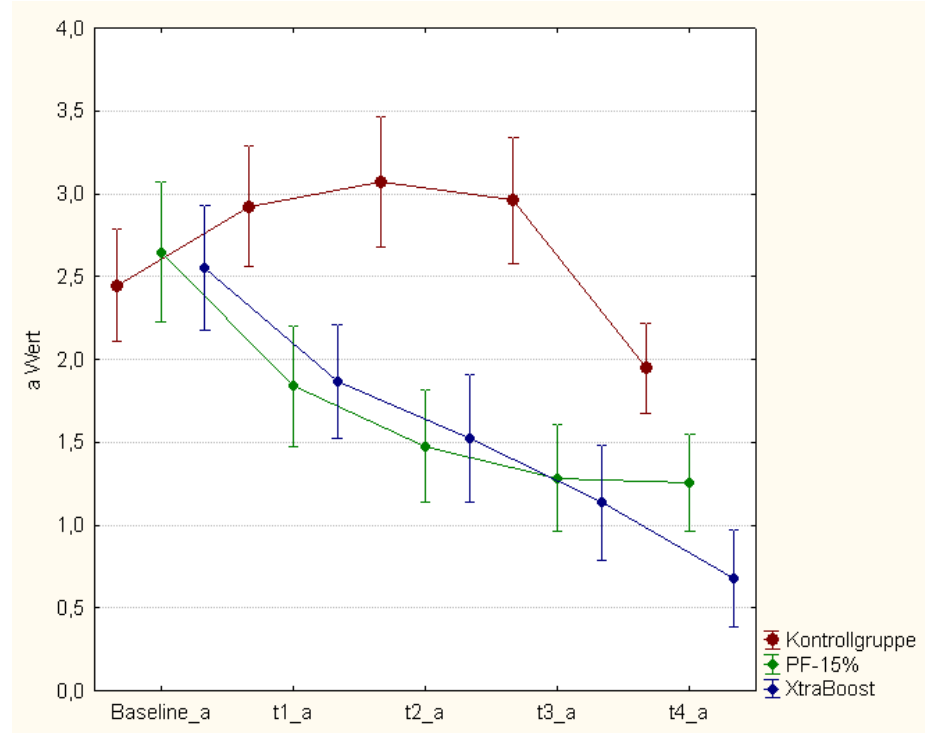

Abbildung 5.14:Verlauf des $a^{*}$-Wertes über die Zeit 
$b^{*}$-Wert

Der b*-Wert ist mit der Gelblichkeit eines Objektes assoziiert (Kap 2.3), das heißt, ein Rückgang des $b^{*}$-Wertes lässt auf eine Abnahme der Gelblichkeit des Zahnes schließen. In der Kontrollgruppe kam es im letzten Zeitintervall ( $\mathrm{t} 3 \mathrm{zu}$ t4) zu einem starken Abfall des b*Wertes $(p<0,0001)$ und somit zu einer Aufhellung der Gelblichkeit der Zähne. Daher waren auch alle Zeitvergleiche zu den t4 Werten entsprechend signifikant verändert.

Bei allen anderen Vergleichen innerhalb der Kontrollgruppe gab es keine statistisch feststellbare Veränderung des $b^{*}$-Wertes. In der PF15\%-Gruppe fiel der $b^{*}$-Wert bis zur Messung t2 signifikant $(p<0,0001)$ ab. Da zwischen den Messungen $t 2$ und $t 3(p=0,9479)$ sowie $t 3$ und $t 4$ $(p=0,1356)$ keine signifikante Veränderung des $b^{*}$-Wertes verzeichnet wurden, kann man davon ausgehen, dass der Wert bis zum Ende der Studie auf diesem Niveau verblieb.

In der Gruppe XtraBoost reduziert sich der $b^{*}$-Wert, genau wie der $a^{*}$-Wert, kontinuierlich, was einer Aufhellung der Gelblichkeit entspricht. Im Vergleich der direkt aufeinander folgenden Zeitpunkte kann man keinen statistischen Unterschied feststellen. Wenn jedoch die Zeitintervalle vergrößert werden und Baseline mit $t 3(p=0,0007)$ oder mit $t 4(p<0,0001)$ verglichen wird, sind die Unterschiede der $b^{*}$-Werte signifikant (Abb 5.15, Tab 5.19, Tab 5.20, Tab 5.21), das heißt, die Gelblichkeit der Zähne nimmt ab.

Tabelle 5.19: Veränderung $b^{*}$-Wert Baseline zu t4

\begin{tabular}{|l|l|l|l|l|}
\hline $\mathrm{b}^{*}$ & $\begin{array}{l}\text { Mittelwert } \\
\text { Baseline } \\
\mathrm{b} \pm \mathrm{s}\end{array}$ & $\begin{array}{l}\text { Mittelwert } \\
\mathrm{T} 4 \mathrm{~b} \pm \mathrm{s}\end{array}$ & $\Delta \mathrm{b} \pm \mathrm{s}$ & $\mathrm{P}$ \\
\hline Kontrollgruppe & $16,14 \pm 3,13$ & $12,80 \pm 2,52$ & $3,33 \pm 2,19$ & $<0,0001$ \\
\hline PF 15\% & $16,59 \pm 2,99$ & $12,41 \pm 2,15$ & $4,18 \pm 2,07$ & $<0,0001$ \\
\hline XtraBoost 38\% & $16,47 \pm 3,07$ & $12,65 \pm 2,07$ & $3,83 \pm 1,7$ & $<0,0001$ \\
\hline
\end{tabular}

Tabelle 5.20: Veränderung b*-Wert Baseline zu t4

\begin{tabular}{|l|l|l|l|l|}
\hline $\mathrm{b}^{*}$ & $\begin{array}{l}\text { Mittelwert } \\
\text { T1 } \\
\mathrm{b} \pm \mathrm{s}\end{array}$ & $\begin{array}{l}\text { Mittelwert } \\
\mathrm{b} \pm \mathrm{s}\end{array}$ & $\Delta \mathrm{b} \pm \mathrm{s}$ & $\mathrm{P}$ \\
\hline Kontrollgruppe & $15,31 \pm 2,59$ & $12,80 \pm 2,52$ & $2,51 \pm 1,83$ & $<0,0001$ \\
\hline PF 15\% & $15,03 \pm 2,55$ & $12,41 \pm 2,15$ & $2,62 \pm 1,58$ & $<0,0001$ \\
\hline XtraBoost 38\% & $15,42 \pm 2,21$ & $12,65 \pm 2,07$ & $2,77 \pm 1,55$ & $<0,0001$ \\
\hline
\end{tabular}


Tabelle 5.21: $p$ Werte adjustiert nach Tukey zum Vergleich der $b^{*}$-Werte innerhalb der Zeitintervalle Baseline $-\mathrm{t} 4$.

Statistisch signifikante Unterschiede liegen bei $p<0,05$ und sind mit * gekennzeichnet.

\begin{tabular}{|c|c|c|c|c|c|c|}
\hline Kontroll- & Zeitpunk- & Baseline & $T 1$ & $T 2$ & T3 & T4 \\
\hline & Baseline & & 0.3603 & 1.0000 & 0.9712 & $<.0001^{*}$ \\
\hline & $\mathrm{T} 1$ & 0.3603 & & 0.3636 & 0.7503 & $<.0001^{*}$ \\
\hline & $\mathrm{T} 2$ & 1.0000 & 0.3636 & & 0.9722 & $<.0001^{*}$ \\
\hline & T3 & 0.9712 & 0.7503 & 0.9722 & & $<.0001^{*}$ \\
\hline & $\mathrm{T} 4$ & $<.0001^{*}$ & $<.0001^{*}$ & $<.0001^{*}$ & $<.0001^{*}$ & \\
\hline \multirow[t]{6}{*}{ PF $15 \%$} & Zeitpunk- & Baseline & $T 1$ & $T 2$ & T3 & T4 \\
\hline & Baseline & & $0.0015^{*}$ & $<.0001^{*}$ & $<.0001^{*}$ & $<.0001^{*}$ \\
\hline & T1 & $0.0015^{\star}$ & & $<.0001^{*}$ & $0.0007^{*}$ & $<.0001^{*}$ \\
\hline & $\mathrm{T} 2$ & $<.0001^{*}$ & $<.0001^{*}$ & & 0.9479 & 0.4978 \\
\hline & T3 & $<.0001^{*}$ & $0.0007^{*}$ & 0.9479 & & 0.1356 \\
\hline & $\mathrm{T} 4$ & $<.0001^{*}$ & $<.0001^{*}$ & 0.4978 & 0.1356 & \\
\hline \multirow[t]{6}{*}{ XtraBoost } & Zeitpunk- & Baseline & $T 1$ & $T 2$ & T3 & T4 \\
\hline & Baseline & & 0.0623 & $0.0178^{*}$ & $0.0007^{*}$ & $<.0001^{*}$ \\
\hline & $\mathrm{T} 1$ & 0.0623 & & 0.9922 & 0.6483 & $<.0001^{*}$ \\
\hline & $\mathrm{T} 2$ & $0.0178^{*}$ & 0.9922 & & 0.8856 & $<.0001^{*}$ \\
\hline & T3 & $0.0007^{*}$ & 0.6483 & 0.8856 & & $<.0001^{*}$ \\
\hline & $\mathrm{T} 4$ & $<.0001^{*}$ & $<.0001^{*}$ & $<.0001^{*}$ & $<.0001^{*}$ & \\
\hline
\end{tabular}

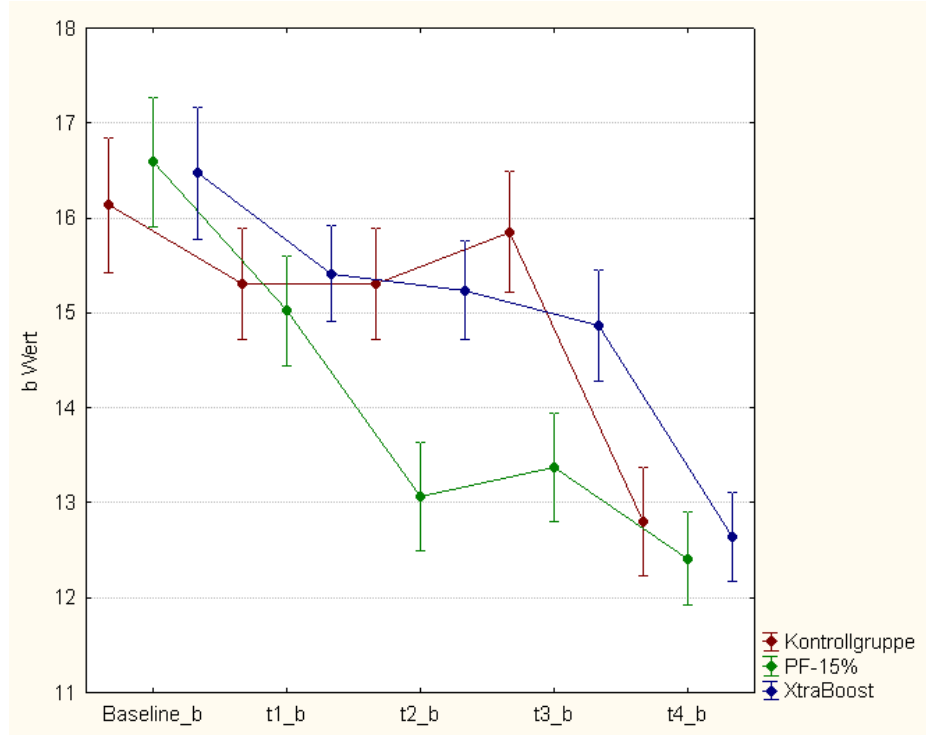

Abbildung 5.15:Verlauf des $b^{*}$-Wertes über die Zeit 


\subsection{Gruppenvergleich über den zeitlichen Verlauf}

$L^{*}$-Wert

Da die ausgewählten Zähne über ein an dem L*-Wert orientiertes Randomisierungsverfahren den drei Gruppen zugeordnet wurden, war zu erwarten, dass zu Beginn der Untersuchung (Baseline) der $L^{*}$-Wert in allen drei Gruppen annähernd gleich war. Dies wurde auch durch die statistische Auswertung bestätigt, die keinen signifikanten Unterschied zwischen den Gruppen erkennen ließ ( $p=0,9313$ - 0,9999).

Der Vergleich der drei Gruppen untereinander unmittelbar nach der Bleiche (t1) zeigt eine signifikante $L^{*}$-Wert Veränderung der Gruppen PF15\% ( $\left.p<0,001\right)$ und XtraBoost $(p<0,001)$ zur Kontrollgruppe. Dies belegt die Wirksamkeit der beiden Bleichungen, beide Bleichgruppen PF15\% und XtraBoost waren gegenüber der Kontrollgruppe deutlich aufgehellt. Im Vergleich der beiden Bleichverfahren untereinander war wiederum kein statistischer Unterschied $(p=0,3860)$ feststellbar, was auf eine ähnliche Bleichwirkung schließen lässt. Zum letzten Zeitpunkt t4 konnte kein signifikanter Unterschied der $L^{*}$-Werte zwischen den drei Gruppen mehr festgestellt werden, das heißt, die Bleichungsaufhellung der Zähne war stark zurückgegangen. Die Signifikanzniveaus der Gruppe PF15\% und XtraBoost zur ungebleichten Kontrollgruppe betrugen hier $p=0,9664$ bzw. $p=0,4647$ (Tab 5.22). 
Tabelle 5.22 p Werte adjustiert nach Tukey zum Vergleich der $L^{*}$-Werte innerhalb der Gruppen zu den unterschiedlichen Zeitpunkten.

Statistisch signifikante Unterschiede liegen bei $p<0,05$ und sind mit * gekennzeichnet.

\begin{tabular}{|c|c|c|c|c|}
\hline Gruppe & Baseline_L & Kontrollgruppe & PF $15 \%$ & XtraBoost $38 \%$ \\
\hline Kontrollgruppe & 68.1593583 & & 0.9999 & 0.9344 \\
\hline PF $15 \%$ & 68.1535088 & 0.9999 & & 0.9313 \\
\hline XtraBoost $38 \%$ & 68.3729790 & 0.9344 & 0.9313 & \\
\hline Gruppe & $T 1 \_L$ & Kontrollgruppe & PF $15 \%$ & XtraBoost $38 \%$ \\
\hline Kontrollgruppe & 69.6851710 & & $<.0001^{*}$ & $<.0001^{*}$ \\
\hline PF $15 \%$ & 72.4811765 & $<.0001^{*}$ & & 0.3860 \\
\hline XtraBoost $38 \%$ & 73.2542828 & $<.0001^{*}$ & 0.3860 & \\
\hline Gruppe & $T 2 \_L$ & Kontrollgruppe & PF $15 \%$ & XtraBoost $38 \%$ \\
\hline Kontrollgruppe & 69.8100331 & & $<.0001^{*}$ & $0.0003^{*}$ \\
\hline PF $15 \%$ & 74.2084967 & $<.0001^{*}$ & & $0.0002^{*}$ \\
\hline XtraBoost $38 \%$ & 71.9689026 & $0.0003^{*}$ & $0.0002^{*}$ & \\
\hline Gruppe & $t 3 \_L$ & Kontrollgruppe & PF $15 \%$ & XtraBoost $38 \%$ \\
\hline Kontrollgruppe & 70.1522621 & & $<.0001^{*}$ & $0.0004^{*}$ \\
\hline PF $15 \%$ & 73.0573787 & $<.0001^{*}$ & & 0.2430 \\
\hline XtraBoost $38 \%$ & 72.2050224 & $0.0004^{*}$ & 0.2430 & \\
\hline Gruppe & $t 4 \_L$ & Kontrollgruppe & PF $15 \%$ & XtraBoost $38 \%$ \\
\hline Kontrollgruppe & 69.4354809 & & 0.9664 & 0.4647 \\
\hline PF $15 \%$ & 69.5535088 & 0.9664 & & 0.6215 \\
\hline XtraBoost $38 \%$ & 69.9960784 & 0.4647 & 0.6215 & \\
\hline
\end{tabular}


$a^{*}$-Wert

Zu Beginn der Untersuchung vor dem Bleichen (Baseline), war der $\mathrm{a}^{*}$-Wert in allen drei Gruppen annähernd gleich, sodass sich kein signifikanter Unterschied zwischen den Gruppen widerspiegelt $(p=0,7327, p=0,9176$ und $p=0,9322)$.

Der Vergleich der drei Gruppen untereinander unmittelbar nach der Bleiche (t1) zeigt eine signifikante $a^{*}$-Wert-Veränderung der Gruppen PF15\% $(p<0,001)$ und XtraBoost $(p<0,001)$ zur Kontrollgruppe. Dies belegt, dass es auch in Bezug auf die Rötlichkeit gelungen ist, durch die Bleichung eine deutliche Farbaufhellung in beiden Bleichgruppen zu erreichen. Im Vergleich der beiden Bleichverfahren untereinander ist kein statistischer Unterschied $(p=0,9934)$ erkennbar, was wiederum auf eine nahezu gleiche Bleichwirkung schließen lässt. Anders als beim $L^{*}$-Wert bleibt eine signifikante Farbveränderung des $a^{*}$-Wertes über den gesamten Versuchszeitraum bestehen, insbesondere kommt es auch nach 6 Monaten (t4) nicht zu einer vollständigen Annährung der $a^{*}$-Werte beider Bleichgruppen, PF15\% und XtraBoost, an die Kontrollgruppe (Tab 5.23). 
Tabelle 5.23 p Werte adjustiert nach Tukey zum Vergleich der $a^{*}$-Werte innerhalb der Gruppen zu den unterschiedlichen Zeitpunkten.

Statistisch signifikante Unterschiede liegen bei $p<0,05$ und sind mit * gekennzeichnet.

\begin{tabular}{|c|c|c|c|c|}
\hline Gruppe & Baseline_a & Kontrollgruppe & PF $15 \%$ & XtraBoost $38 \%$ \\
\hline Kontrollgruppe & 2.44514388 & & 0.7327 & 0.9176 \\
\hline PF $15 \%$ & 2.64763942 & 0.7327 & & 0.9322 \\
\hline XtraBoost $38 \%$ & 2.55152534 & 0.9176 & 0.9322 & \\
\hline Gruppe & $t 1 \_a$ & Kontrollgruppe & PF $15 \%$ & XtraBoost $38 \%$ \\
\hline Kontrollgruppe & 2.92331042 & & $<.0001^{*}$ & $0.0001^{*}$ \\
\hline PF $15 \%$ & 1.83814617 & $<.0001^{*}$ & & 0.9934 \\
\hline XtraBoost $38 \%$ & 1.86593328 & $0.0001^{*}$ & 0.9934 & \\
\hline Gruppe & t2_a & Kontrollgruppe & PF $15 \%$ & XtraBoost $38 \%$ \\
\hline Kontrollgruppe & 3.06998727 & & $<.0001^{*}$ & $<.0001^{*}$ \\
\hline PF $15 \%$ & 1.47654698 & $<.0001^{*}$ & & 0.9832 \\
\hline XtraBoost $38 \%$ & 1.52323911 & $<.0001^{*}$ & 0.9832 & \\
\hline Gruppe & t3_a & Kontrollgruppe & PF $15 \%$ & XtraBoost $38 \%$ \\
\hline Kontrollgruppe & 2.95953145 & & $<.0001$ & $<.0001$ \\
\hline PF $15 \%$ & 1.28301502 & $<.0001^{*}$ & & 0.8226 \\
\hline XtraBoost $38 \%$ & 1.13478992 & $<.0001^{*}$ & 0.8226 & \\
\hline Gruppe & t4_a & Kontrollgruppe & PF $15 \%$ & XtraBoost $38 \%$ \\
\hline Kontrollgruppe & 1.94697224 & & $0.0022^{*}$ & $<.0001^{*}$ \\
\hline PF $15 \%$ & 1.25624650 & $0.0022^{*}$ & & $0.0138^{*}$ \\
\hline XtraBoost $38 \%$ & 0.68184365 & $<.0001^{*}$ & $0.0138^{*}$ & \\
\hline
\end{tabular}


$b^{*}$-Wert

Zu Beginn der Untersuchung vor dem Bleichen (Baseline) war auch der $b^{*}$-Wert in allen drei Gruppen annähernd gleich, sodass sich kein signifikanter Unterschied zwischen den Gruppen widerspiegelt ( $p=0,6275, p=0,7739$ und $p=0,9691)$. Zum Zeitpunkt t1 zeigt sich im Wesentlichen das gleiche Bild, keine signifikanten Veränderungen zwischen den drei Versuchsgruppen. Zum Zeitpunkt t2 hat sich der b*-Wert nur in der PF15\%-Gruppe signifikant zu den beiden anderen verändert (beide $p<0,0001$ ), zwischen der XtraBoost-Gruppe und der Kontrollgruppe war dies nicht der Fall $(p=0,0855)$. Zum Zeitpunkt $t 3$ ist die Sachlage unverändert, die Gruppe PF15\% ist gegenüber den beiden anderen Gruppen weiter signifikant verändert $(p<0,0001)$ und die Kontroll- und XtraBoost-Gruppe sind dies nicht $(p=0,0547)$. Zum Versuchsende ( $\mathrm{t} 4)$ sind keine statistisch erfassbaren Unterschiede zwischen den drei Versuchsgruppen mehr feststellbar. Zusammenfassend kann man feststellen, dass es im gesamten Versuchsverlauf keine signifikanten Unterschiede zwischen der Kontroll- und XtraBoostGruppe auftraten. In der PF15\%-Gruppe traten lediglich geringe Schwankungen zwischen der 4. und 12. Woche nach der Bleiche auf. Somit kann man davon ausgehen, dass die Zahnaufhellung keinen wesentlichen Einfluss auf die Veränderung des Farbparameters $b^{*}$, das heißt auf die Gelblichkeit, hatte (Tab 5.24). 
Tabelle 5.24: $p$ Werte adjustiert nach Tukey zum Vergleich der $b^{*}$-Werte innerhalb der Gruppen zu den unterschiedlichen Zeitpunkten. Statistisch signifikante Unterschiede liegen bei $p<0,05$ und sind mit * gekennzeichnet.

\begin{tabular}{|c|c|c|c|c|}
\hline Gruppe & Baseline_b & Kontrollgruppe & PF $15 \%$ & XtraBoost $38 \%$ \\
\hline Kontrollgruppe & 16.1357168 & & 0.6275 & 0.7739 \\
\hline PF $15 \%$ & 16.5903743 & 0.6275 & & 0.9691 \\
\hline XtraBoost $38 \%$ & 16.4726662 & 0.7739 & 0.9691 & \\
\hline Gruppe & $t 1 \_b$ & Kontrollgruppe & PF $15 \%$ & XtraBoost $38 \%$ \\
\hline Kontrollgruppe & 15.3096817 & & 0.7547 & 0.9616 \\
\hline PF $15 \%$ & 15.0264324 & 0.7547 & & 0.5892 \\
\hline XtraBoost $38 \%$ & 15.4151668 & 0.9616 & 0.5892 & \\
\hline Gruppe & $t 2 \_b$ & Kontrollgruppe & PF $15 \%$ & XtraBoost $38 \%$ \\
\hline Kontrollgruppe & 16.1330685 & & $<.0001^{*}$ & 0.0855 \\
\hline PF $15 \%$ & 13.0707818 & $<.0001^{*}$ & & $<.0001^{*}$ \\
\hline XtraBoost $38 \%$ & 15.2403361 & 0.0855 & $<.0001^{*}$ & \\
\hline Gruppe & $t 3 \_b$ & Kontrollgruppe & PF $15 \%$ & XtraBoost $38 \%$ \\
\hline Kontrollgruppe & 15.8535269 & & $<.0001^{*}$ & 0.0547 \\
\hline PF $15 \%$ & 13.3733435 & $<.0001^{*}$ & & $0.0014^{*}$ \\
\hline XtraBoost $38 \%$ & 14.8707512 & 0.0547 & $0.0014^{*}$ & \\
\hline Gruppe & $t 4 \_b$ & Kontrollgruppe & PF $15 \%$ & XtraBoost $38 \%$ \\
\hline Kontrollgruppe & 12.8016297 & & 0.5335 & 0.9036 \\
\hline PF $15 \%$ & 12.4128546 & 0.5335 & & 0.7975 \\
\hline XtraBoost $38 \%$ & 12.6457978 & 0.9036 & 0.7975 & \\
\hline
\end{tabular}




\subsection{Korrelation}

Der Korrelationskoeffizient nach Pearson stellt heraus, ob es eine Abhängigkeit der Farbparameter Lab* untereinander gibt. Je größer dieser Korrelationskoeffizient ist, desto stärker sind die Parameter voneinander abhängig und gegenseitig beeinflusst.

\subsubsection{Korrelation von $L^{*}$ - und $a^{*}$-Werten}

Die L*- und a*-Werte korrelieren nicht miteinander. Im gesamten Versuchszeitraum (Baseline bis $t 4)$ bleibt der Korrelationskoeffizient nach Pearson im Negativen $(-0,53631$ bis $-0,11809)$ (Tab 5.25).

Tabelle 5.25: Korrelation von $L^{*}$-und $a^{*}$-Werten: partielle Korrelationskoeffizienten nach Pearson

\begin{tabular}{|c|c|}
\hline Zeitpunkt & $\begin{array}{c}\text { Korrelationskoeffizient } \\
\text { Nach Pearson }\end{array}$ \\
\hline Baseline & $-0,37596$ \\
\hline $\mathrm{t} 1$ & $-0,11809$ \\
\hline $\mathrm{t} 2$ & $-0,53631$ \\
\hline $\mathrm{t} 3$ & $-0,49198$ \\
\hline $\mathrm{t} 4$ & $-0,40398$ \\
\hline
\end{tabular}


Auch auf den Abbildungen 5.16 bis 5.20 erkennt man keine Regelmäßigkeit, sondern nur eine willkürliche Streuung der Farbpunkte zwischen der $L^{*}$ - und $a^{*}$-Achse.

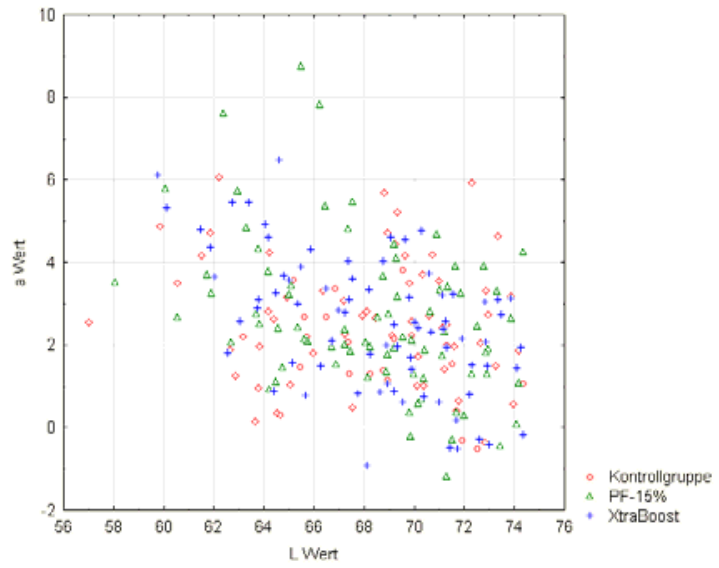

Abbildung 5.16: Korrelation $L^{*}$ - und $a^{*}$-Wert Zeitpunkt: Baseline

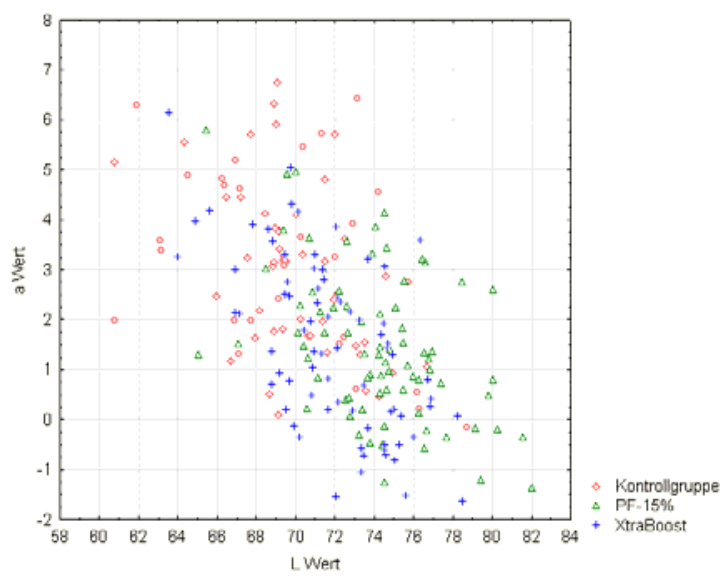

Abbildung 5.18: Korrelation $L^{*}$ - und $a^{*}$-Wert Zeitpunkt: t2

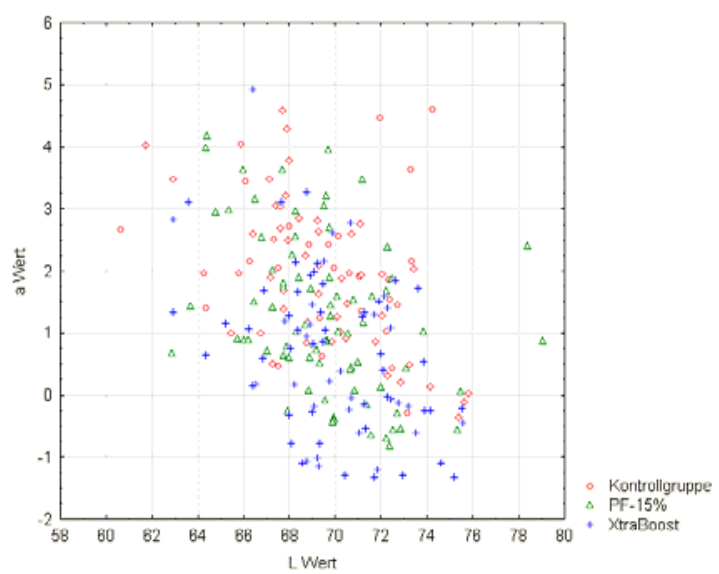

Abbildung 5.20: Korrelation $L^{*}$ - und $a^{*}$-Wert Zeitpunkt: t4

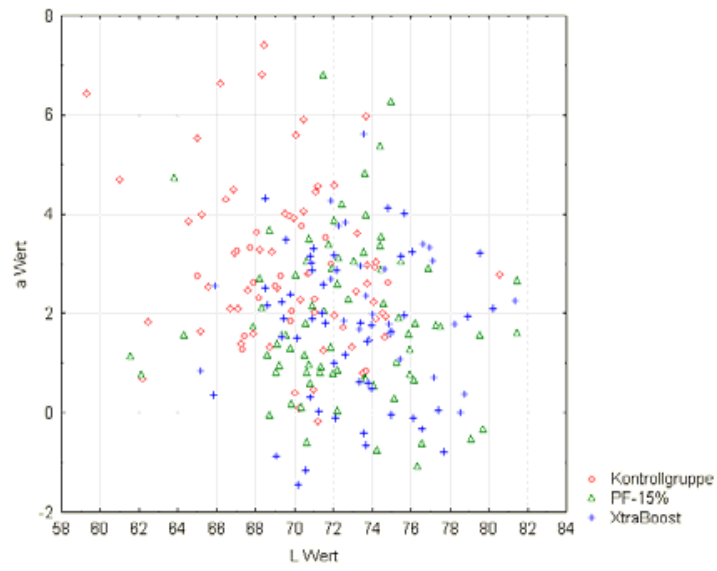

Abbildung 5.17: Korrelation $L^{*}$ - und $a^{*}$-Wert Zeitpunkt: t1

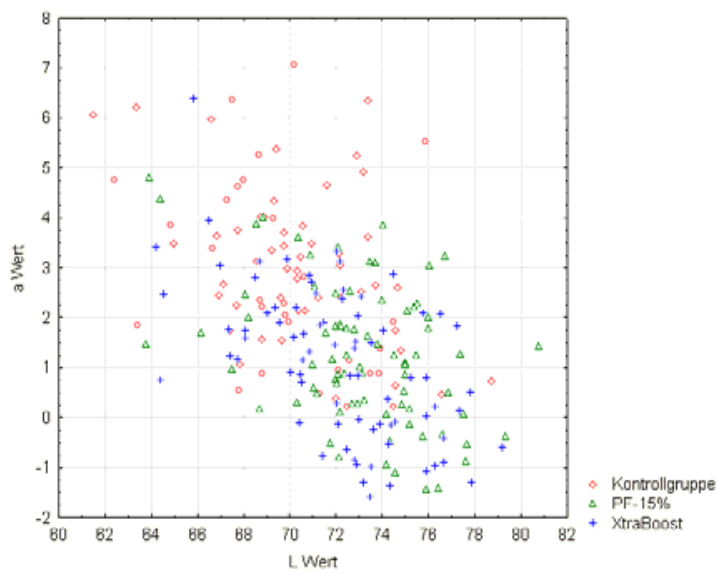

Abbildung 5.19: Korrelation $L^{*}$ - und $a^{*}$-Wert Zeitpunkt: t3 
5.7.2 Korrelation von $L^{*}$ - und $b^{*}$-Werten

Auch die $L^{*}$ - und $b^{*}$-Werte korrelieren nicht miteinander. Im gesamten Versuchsverlauf (Baseline bis t4) bleibt der Korrelationskoeffizient nach Pearson im Negativen (-0,51159 bis 0,11786) (Tab 5.26).

Tabelle 5.26: Korrelation von $L^{*}$ - und $b^{*}$-Werten: partielle Korrelationskoeffizienten nach Pearson

\begin{tabular}{|c|c|}
\hline Zeitpunkt & $\begin{array}{c}\text { Korrelationskoeffizient } \\
\text { nach Pearson }\end{array}$ \\
\hline Baseline & $-0,25517$ \\
\hline t1 & $-0,11786$ \\
\hline t2 & $-0,51159$ \\
\hline t3 & $-0,45086$ \\
\hline t4 & $-0,41660$ \\
\hline
\end{tabular}


Auch sieht man auf den Abbildungen 5.21 bis 5.25 keine Regelmäßigkeit, sondern eine willkürliche Verteilung der Farbpunkte zwischen der $L^{*}$ - und $b^{*}$-Achse.

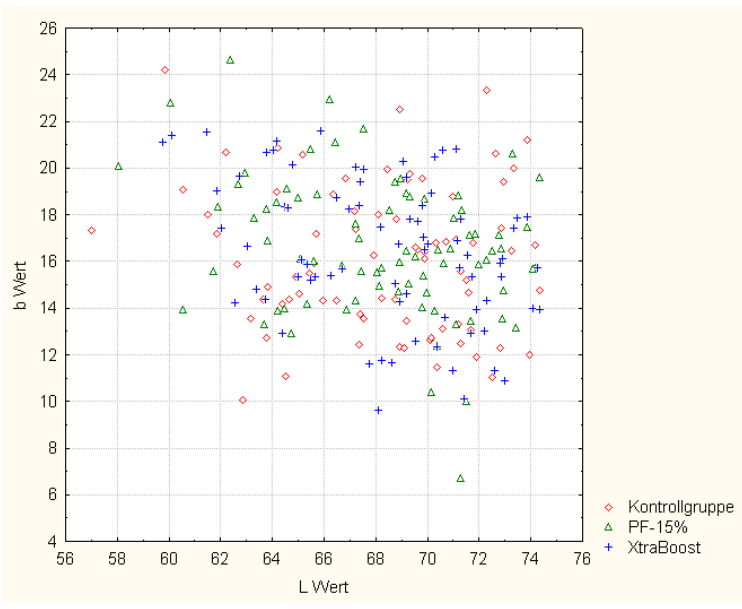

Abbildung 5.21: Korrelation $L^{*}$ - und $b^{*}$-Wert Zeitpunkt: Baseline

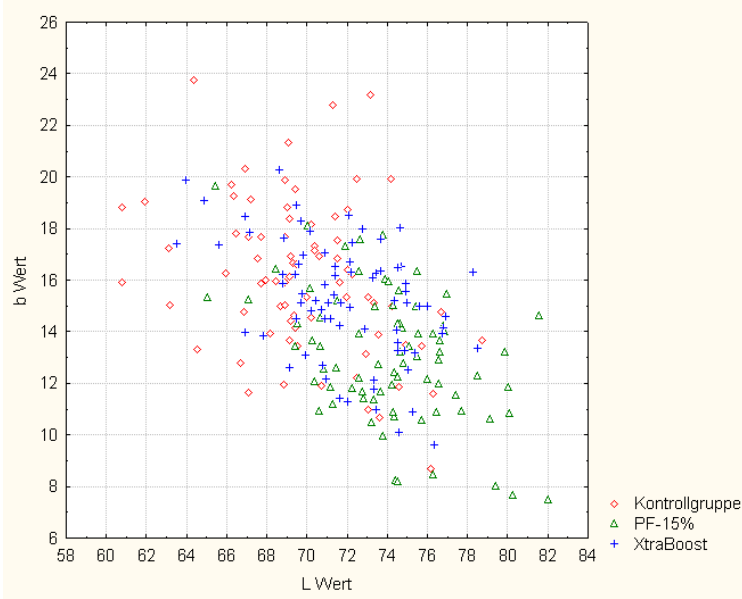

Abbildung 5.23: Korrelation $L^{*}$ - und $b^{*}$-Wert Zeitpunkt: t2

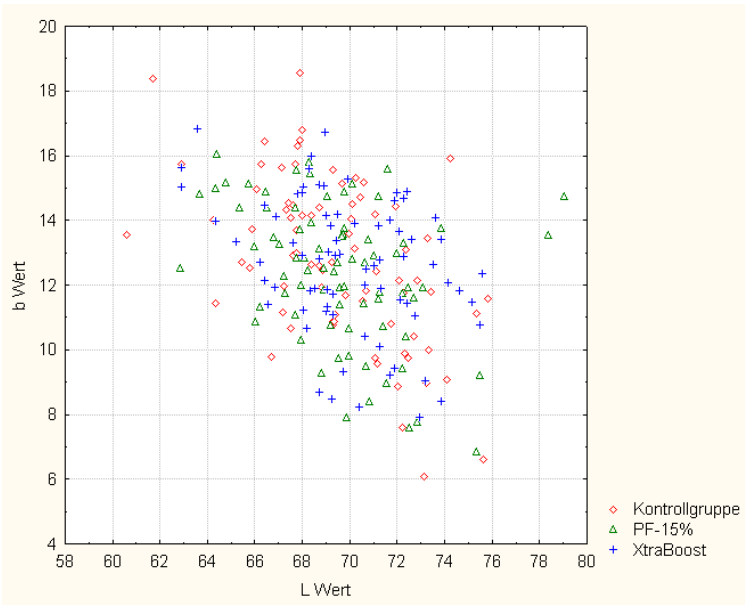

Abbildung 5.25: Korrelation $L^{*}$ - und $b^{*}$-Wert Zeitpunkt: t4

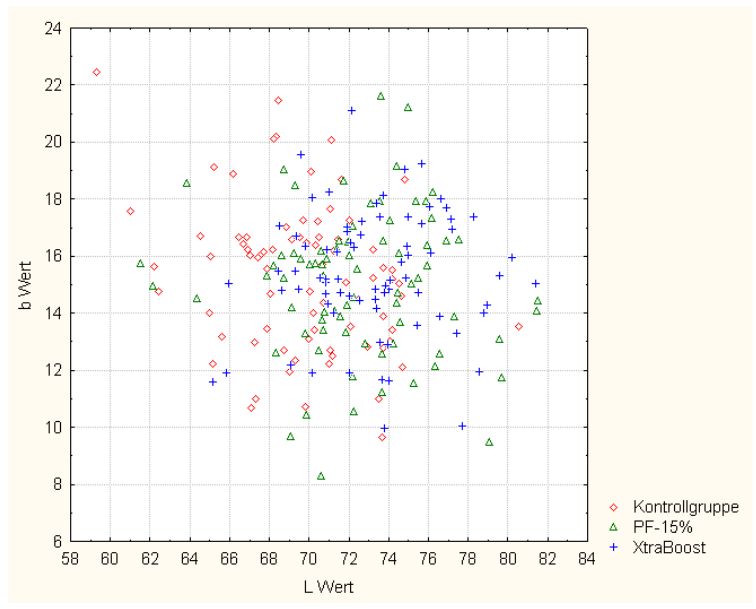

Abbildung 5.22: Korrelation $L^{*}$ - und $b^{*}$-Wert Zeitpunkt: t1

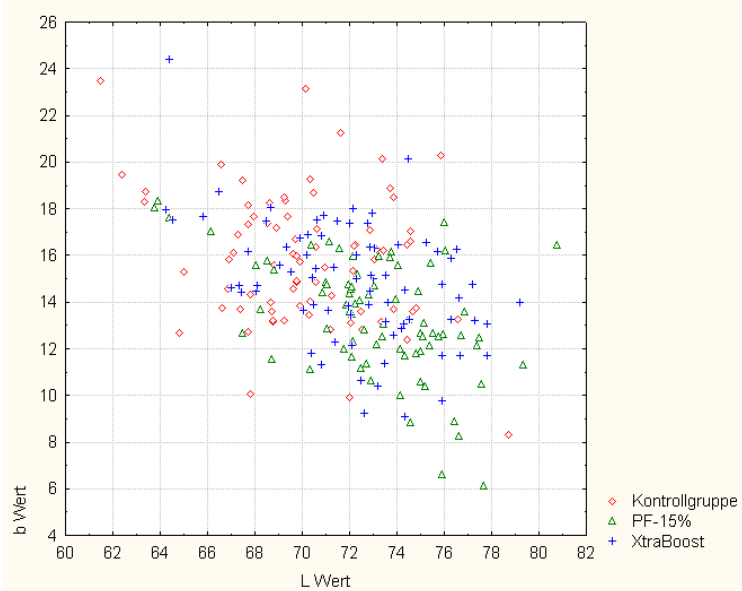

Abbildung 5.24: Korrelation $L^{*}$ - und $b^{*}$-Wert Zeitpunkt: $\mathrm{t} 3$ 
5.7.3 Korrelation von $a^{*}$ - und $b^{*}$-Werten

Die $a^{*}$ - und $b^{*}$-Werte korrelieren deutlich miteinander. Im gesamten Versuchsverlauf (Baseline bis t4) bleibt der Korrelationskoeffizient nach Pearson im Positiven (Tab 5.27).

Tabelle 5.27: Korrelation von $a^{*}$ - und $b^{*}$-Werten: partielle Korrelationskoeffizienten nach Pearson

\begin{tabular}{|c|c|}
\hline Zeitpunkt & $\begin{array}{c}\text { Korrelationskoeffizient } \\
\text { nach Pearson }\end{array}$ \\
\hline Baseline & 0,77522 \\
\hline $\mathrm{t} 1$ & 0,64057 \\
\hline $\mathrm{t} 2$ & 0,65472 \\
\hline $\mathrm{t} 3$ & 0,69242 \\
\hline $\mathrm{t} 4$ & 0,66671 \\
\hline
\end{tabular}

Im zeitlichen Verlauf der Studie ist ein Rückgang der Korrelation von 0,77522 (Baseline) bis 0,66671 (t4) zu erkennen. Dieser Rückgang lässt sich dadurch erklären, dass der $a^{*}$-Wert durch die Bleichtherapie wesentlich stärker verändert wurde als der b*-Wert (Kap. 5.3). 
Die Abhängigkeit der beiden Farbparameter $a^{*}$ und $b^{*}$ stellt sich auch graphisch in den Abbildungen 5.26 bis 5.30 dar.

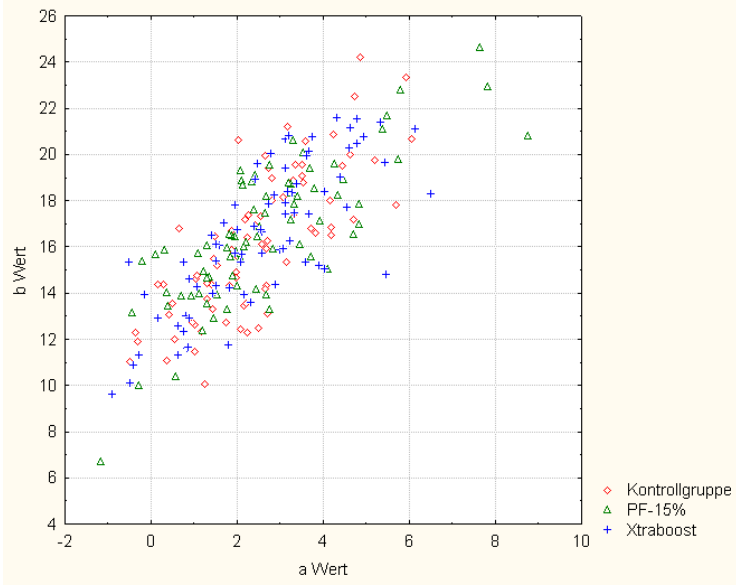

Abbildung 5.26: Korrelation $a^{*}$ - und $b^{*}$-Wert Zeitpunkt: Baseline

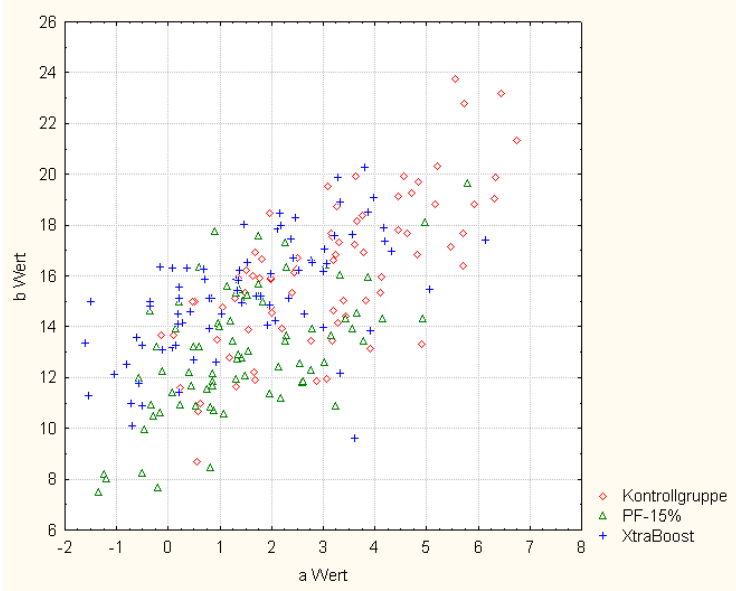

Abbildung 5.28: Korrelation $\mathrm{a}^{*}$ - und $\mathrm{b}^{*}$-Wert Zeitpunkt: t2

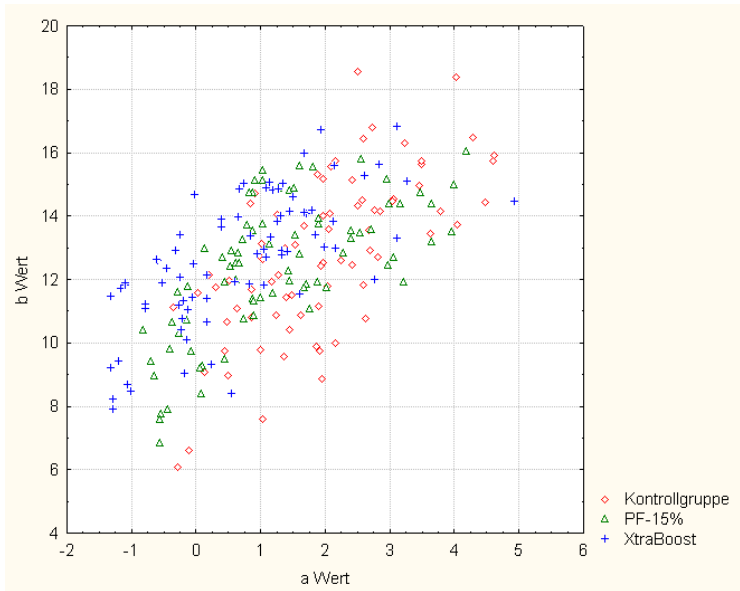

Abbildung 5.30: Korrelation $a^{*}$ - und $b^{*}$-Wert Zeitpunkt: t4

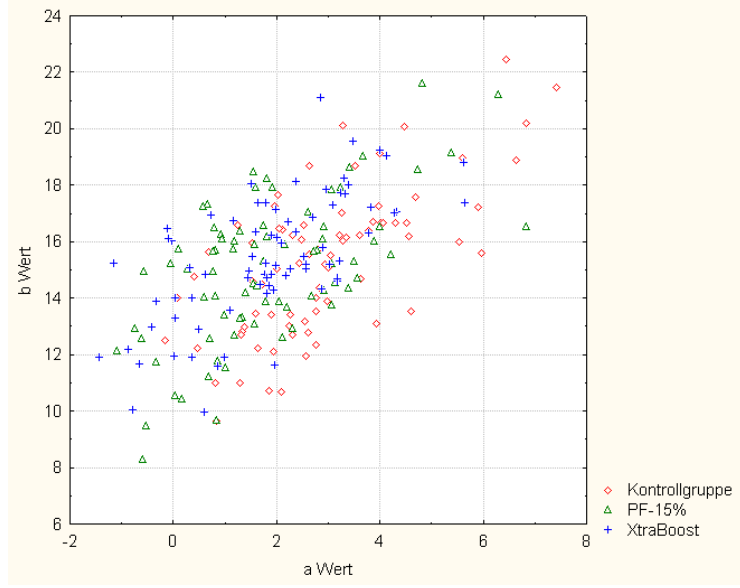

Abbildung 5.27: Korrelation $a^{*}$ - und $b^{*}$-Wert Zeitpunkt: t1

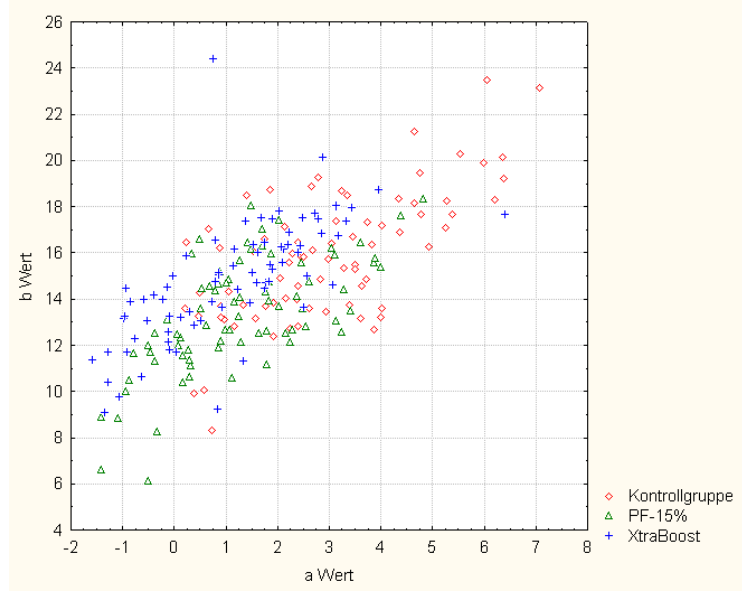

Abbildung 5.29: Korrelation $a^{*}$ - und $b^{*}$-Wert Zeitpunkt: $\mathrm{t} 3$ 


\section{Diskussion}

\subsection{Diskussion der Methode}

\subsubsection{Material - Zähne}

Ziel dieser Studie war es, mit Hilfe eines photometrisch digitalen Verfahrens festzustellen, ob es hinsichtlich der Farbstabilität einen Unterschied zwischen niedrig konzentrierten (15\%iges Carbamidperoxid) und hoch konzentrierten (35\%iges Carbamidperoxid) Bleichmitteln beziehungsweise zwischen den zwei verschiedenen Bleichmethoden Home-Bleaching und InOffice-Bleaching gibt.

In einer Vielzahl früherer Studien, die sich mit Bleichtherapien beschäftigten, wurden bovine anstatt humaner Zahnproben verwendet. Rinderzähne haben gegenüber humanen Zähnen einige Vorteile. Zum Beispiel sind sie kurzfristig und in großen Mengen verfügbar, sie haben eine größere glatte Oberfläche und meist keine kariösen Läsionen (Mellberg 1992). Boviner Schmelz weist zudem strukturelle Unterschiede, wie zum Beispiel eine höhere Porosität als humaner Schmelz, auf. Des Weiteren bestehen strukturelle Differenzen in Hinblick auf die Prismengröße, die interprismatische Substanz sowie auf das Hunter-Schräger-Muster (Whittaker 1983). Rinderzähne sind mit einer weitgehend homogenen Nahrungsaufnahme konfrontiert und somit nur wenigen äußeren Einflüssen ausgesetzt, die zu Verfärbungen führen können. Aus diesen Erkenntnissen kann man schließen, dass bovine Zahnproben strukturell den humanen zwar ähneln, sie spiegeln aber nicht die durch Lebensgewohnheiten oder das Alter hervorgerufenen Verfärbungen wider. Gerade diese Verfärbungen aber sind es vorwiegend, weshalb viele Patienten sich entscheiden, die Zähne zu bleichen.

Aus diesen Gründen wurde humanes Zahnmaterial vorgezogen. Dadurch werden die Ergebnisse dieser Studie valider, genauer und letztlich aussagekräftiger sind in Bezug auf die zahnärztliche Praxis. Die Zähne wurden im Laufe eines Jahres als extrahierte Exemplare aus verschiedenen Praxen gesammelt, genauere Angaben über Alter der Zähne, Herkunft oder Fluoridanamnese lagen somit nicht vor. Die eingesammelten Zähne wurden in ihrer ursprünglichen Form und Farbe belassen, vor Versuchsbeginn erfolgte lediglich eine professionelle Zahnreinigung zur Beseitigung von harten und weichen Belägen.

\subsubsection{Farbbestimmung}

Bei visuellen Farbbestimmungsverfahren wird die Farbwahrnehmung von vielen - auch subjektiven - Faktoren, wie zum Beispiel der Physiologie des Sehorgans (Farbenfehlsichtigkeit), der Farbe des Umfeldes (Lippenstift, Kleidung des Patienten), der Dauer der Betrachtung, 
der Form und der Oberflächengestalt des Objektes sowie des Betrachtungswinkels beeinflusst (Strub et al. 2005 b). Zudem spielen auch psychische und physische Beeinträchtigungen (Krankheit, Gemütszustand) des Betrachters eine wichtige Rolle und beeinflussen die Farbempfindung (Strub et al. 2005 b). Diese Nachteile eines visuellen Farbbestimmungsverfahrens lassen es sinnvoll erscheinen, ein standardisiertes Verfahren ohne subjektiven Faktor zu entwickeln bzw. bereitzustellen. Okubo et al. (1998), Paul et al. (2002) und Fani et al. (2007) belegten in ihren Studien, dass die Farbgebung mittels elektronischer Farbmessgeräte, wie zum Beispiel Shade Eye ${ }^{\circledR}$, eine sehr hohe Reproduzierbarkeit aufweisen $(83,3 \%$ bis $100 \%)$. Demgegenüber liegt die exakte Reproduzierbarkeit visueller Farbbestimmung nur bei 46,6\% (Paul et al 2002). Da die Reproduzierbarkeit aber ein unverzichtbares Element wissenschaftlicher Untersuchungen darstellt, ist eine visuelle Farbbestimmung bei farbvergleichenden Studien nicht zu empfehlen.

Aber auch die elektronisch technischen Analyseverfahren (zum Beispiel Shade Eye ${ }^{\circledR}$ ) sind nicht frei von Nachteilen. Ein generelles Problem des Einsatzes solcher instrumenteller Farbanalysegeräte ist, dass diese nur einen kleinen Zahnausschnitt bewerten. Somit spiegelt die ermittelte Zahnfarbe nur diesen kleinen Ausschnitt und nicht die Gesamtfarbe der Zahnoberfläche wider. Im Allgemeinen werden für die Farbprüfung homogene Strukturen mit weitgehend glatten Oberflächen benötigt. Da Zähne diesen Anforderungen nur partiell genügen, ist eine solche instrumentelle Farbbestimmung unzureichend (Haywood et al. 1994).

Neben der visuellen und instrumentellen Farbanalyse bietet die photometrische Farbanalyse seit Einführung der digitalen Fotografie eine weitere Alternative zur Bestimmung der Zahnfarbe. Bei dieser neuen Methode wird mit Hilfe einer digitalen Spiegelreflexkamera das Zahnobjekt standardisiert fotografiert. Anschließend werden die digitalen Fotos mit Hilfe eines kommerziellen Computerprogramms, hier Adobe Photoshop $\mathrm{CS}^{\circledR}$, ausgewertet (Bengel 2003). Wie in Kap. 2.5 beschrieben, erhält man bei instrumentellen Farbanalysen Lab*Werte. Im Gegensatz zum elektronischen Shade Eye ${ }^{\circledR}$ Analyseverfahren, mit dem man nur einen kleinen Ausschnitt aus der Schmelzoberfläche erfassen kann, wird bei der photometrischen Analyse mit Adobe Photoshop $\mathrm{CS}^{\circledR}$ ein Großteil der Zahnoberfläche vermessen (Bildausschnitt wird mit der Lassofunktion festgelegt) und in die Farbbestimmung mit einbezogen. Damit spiegelt die ermittelte Zahnfarbe die tatsächliche Farbe des gesamten Zahnes wider. Zusätzlich bieten digitale Fotos gegenüber den instrumentellen Farbanalyseverfahren den Vorteil, dass man neben der optischen Bildauswertung durch die Digitalisierung der Bilder gespeicherte Datensätze erhält, die man zum Beispiel weiteren statistischen Auswertungen zugrunde legen kann (Bengel 2003).

Für wissenschaftliche Studien ist die digitale photometrische Farbanalyse damit gegenüber den beiden vorgenannten Methoden im Vorteil. Sie vereinigt zwei für diese Aufgabe wesentliche Vorzüge. Eine hohe Reproduzierbarkeit in der Auswertung in Kombination mit einer 
unkomplizierten Speicherung der Ergebnisse als Datensätze für eine weitere computergestützte standardisierte Analyse (Bengel 2003, Bentley et al. 1999).

Voraussetzung für eine Vergleichsstudie ist eine exakte Standardisierung aller Parameter. Es muss gewährleistet sein, dass Einstellungen, Lichtverhältnisse, Winkel, Hintergrund und Abstand bei jedem Zahnbild exakt die gleichen Werte annehmen. Dazu wurden die Kamera und die Zahnproben in einer festen, eigens dafür hergestellten Halterung befestigt und immer im gleichen, vollständig abgedunkelten Raum fotografiert. Ein Ringblitz als einzige Lichtquelle sorgte dafür, dass die in der Mitte auf einem Metallstift fixierte Zahnprobe stets im gleichen Abstand und unter gleichem Winkel beleuchtet wurde. Zur Farbstandardisierung wurde an jedem Zahn eine Graukarte QP Card 101 (QPcardAB, Göteborg, Sweden) als Referenzfarbe für den späteren Abgleich eines Bildbearbeitungsprogramms angebracht. In Studien von Ziebolz et al. (2007) und Knösel et al. (2009) wurde diese photometrische Farbanalyse bereits erfolgreich klinisch getestet und die Standards definiert und festgelegt.

Mit Hilfe dieser festgelegten Standards ist es nun möglich, dieses neue photometrische Verfahren auch für umfangreichere Laborstudien einzusetzen. Dies ist Ziel der vorliegenden Studie, in der erstmals innovativ dieses neue photometrische Verfahren eingesetzt wurde, um in einer wissenschaftlichen Vergleichsstudie Farbänderungen zu messen. Bisher wurden noch nie in einer Laborstudie mit extrahierten Zähnen über einen langen Zeitraum mit Hilfe digitaler Fotos und unter Einsatz des Computerprogramms Photoshop ${ }^{\circledR}$ Farbveränderungen nach verschiedenen Zahnbleichungsmethoden statistisch untersucht.

\subsubsection{Lagerung}

Um eine austrocknungsbedingte Farbveränderung auszuschließen und die klinische Realsituation möglichst gut zu imitieren, wurden die Zähne zwischen den Aufnahmezeitpunkten in künstlichem Speichel gelagert. Der in dieser Studie verwendete synthetische Speichel (Klimek et al. 1982) ist dem natürlichen Speichel in seiner Zusammensetzung sehr ähnlich (Vissink et al. 1985). Durch den Zusatz von Muzin wurde der Kunstspeichel visköser und es wurde zudem eine bessere Haftung des Speichels an der Zahnoberfläche erzielt.

Da dunkle Zähne stärker durch einen Bleichvorgang aufgehellt werden als helle Zähne (Plein 2008), war es wichtig, in allen drei Versuchsgruppen (PF15\%-Gruppe, XtraBoost-Gruppe und Kontrollgruppe) gleiche Voraussetzungen hinsichtlich der Ausgangsfarbe zu gewährleisten. Dies wurde durch eine Zahnmischung erreicht, deren anfänglicher durchschnittlicher $L^{*}$ Wert gleich war. 


\subsubsection{Bleichtherapie}

Ziel dieser Studie war nicht das Bleichresultat des Bleichmittels selbst, sondern die Langzeitstabilität der Bleichtherapie. Daher war es unbedingt notwendig, in beiden Versuchsgruppen PF15\% und XtraBoost ein vergleichbares Bleichergebnis zu erzielen. Des Weiteren musste in beiden Gruppen eine deutliche Farbänderung erzielt werden, da nur so das zu erwartende Abklingen der Aufhellung mit der nötigen Genauigkeit registriert werden konnte. Somit war es nicht möglich, nach den Herstellerangaben zu bleichen, da die zwei verschieden Verfahren, Home-Bleaching und In-Office-Bleaching, unterschiedliche Konzentrationen von Carbamidperoxid aufweisen.

Auschill et al. (2005) stellten in ihrer Untersuchung fest, dass man das gleiche Bleichergebnis erhält, wenn man Opalescence PF10\% ${ }^{\circledR} 7$ mal 8 Stunden und Opalescence XtraBoost ${ }^{\circledR} 3$ mal 15 Minuten einwirken lässt. Da in dieser Studie 15\%ige und nicht wie bei Auschill et al. (2005) 10\%ige Carbamidperoxidkonzentration im Opalescence PF enthalten war, wurde der Bleichvorgang auf 5 Zyklen reduziert.Das Home-Bleaching-Verfahren wird in der Regel mit Hilfe von individuell angepassten Kunststoffschienen durchgeführt. Aufgrund der Durchführung der Bleichtherapie an extrahierten Zähnen wurde das Schienenbleichen modifiziert durch Anwendung von Frasaco Stripkronen ${ }^{\circledR}$ (Frasaco $\mathrm{GmbH}$, Tettnang, Germany) und so praxisnah nachgestellt. Der Einsatz solcher Schienen beziehungsweise Stripkronen ist unabdingbar, da sie ein Reservoir für das Bleichgel am Zahn schaffen. Somit verbleibt das Bleichgel länger und mit einer gewissen Schichtstärke an der Zahnoberfläche, was Einfluss auf die Wirksamkeit der Bleichtherapie hat (Maties et al. 2002). Durch diese Maßnahme soll gewährleistet werden, dass die Bleichergebnisse der vorliegenden in-vitro-Untersuchung auch auf in-vivo-Fälle übertragbar und interpretierbar sind.

Das Bleichmittel Opalescence PF $15 \%{ }^{\circledR}$ (Ultradent, South Jordan, USA) wurde dabei in die Frasaco Sripkronen ${ }^{\circledR}$ appliziert und anschließend über die Zahnproben gestülpt. Analog zu den Verhältnissen im Mund bei der Home-Bleaching-Technik kamen die Zahnproben anschließend über den gesamten Bleichzeitraum von 8 Stunden in die künstliche Speichellösung.

Beim In-Office-Verfahren erfolgt das Bleichen am Patienten unter weitgehender Trockenlegung mittels Kofferdam. Entsprechend gestaltete sich der Bleichvorgang in der vorliegenden in-vitro-Untersuchung einfach. Die Zähne wurden vor dem Bleichvorgang lediglich getrocknet, mit dem Bleichmittel bestrichen und anschließend für 15 Minuten außerhalb der künstlichen Speichellösung gelagert. Die Kontrollgruppe blieb unbehandelt. 


\subsubsection{Abschließende Bewertung}

Die Auswertung der Studie hat gezeigt, dass das methodische Vorgehen von der beschriebenen Bleichtherapie der Zahnproben, der monatelangen Lagerung in Speichelersatz bis hin zur photometrischen Auswertung und statistischen Beurteilung geeignet ist, Bleicheffekte zu erzeugen, zu erfassen, zu untersuchen und auszuwerten.

Besonderer wird dabei auch auf den langen Untersuchungszeitraum von 6 Monaten hingewiesen, der hinsichtlich einer Vergleichbarkeit und Stabilität aller Parameter eine besondere Herausforderung darstellte, die nur durch einen soliden und robusten photometrischen Aufbau realisiert werden konnte. Dieser hat sich in der halbjährigen Praxis bewährt und war Grundlage verlässlicher Daten.

Dabei wurde in allen Untersuchungsphasen stets darauf geachtet, dass das in-vitro-Modell durch Nachbildung praxisnaher beziehungsweise klinischer Gegebenheiten auch Rückschlüsse auf in vivo zulässt. Dazu gehörte der Einsatz menschlicher Zähne ebenso wie deren korrekte Auswahl und ein einwandfreies Randomisierungsverfahren zur anfänglichen Zahnmischung. Zudem wurden die Zahnproben über den gesamten Versuchszeitraum von 6 Monaten regelmäßig wechselnden Lichteinflüssen ausgesetzt, um der in-vivo-Realsituation zu genügen.

Die Durchführung der Studie und deren Auswertung haben letztlich gezeigt, dass das angewandte Analyseverfahren stringent durchdacht und methodisch solide aufgebaut ist. Somit können die gewonnenen Ergebnisse als gesichert und reproduzierbar eingestuft und eine Übertragung der Resultate auf die in-vivo-Realsituation als gesichert angesehen werden.

\subsection{Diskussion der Ergebnisse}

Zentraler Inhalt dieser Studie ist die methodische Untersuchung sowie die Bewertung und Dokumentation des Verlaufs der Farbveränderungen von extrahierten Zähnen nach Aufhellung mit zwei verschiedenen carbamidperoxidhaltigen Bleichmitteln.

Dazu wurden die Bleichtherapien Home-Bleaching mit Opalescence PF15\% ${ }^{\circledR}$ und In-OfficeBleaching mit Opalescence XtraBoost ${ }^{\circledR}$ eingesetzt. Die Bleichwirkungen wurden zusammen mit einer unbehandelten Kontrollgruppe in bestimmten zeitlichen Abständen über einen Zeitraum von 6 Monaten photometrisch bestimmt und anschließend statistisch ausgewertet. $\mathrm{Ne}-$ ben dem Helligkeitsparameter $L^{*}$ gibt das photometrisch Verfahren einen a*-Wert als korrespondierenden Wert zur Rötlichkeit beziehungsweise einen $b^{*}$-Wert als korrespondierenden Wert zur Gelblichkeit der Zahnoberfläche wieder. Zusammen mit dem L*-Wert sind sie maßgebend für das farbliche Zahnbild. 


\subsubsection{L*-Wert}

Die Bleichtherapie führte sowohl in der PF15\% als auch in der XtraBoost-Gruppe zu einer signifikanten Änderung des Helligkeitswertes L* (Signifikanzniveau größer als 99,99\%) und somit zu einer deutlichen Aufhellung bei den eingesetzten Zahnproben. Vor allem die Veränderung der $\Delta L^{*}$ zeigt eindeutig, dass eine signifikante Bleichwirkung eingetreten ist $\left[\Delta L^{*}\right.$ $($ PF15\% $)=4,33$ und $\Delta L^{*}($ XtraBoost $\left.)=4,88\right]($ Tab 5.1 Baseline zu t1 $)$.

Zentraler Inhalt dieser Vergleichsuntersuchung war die Dokumentation des Verlaufs der Farbveränderung. Somit war es Ziel der Bleichtherapie, in beiden Gruppen ein vergleichbares Bleichergebnis als Ausgangswert zu erhalten. Die Auswertung nach der Bleichtherapie zeigte keinen signifikanten Unterschied im L*-Wert. Somit wurde in beiden Bleichgruppen der gleiche Bleicheffekt erzielt.

Grund zur Diskussion bietet eine auffällige weitere Aufhellung der Zahnproben in der PF15\%-Gruppe bis 4 Wochen nach der Bleichtherapie auch ohne weitere Bleichgelapplikation (Tab 5.1). Eine mögliche Erklärung dieser Nachhellung liefert eine Studie von Rosentritt et al. aus dem Jahr 2003. Hierin wurde nachgewiesen, dass der bei der Bleichtherapie eingelagerte Sauerstoff noch für mehrere Wochen aktiv ist, was zu nachträglichen Aufhellungen führen kann. Dass diese Nachbleichung in der vorliegenden Studie nur in der PF15\%Gruppe auftrat, könnte mit der (oben beschriebenen) intensiveren Schienenbleichtherapie und der damit verbundenen langen Einwirkzeit des Carbamidperoxidgels in Zusammenhang stehen. Bei der angewandten Bleachingzeit von jeweils 8 Stunden an 5 hintereinander liegenden Tagen ist davon auszugehen, dass vermehrt Sauerstoff freigesetzt und in die Zahnhartsubstanz eingelagert wurde. Im Vergleich dazu ist bei der In-Office-Methode die Einwirkzeit des Bleichgels Opalescence XtraBoost ${ }^{\circledR}$ von 3 mal 15 Minuten deutlich kürzer.

Durch diese Nachhellung ist zu erklären, dass das Bleichergebnis in der PF15\%-Gruppe in den Messungen nach 4 beziehungsweise 12 Wochen deutlich über der der XtraBoostGruppe liegt (Abb 5.5). Der Bleicheffekt des Home-Bleaching-Präparats Opalescence PF15\% ${ }^{\circledR}$ wirkt in diesem Zeitraum nachhaltig stabiler. Dies deckt sich auch mit einer Studie von Zenkonis et al. (2003). Darin gaben $84 \%$ der Testpersonen an, dass sie das HomeBleaching für effektiver halten und $16 \%$ konnten keinen Unterschied zwischen den beiden Bleichungen feststellen. Kein Proband favorisierte die In-Office-Methode.

Zahnaufhellungen sind nicht unbegrenzt stabil, jede Bleichung dunkelt über einen längeren Zeitraum nach. Dies konnte auch in der vorliegenden Studie deutlich anhand der $L^{*}$-Werte nach 6 Monaten festgestellt werden (Tab 5.1, Abb 5.5).

Interessant ist, dass das Bleichergebnis des Home-Bleaching-Präparats Opalescence PF15\% ${ }^{\circledR}$ nach 6 Monaten Versuchsverlauf nicht mehr besser abschneidet als das des Opa- 
lescence $X$ traBoost $^{\circledR}$-Präparates. Die Werte lagen sogar geringfügig darunter $\left[L^{*}(P F 15 \%)=\right.$ 69,55 beziehungsweise $L^{*}($ XtraBoost $\left.)=69,91\right]$.

Die Aufhellung der Zähne klingt im Verlauf der Studie deutlich ab. Die L*-Werte der Bleichgruppen unterschieden sich am Ende der Studie statistisch nicht mehr signifikant von denen der Kontrollgruppe (Tab 5.3).

Viele bisherige Untersuchungen zur Bleichwirkung wurden nur über kürzere Zeiträume erstellt (Gerlach und Zhou 2002: 2 Wochen, Knösel et al. 2009: 2 Tage, Paul et al. 2002: 8 Tage, Zenkonis et al. 2003: 12 Wochen, Ziebolz et al. 2007: 2 Wochen). Man kann davon ausgehen, dass die Bleichwirkung beider Verfahren nach 6 Monaten soweit abgeklungen ist, dass eine Unterscheidung untereinander und auch zur Kontrollgruppe der ungebleichten Zähne nicht mehr gesichert möglich ist. Dies kann als zeitlicher Anhaltspunkt für weitere Bleichstudien dienen.

\subsection{2 $a^{*}-$ Wert}

Der $a^{*}$-Wert nahm nach der Bleichtherapie signifikant ab. Diese Abnahme setzte sich über den gesamten Untersuchungszeitraum kontinuierlich fort (Abb 5.10). Dieses nachwirkende Ausbleichen der Rötlichkeit behandelter Zähne kann ebenfalls durch den nachhaltig wirksamen Sauerstoff, der durch die Bleichung in die Zahnhatsubstanz eingelagert wurde, erklärt werden (Rosentritt et al. 2003). Alle $a^{*}$-Werte der Bleichgruppen liegen während des gesamten Untersuchungszeitraumes beständig unterhalb der Werte der Kontrollgruppe. Durch die fehlende Annäherung der $a^{*}$-Werte an die Kontrollgruppe kann man von einem stabilen Bleichergebnis ausgehen. Jedenfalls kann dieses Ergebnis dahingehend interpretiert werden, dass im Gegensatz zu den oben beschriebenen Helligkeitswerten L* die Verschiebungen in der Rötlichkeit - zumindest in diesem Untersuchungszeitraum von 6 Monaten - nicht reversibel, sondern dauerhaft angelegt sind.

\subsection{3 $b^{*}$-Wert}

Die $b^{*}$-Werte, das heißt die Gelblichkeit, zeigten ein eher indifferentes Verhalten (Abb 5.15). Auffallend ist, dass es während des gesamten Untersuchungszeitraumes keinen statistisch signifikanten Unterschied zwischen der XtraBoost- und der ungebleichten Kontrollgruppe gab. Nach der Bleichtherapie sind zunächst in allen drei Gruppen die b*-Werte konstant. Nach 4 Wochen fällt der b*-Wert der PF15\%-Gruppe (Verblassung des Gelbwertes) und bewegt sich weiterhin unterhalb der anderen beiden Gruppen. Nach 6 Monaten, das heißt bei Versuchsende, haben sich die $b^{*}$-Werte wieder angenähert, so dass ein Unterschied zwischen beiden behandelten Gruppen und der unbehandelten Kontrollgruppe statistisch nicht 
mehr festzustellen ist. Die insgesamt geringen Abweichungen bei den $b^{*}$-Werten im Vergleich zur unbehandelten Kontrollgruppe lassen darauf schließen, dass die Applikation sowohl mit hochkonzentrierten Carbamidperoxidpräparaten (Opalescence XtraBoost ${ }^{\circledR}$ ) wie auch mit niedrig konzentrierten Präparaten (Opalescence PF15\% ${ }^{\circledR}$ ) keinen nennenswerten Einfluss auf die Gelblichkeit der Zahnoberfläche haben.

Auffallend ist der signifikante Abfall der $a^{*}$-Werte und $b^{*}$-Werte bei der XtraBoost-Gruppe ( $a^{*}$ Wert von 1,13 auf 0,68 beziehungsweise $b^{*}$-Wert von 14,87 auf 12,65) und der Kontrollgruppe ( $a^{*}$-Wert von 2,96 auf 1,95 beziehungsweise $b^{*}$-Wert von 15,85 auf 12,8) in den letzen 3 Monaten der Untersuchung (Abb 5.10, Abb 5.15). Es wurden eine Reihe von beeinflussenden Faktoren diskutiert (Lagerung / Lichteinfluss etc), die sich aber allesamt als nicht stichhaltig erwiesen, da sich diese Veränderungen auch auf die PF15\%-Gruppe und auf die Helligkeitswerte $L^{*}$ hätten auswirken müssen. Insbesondere kann demzufolge auch ein Apparate- oder Messfehler nicht ausgeschlossen werden.

\subsubsection{Korrelation}

Abschließend sei noch erwähnt, dass eine Korrelationsstatistik gezeigt hat, dass die Farbparameter $a^{*}$ und $b^{*} z u$ jedem Zeitpunkt der Untersuchung voneinander abhängig sind (Abb 5.26 bis Abb 5.30, Tab 5.12). Im Gegensatz dazu entwickeln sich die Helligkeitswerte $L^{*}$ unabhängig, das heißt sie korrelieren nicht mit den Farbwerten $a^{*}$ und $b^{*}$ (Abb 5.16 bis Abb 5.25, Tab 5.10, Tab 5.11).

\subsection{Schlussfolgerung}

In der vorliegenden Studie konnte mit Hilfe eines photometrischen Verfahrens gezeigt werden, dass Zahnbleichtherapien sowohl mit hoch als auch mit niedrig konzentriertem Carbamidperoxid in ihrer Bleichwirkung zwar unmittelbare Erfolge erzielen, aber nicht dauerhaft über einen Zeitraum von 6 Monaten stabil sind.

Über den Untersuchungszeitraum von 6 Monaten war in beiden Bleichgruppen ein Rückgang der Weißaufhellung ( $L^{*}$-Wert) bis hin zum Ausgangswert erkennbar. Lediglich die Aufhellung des rötlichen Farbton ( $a^{*}$-Wert) der Zähne blieb bis zum Versuchende nachhaltig stabil.

Der Einfluss der Bleichtherapie auf den gelblichen Farbton ( $b^{*}$-Wert) war nicht deutlich erkennbar. Durch die hoch konzentrierten Carbamidperoxid-Produkte wurde er nicht nachweisbar gesenkt und bei niedrig konzentrierter Applikation war nur ein geringer Einfluss erkennbar. 
Zwischen beiden Bleichprodukten konnte kein entscheidender Unterschied festgestellt werden. Daher kann aufgrund der Bleichwirkung und Nachhaltigkeit der Präparate keinem ein Vorzug gegeben werden.

Abschließend sei erwähnt, dass eine Validierung der vorliegenden Daten und des Versuchsaufbaus durch klinische Studien erforderlich ist. 


\section{Zusammenfassung}

Ziel dieser Studie war es, die Wirksamkeit von Zahnbleichungen über einen längeren Zeitraum zu untersuchen. Insbesondere ging es darum festzustellen, wie sich die Bleichwirkungen von niedrig konzentrierten (15\%iges Carbamidperoxid) und hoch konzentrierten (35\%iges Carbamidperoxid) Bleichmitteln zeitlich verhalten. Die unterschiedlichen Konzentrationen der Bleichmittel sind Grundlage der beiden verschiedenen gängigen Bleichmethoden Home-Bleaching (Opalescence PF $15 \%{ }^{\circledR}$ ) und In-Office-Bleaching (Opalescence XtraBoost $\left.{ }^{\circledR}\right)$. Die Studie wurde durch eine weitgehend praxis- beziehungsweise realitätsnahe Durchführung auf einen Langzeitvergleich der Bleichwirkung dieser beiden Bleichverfahren angelegt. Um eine gesicherte Übertragbarkeit der Resultate auf die Praxis zu gewährleisten, wurden ausschließlich humane Zahnproben verwendet. Um ein Höchstmaß an Objektivität zu gewinnen, wurde zur Farbanalyse ein auf digitaler Fotografie basierendes photometrisches Messverfahren eingesetzt. Die Erprobung dieser modernen Farbbestimmung im Rahmen einer Langzeitstudie war ein weiterer Aspekt dieser Studie auch im Hinblick auf die Praxistauglichkeit.

Zur Datengewinnung wurden 231 extrahierte menschliche Front- und Eckzähne (Zahnfarbe A3 und dunkler) mit Hilfe von digitalen Fotos und dem kommerziellen Computerprogramm Adobe Photoshop $\mathrm{CS}^{\circledR}$ mehrfach innerhalb der Studiendauer farblich ausgewertet. Es wurde die Zahnfarbe nach dem Lab*-Farbschema (CIELab*-Wert; L*: Helligkeitswert, a*: rot/grünWert, $b^{*}$ : gelb/blau-Wert) bestimmt. Anhand des anfänglich festgestellten L*-Wertes (Helligkeit) wurden die 231 Zähne in die drei Versuchsgruppen zu je 77 Zähnen gleichmäßig aufgeteilt (Kontrollgruppe, PF15\% und XtraBoost). Anschließend wurden die Zahnproben in modifizierter Form in Anlehnung an eine Studie von Auschill et al. aus dem Jahr 2005 praxisnah gebleicht. Die Gruppe PF15\% (Home-Bleaching) wurde mit Opalesence PF15\% ${ }^{\circledR}$ an 5 aufeinander folgenden Tagen für jeweils 8 Stunden und die Gruppe XtraBoost (In-OfficeBleaching) wurde mit Opalesence $\mathrm{XtraBoost}^{\circledR}$ an drei aufeinander folgenden Tagen für jeweils fünfzehn Minuten gebleicht. Die Kontrollgruppe verblieb unbehandelt.

3 Tage nach den Bleichtherapien sowie nach 30, 90 und 180 Tagen wurde von jedem Zahn eine komplette Farbanlyse erstellt, deren ermittelte Lab*-Werte mit Hilfe einer Statistik Rückschlüsse auf fortschreitende signifikante Veränderungen erlauben sollten.

Um der Realsituation weitestgehend gerecht zu werden, lagerten die Zähne während des Versuchszeitraums in künstlicher Speichellösung und wurden abwechselnd bei $37^{\circ} \mathrm{C}$ jeweils zwei Wochen im Tageslicht beziehungsweise abgedunkelt aufbewahrt.

Die Auswertung nach 3 Tagen ergab, dass beide Bleichmittel zu nachweislich guten Bleichergebnissen führen mit signifikanten Aufhellungen in den Helligkeits- und Rötlichkeitswerten. 
Bei den Gelblichkeitswerten konnten kaum Aufhellungen festgestellt werden. Die Kontrollgruppe blieb erwartungsgemäß unverändert.

Auch nach 30 Tagen und 90 Tagen ergaben die Messungen, dass beide Bleichungen nachhaltig wirkten. Erst nach 180 Tagen ließ der Bleicherfolg nach. Während in der PF15\%Gruppe der Ausgangszustand nahezu wieder erreicht wurde, zeigte die Gruppe XtraBoost noch eine verbliebene Restaufhellung mit einem geringen signifikanten Unterschied zur Baseline. Demgegenüber blieben die Rötlichkeitswerte $\left(a^{*}\right)$ in beiden Bleichgruppen weiterhin signifikant verringert, was auf eine nachhaltige Bleichung hindeutet. Die Gelblichkeitswerte $\left(b^{*}\right)$ zeigten nur geringe Veränderungen, zum Teil auch indifferentes Verhalten, was keine Rückschlüsse auf eine Wirkung durch die Bleichung zulässt.

In der vorliegenden Studie konnte somit gezeigt werden, dass sowohl hoch- als auch niedrigkonzentrierte Carbamidperoxidgele zu einer signifikanten Zahnaufhellung führen, diese jedoch in ihrer vollständigen Bleichwirkung nicht dauerhaft stabil sind. Nach 6 Monaten sind insbesondere die Helligkeitswerte fast wieder auf das Ausgangsniveau zurückgefallen. Am ehesten ist eine nachhaltige Aufhellung in der Rötlichkeit der Zähne feststellbar.

Ein gravierender Unterschied zwischen den beiden Methoden Home-Bleaching und InOffice-Bleaching bezüglich der Farbstabilität war ebenfalls nicht feststellbar.

Die photometrische Methode zur Farbanalyse hat sich - trotz des hohen apparativen Aufwands - für wissenschaftliche Studien bestens bewährt. Sie lieferte objektive, reproduzierbare und verlässliche Datensätze, die mit anderen Farbanalysemethoden so nicht möglich gewesen wären. Letztlich erlaubte erst diese große Datenmenge eine stochastische Auswertung und damit eine statistische Absicherung der gewonnenen Ergebnisse. 


\section{Literaturverzeichnis}

Attin T (1998):

Sicherheit und Anwendung von carbamidperoxidhaltigen Gelen bei Bleichtherapien.

Dtsch Zahnärztl Z $\underline{53}, 11-16$

\section{Attin T:}

Praxiscoach Bleaching - Das Handbuch für erfolgreiches Bleaching in der Zahnarztpraxis.

Schlütersche Verlagsgesellschaft, Hannover 2002

(ISBN: 3935802021)

Attin T, Kielbassa AM (1995):

Die Bleichbehandlung - ein fester Bestandteil ästhetischer Zahnheilkunde.

Zahnärztl Mitt $\underline{85}$, 2674-2681

\section{Attin T, Kielbassa AM, Schanenberg M, Hellwig E (1997):}

Effect of fluoride treatment on remineralisation of bleached enamel.

J Oral Rehabil 24, 282-86

\section{Attin T, Paque F, Ajam F, Lennon AM (2003):}

Review of the current status of tooth whitening with the walking bleach technique.

Int Endod J 36, 313-329

Attin T, Hannig C, Wiegand A, Attin R (2004):

Effects of bleaching on restorative materials and restorations - a systemativ review.

Dent Mater $\underline{20}, 852-61$

Auschill TM, Hellwig E, Schmidale S, Sculean A, Arweiler NB (2005):

Efficacy, side-effects and Patients` Acceptance of Different Bleaching Techniques.

Oper Dent $\underline{30-2}, 156-163$

\section{Baik JW, Rueggeberg FA, Liewehr FR (2001)}

Effect of light-enhanced bleaching on in vitro surface and intrapulpal temperature rice.

J Esthet Restor Dent 13, 370-378

Bailey SJ, Swift EJ Jr (1992):

Effects of home bleaching products on composite resins. 
Quintessence Int $\underline{23}, 489-94$

\section{Barnes DM, Kihn PW, Romberg E, George D, DePaola L, Medina E (1998):}

Clinical evaluation of a new $10 \%$ carbamid peroxide tooth-whitening agent.

Compend Contin Educ Dent $\underline{19}, 968$

\section{Baumann MA, Schifferdecker B (1994):}

Farbbestimmung bei Dentalkeramiken.

Schweiz Monatsschr Zahnmed 104, 423-429

\section{Ben-Amar A, Liberman R, Gorl C, Bernstein Y (1995):}

Effect of mouthguard bleaching on enamel surface.

Am J Dent $\underline{8}, 29-32$

\section{Bengel WM (2003):}

Digital Photography and the Assessment of Therapeutic Results after Bleaching Procedures.

J Esthet Restor Dent $\underline{15}, 21-32$

\section{Bentley C, Leonard RH, Nelson CF, Bentley SA (1999):}

Quantitation of vital bleaching by computer analysis of photographic images.

J Am Dent Assoc 130, 809-816

\section{Burgmaier GM, Schulze IM, Attin T (2002):}

Fluorid uptake and development of artificial erosions in bleached and fluoridated enamel in vitro.

J Oral Rehabil 29, 799-804

\section{Butler WT:}

Dentin collagen: chemical structure and role in mineralization.

In: Linde A: Dentin and Dentionogenesis. Vol. II. CRC Press, Boca Raton 1984, 34

\section{Christensen GJ (1989):}

Tooth bleaching, home use products.

Clin Res Assoc Newsletter 13, 1-2

\section{Christensen GJ (1991):}

Home-use bleaching survey. 
Clin Res Assoc Newsletter 15, 2

\section{Cooper JS, Bokmeyer TJ, Bowles WH (1992):}

Penetration of the pulp chamber by carbamide peroxide bleaching agents.

J Endod 18, 315-17

\section{Culpepper WD (1970):}

A comparative study of shade matching procedures.

J Prosthet Dent 24, 166-167

\section{DGZMK (2000):}

Aktuelle Stellungnahme der DGZMK/DGZ zum Bleichen von verfärbten Zähnen Stand 2000 http://www.dgzmk.de/zahnaerzte/wissenschaft-

forschung/stellungnahmen/details/document/das-bleichen-verfaerbter-zaehne.html

\section{Duschner H (2002):}

Zahnaufhellung mit Peroxiden.

Zahnärztl Mitt 13, 42-49

\section{Erikson HM, Nordbo H (1978):}

Extrinsic discoloration of teeth.

J Clin Periodontol $\underline{5}, 229-236$

\section{Ernst CP, Briseno B, Hickel R (1995):}

Bleichbehandlung von vitalen und avitalen Zähnen.

Phillip J 12, 229-236

Fani G, Vichi A, Davidson CL (2007):

Spectrophotometric and visual shade measurements of human teeth using three shade guides.

Am J Dent $20(3), 142-46$

\section{Feiglin B (1987):}

6-year recall study of chemically bleaches teeth.

Oral Surg Oral Med Oral Pathol $\underline{63}, 610-13$ 


\section{Feinman RA (1994):}

Bleaching vital teeth.

Curr Opin Cosmet Dent 2, 23-29

Feinman RA, Goldstein RE, Garber DA:

Bleaching teeth.

Quintessence Verlag, Chicago 1987

(ISBN: 0867151838)

\section{Feinman RA, Madray G, Yarborough D (1991):}

Chemical, optical and physiologig mechanisms of bleaching products: a reciew.

Pract Periodontics Aesthet Dent $\underline{3}, 32-36$

\section{Freidman S, Rotstein I, Libfeld H, Stabholz A, Heling I (1988):}

Incidence of external root resorption and esthetic results in 58 bleaches pulpless teeth.

Endod Dent Traumatol 4 , 23-26

\section{GarberoglioR, Brännström M (1976):}

Scanning electron microscopic investigation of human dentinal tubules.

Arch Oral Biol 21, 355-362

\section{Gerlach RW, Zhou X (2001):}

Vital bleaching with whitening strips: summary of clinical research on effectiveness and tolerability.

J Contemp Dent Pract 2, 1 1-16

\section{Gerlach RW, Zhou X (2002):}

Comparative clinical efficacy of two professional bleaching systems.

Compend Contin Educ Dent $\underline{23}$, 35-41

\section{Geurtens W, Vernieks AA (1986):}

Das Bleichen verfärbter Zähne.

Zahnärztl Welt $\underline{95}, 54-56$

\section{Glockner K, Jeglitsch F, Städler P, Ebelseder K (1995):}

Die Zunahme der Sprödigkeit von Dentin beim „internal bleaching“. 


\section{Glockner K, Ebelseder K, Städler P (1997):}

Das Bleichen von verfärbten Frontzähnen.

Dtsch Zahnärztl Z 르, 311-317

\section{Goldstein RE, Garber DA:}

Complete dental bleaching.

Quintessence Publishing Co Ltd, Chicago 1995

(ISBN: 0867152907)

Grehn J, Krause J:

Metzler Physik

3. Aufl., S.373, Schroedel Verlag, Hannover 1998

(ISBN: 3507107007)

Grote M, Kuhaupt H, Wölbern N, Zimmerschied F:

Impulse Physik 2

Klett Verlag, Stuttgart 2008, 144

(ISBN: 3127724752)

\section{Guldener PHA, Langeland K:}

Endodontologie

2. Aufl., Thieme Verlag, Stuttgart 1987

(ISBN: 3136100034)

\section{Hassink C:}

Verschiedene Methoden des Bleichens vitaler Zähne: Ein elektronenmikroskopischer Vergleich der Veränderung des Schmelzes.

Med. Diss. Freiburg i. Br. 1995

Haywood van B, Heymann HO (1991):

Nightguard vital bleaching: how safe is it?

Quintessence Int 22, 515-523

\section{Haywood van B, Leech T, Heymann HO, Crumpler D, Bruggers K (1990):}

Nightguard vital bleaching: effects on enamel surface texture and diffusion.

Quintessence Int 21, 801-04 
Haywood van B, Leonard RH, Nelson CF, Brunson WD (1994):

Effectiveness, side effects and long term status of nightguard vital bleaching.

J Am Dent Assoc 125, 1219-1226

Heidemann:

Kariologie.

4. Aufl., Urban und Schwarzenberg, München 1999

(ISBN: 3437054309)

Hellwig E, Klimek J, Attin T:

Einfürung in die Zahnerhaltung.

4. Aufl., Urban und Fischer Verlag, München 2006

(ISBN: 3437050516)

\section{Höhling HJ:}

Bauelemente von Zahnschmelz und Dentin aus morphologischer, chemischer und struktureller Sicht.

Hanser Verlag, München 1966

(ASIN: B0000BRN1X)

Howell RA (1980):

Bleaching discoloured root-filling teeth.

Br Dent J 148, 159-62

http://shop.colourconfidence.com/product.php?xProd=1822\& $\times$ Cmd=cl\&xLang=5

http://upload.wikimedia.org/wikipedia/commons/thumb/d/dc/Schematic_section_tooth. svg/150px-Schematic_section_tooth.svg.png

\section{Hupfauf L, Nolden R:}

Ästhetik in der Zahn-, Mund-, Kieferheilkunde.

PdZspezial - Kapitel "Bleichen von Zähnen”.

Urban \& Fischer Verlag, München 1995

(ISBN: 3541186119)

Joiner A (2006):

The bleaching of teeth: a review of the literature. 
J Dent $\underline{34(7)}, 412-29$

Josey AL, Meyers IA, Romaniuk K, Symons AL (1996):

The effect of a vital bleaching technique on enamel surface morphology and the bonding of composite resin to enamel.

J Oral Rehabil $\underline{23}, 244-50$

Kielbassa AM, Zantner C (2001):

Zur Einordnung der Bleichtherapie im zahnärztlichen Behandlungsspektrum.

Quintessenz $\underline{52(11)}, 1105-1113$

Kielbassa AM, Beheim-Schwarzbach NJ, Neumann K, Nat R, Zanter C (2009):

In vitro comparison of visual and computer aided pre- and post tooth shade determination using various home bleaching procedures.

J Prosthet Dent 101(2), 92-100

\section{Kim ST, Abrott PV, Mc Ginley P (2000):}

The effects of Ledermix paste on discolouration of masture teeth.

Int Endod J $\underline{33}, 233-7$

\section{Klimek J, Hellwig E, Ahrens G (1982):}

Fluoride taken up by plaque, by the underlying enamel and by clean enamel from three flouride compounds in vitro.

Caries Res $\underline{16}, 156-161$

Knösel M, Attin R, Jung K, Brunner E, Kubein-Meesenburg D, Attin T (2009):

Digital image color analysis compared to direct dental CIE colorimeter assessment under different ambient conditions.

Am J Dent 22(2), 67-72.

Lee JH, Kim HI, Kim KH, Kwon YH (2002):

Effect of bleaching agents on the fluoride release and micro hardness dental materials.

J Biomed Mater Res $\underline{63}, 535-41$

\section{Leonard RH (2000):}

Nightguard vital bleaching: dark stains and long-term results.

Compend Contin Educ Dent Supp 28, 18-27 
Leonard RH, Haywood van B, Phillips C (1997):

Risk factors for developing tooth sensitivity and gingival irritation associated with nightguard vital bleaching.

Quintessence Int $\underline{28}, 527-34$

Lips M (2004):

Blondierte Zähne.

Zahnärztl Mitt 14, 32

Luk K, Tam L, Hubert M (2004):

Effect of light energy of peroxide tooth bleaching.

J Am Dent Assoc 135, 194-201

Maibach-Nagel, M (2000):

Sehr wichtig: schöne Zähne.

Zahnärztl Mitt $\underline{90}$, 24-26

Maties BA, Hamdan YS, Cochran MA, Eckert GJ (2002):

A clinical evaluation of a bleaching agent used with and without reservois.

Oper Dent 27, 5-11

Mellberg JR (1992):

Hard-tissue substrates for evaluation of cariogenic and anti-cariogenic activity in situ.

J Dent Res $\underline{71}$, 913-919

Mielczarek A, Klukowska M, Ganowicz M, Kwiatkowska A, KwaśnyM (2008):

The effect of strips, tray and office peroxide bleaching systems on enamel surface in vitro.

Dent Mater 24(11), 1495-500

Musil R, Kittler H (1983):

Ein Beitrag zur Objektivierung der Farbbestimmung in der Prothetik.

Dtsch Zahn Mund Kieferheilkd Zentralbl 71, 477-484

Nathoo SA (1997):

The chemistry and mechanisms of extrinsic and intrinsic discoloration.

J Am Dent Assoc 128, 6-10 


\section{O'Brien WJ, Groh CL, Boenke KM (1989):}

A one-dimensional colour order system for dental shade guides.

Dent Mat $\underline{5}, 371-74$

Okubo SR, Kanawati A, Richards MW, Childress S (1998):

Evaluation of visual and instrument shade matching.

J Prosthet Dent $\underline{80}, 642-48$

\section{Pascher DD :}

Zwei-Jahres-Untersuchung dreier verschiedener kommerzieller Homebleaching-Produkte in Bezug auf Bleichwirkung und Wirkdauer in vivo.

Med. Diss. München 2004

\section{Paul S, Peter A, Pietrobon N, Hämmerle CHF (2002):}

Visual and Spectrophotometic Shade Analysis of Human Teeth.

J Dent Res $\underline{\text { 81, 578-82 }}$

Plasschaert AJM, van der Burgt TP (1988):

Zahnverfärbungen durch endodontische Materialien.

Stomatol DDR $\underline{38}, 161-166$

Plein T (2008):

Wirksamkeit von Bleichprodukten.

Dtsch Zahnärztl Z $\underline{63}, 404-17$

Potocnik I, Kosec L, Gaspersic D (2000):

Effect of $10 \%$ carbamid peroxid bleaching gel on enamel microhardness, microstructure and mineral content.

J Endod 26, 203-206

Reinhard JW, Eivins SE, Swift EJ Jr, Denehy GE (1993):

A clinical study of nightguard vital bleaching.

Quintessence Int 24, 379-84

\section{Reis M (1995):}

Farbe und Ästhetik in der restaurativern Zahnheilkunde. 


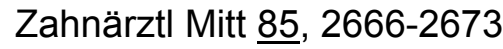

Robertello FJ, Dishman MV, Sarrett DC, Epperly AC (1999):

Effect of home bleaching products on mercury release from an admixed amalgam.

Am J Dent 12, 227-30

Rosentritt M, Lang R, Plein T, Behr M, Handel G (2003):

Das Bleichen von Zähnen.

Quintessenz $\underline{54}, 255-260$

Rotstein I, Zalkind M, Mor C, Tarabeah A, Friedmann S (1991):

In vitro efficacy of sodium perborate preparations used for intracoronal bleaching of discoloured non-vital teeth.

Endod Dent Traumatol 2, 23-26

Rotstein I, Dogan H, Avron Y, Shemesh H, Mor C, Steinberg D (2000):

Protective effect of Copalite surface coating on mercury release from dental amalgam following treatment with carbamid peroxide.

Endod Dent Traumatol 16, 107-10

Russe ND, Smith DC, Torneck CD, Titley KC (1990):

Preliminary surface analysis of etched, bleached and normal bovine enamel.

J Dent Res $\underline{9}, 1610-13$

Sarrett (2002):

Tooth whitening today.

J Am Dent Assoc 133(11), 1535-38

\section{Schroeder HE:}

Orale Strukturbiologie.

4. Aufl., Thieme Verlag, Stuttgart 1992

(ISBN: 313540904X)

\section{Schroeder HE:}

Pathobiologie oraler Strukturen.

3. Aufl., Karger Verlag, Basel 1997

(ISBN: 3805562861) 


\section{Schroeder HE:}

Orale Strukturbiologie.

5. Aufl., Thieme Verlag, Stuttgart 2000

(ISBN: 3135409058)

\section{Setz J, Geis-Gerstorfer J, Weber H (1992):}

Objektive Zahnbestimmung/Fehlerquellen werden ausgeschaltet.

Dental-labor XL $\underline{4}, 667-70$

\section{Severcan F, Gokuman K, Dogan A, Bolay S, Gokalp S (2008)}

Effects of in-office and at-home bleaching on human enamel and dentin an in vitro application of Fourier transform infrared study.

Appl Spectrosc $\underline{62(11)}, 1274-79$

\section{Sproull RC (1973):}

Colour matching in dentistry Part II. Practical application of the organization of colour.

J Prosthet Dent $\underline{29}$, 556-566

\section{Stöckli PW, Ben-Zur ED:}

Zahnmedizin bei Kindern und Jugendlichen.

Georg Thieme Verlag, Stuttgart 1994, 155

(ISBN: 3135302032)

Strub JR, Türp JC, Witkowski S, Hürzeler MB, Kern M (a):

Curriculum Prothetik.

Bd I, Quintessenz Verlags-GmbH, Berlin 2005

(ISBN: 3876524342)

Strub JR, Türp JC, Witkowski S, Hürzeler MB, Kern M (b):

Curriculum Prothetik.

Bd II, Quintessenz Verlags-GmbH, Berlin 2005

(ISBN: 3876524393)

\section{Sulieman M (2005):}

An overview of bleaching techniques: 2. Nightguard Vital Bleaching and non-vital bleaching.

Dent Update $\underline{32}, 39-46$ 
Tam L (1999):

The safety of Home Bleaching techniques.

J Can Dent Assoc $\underline{65}$, 453-55

Thitinanthapan W, Satamanont P, Vongsavan N (1999):

In vitro penetration of the pulp chamer by three brands of carbamid peroxide.

J Esthet Dent 11, 159-64

Tredwin CJ, Naik S, Lewis NJ, Scully C (2006):

Hydrogen peroxide tooth-whitening (bleaching) products: review of adverse effects and safety issues.

Br Dent J 200, 371-376

Trope M (1997):

Cervical root resorption.

J Am Dent Assoc 128, 56-59

Van der Burgt TP, Ten Bosch JJ, Borsboom PCF, Plaeschert AJ (1985):

A new method for matching tooth colour standards.

J Dent Res $\underline{64}, 837-41$

van der Burgt TP, Ten Bosch JJ, Borsboom PCF, Kortsmit WJPM (1990):

A comparison of new and conventional methods for quantification of tooth colour.

J Prosthet Dent $\underline{63}, 155-162$

Vissink A, S-Gravenmade EJ, Gelhard TB, Panders AK, Franken MH (1985):

Rehardening properties of mucin- or CMC-containing saliva substitutes on softened human enamel.

Caries Res $\underline{19}, 212-218$

Weyman J (1965 a):

The clinical appearences of tetracyclin staining of the teeth.

Br Dent J 118, 289-3

Weyman J (1965 b):

Tetracyclines and the Teeth. 
Practitioner $\underline{195}, 661-665$

Weiler J (2002):

Der devitale Frontzahn.

ZWP Spezial $\underline{3}, 12-14$

Whittaker DK (1983):

Structural characteristics of bovine enamel.

J Dent Res $\underline{62}$, 439-450

Wiegand A, Attin T(2002):

Internes und externes Bleichen von Zähnen - eine Übersicht.

Oralprophylaxe $\underline{24}, 153-158$

Woolverton CJ, Haywood van B, Heymann HO (1993):

Toxicity of two carbamid peroxide products used in nightguard vital bleaching.

Am J Dent $\underline{6}, 310-14$

www.haberbeck.de/pages/de/premedia/Colormanagement.html

Zanter C, Kielbassa AM (2005).:

Bleichmittel unter besonderer Berücksichtigung von OTC- Produkten.

Ästhet Zahnmed 2, 15-24

Zanter C, Derdilopolou F, Martus P, Kielbassa AM (2006):

Randomized clinical trial on the efficacy of a new bleaching lacquer for self-application.

Oper Dent $\underline{31}, 308-331$

Zenkonis R, Matis BA, Cochran MA, AI Shetri SE, Eckert GJ, Carlson TJ (2003):

Clinical Evaluation of In-Office and At-Home Bleaching Treatments.

Oper Dent 28-2, 114-121

Ziebolz D, Helms K, Hannig C, Attin T (2007):

Efficacy and oral side effects of two highly concentrated tray-based bleaching systems.

Clin Oral Invest $\underline{11}, 267-75$ 


\section{Material}

1. Isotone Kochsalzlösung (B. Braun Melsungen AG, Melsungen, Germany)

2. Clean Polish (Kerr Hawe, Bioggio, Switzerland)

3. Metallstifte $(2,5 \times 2,5 \times 25 \mathrm{~mm})$

4. Clearfil Liner Bond 2V (Kuraray Europe GmbH, Düsseldorf, Germany)

5. LuxaCore (DMG, Hamburg, Germany)

6. Canon Eos 350 D (Canon Deutschland GmbH, Krefeld, Germany)

7. Canon MR-14EX (Canon Deutschland GmbH, Krefeld, Germany)

8. Photoshop CS (Adobe Systems $\mathrm{GmbH}$, München, Germany)

9. Opalescence PF $15 \%$

Chargennummer: 6512 Lot B2FNR (Ultradent, South Jordan, USA)

10. Frasaco Stripkronen (Frasaco $\mathrm{GmbH}$, Tettnang, Germany)

11. Opalescence XtraBoost

Chargennummer: 1301 Lot B2BDR (Ultradent, South Jordan, USA)

12. QP Card 101(QPcardAB, Göteborg, Sweden) 


\section{Abbildungs- und Tabellenverzeichnis}

Abbildung 2.1:

Abbildung 2.2:

Abbildung 3.1:

Abbildung 4.1:

Abbildung 4.2:

Abbildung 4.3

Abbildung 4.4:

Abbildung 5.1:

Abbildung 5.2:

Abbildung 5.3:

Abbildung 5.4:

Abbildung 5.5:

Abbildung 5.6:

Abbildung 5.7:

Abbildung 5.8:

Abbildung 5.9:

Abbildung 5.10:

Abbildung 5.11:

Abbildung 5.12:

Abbildung 5.13:

Abbildung 5.14:

Abbildung 5.15:

Abbildung 5.16:

Abbildung 5.17:

Abbildung 5.18:

Abbildung 5.19:

Abbildung 5.20:

Abbildung 5.21:

Abbildung 5.22:

Abbildung 5.23:

Abbildung 5.24:

Abbildung 5.25:

Abbildung 5.26:

Abbildung 5.27:

Abbildung 5.28:
Anatomie eines Zahns sowie seiner umliegenden

Struktren ............................................................

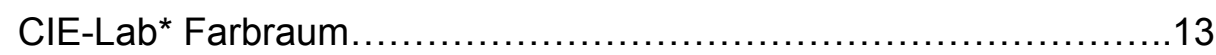

Schematische Darstellung des Versuchsablaufs.....................15

Haltevorrichtung für Kamera und Zahnprobe ........................17

Kamera und Zahnprobe........................................... 18

Zahn mit Graukartenausschnitt..................................19

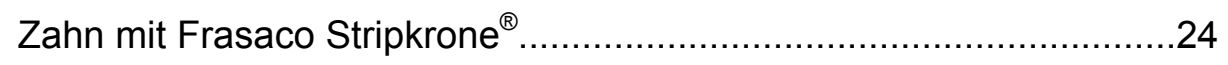

Veränderung des $L^{*}$-Wertes Baseline zu t1 .......................26

Veränderung des $a^{*}$-Wertes Baseline zu t1 ......................27

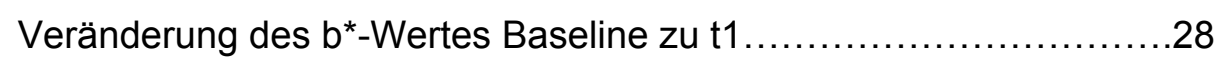

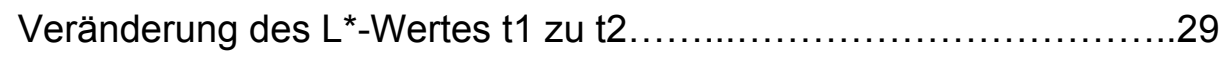

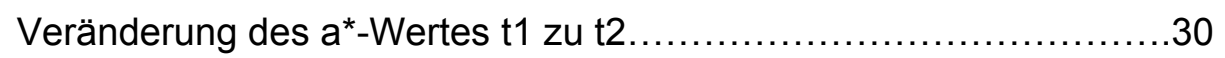

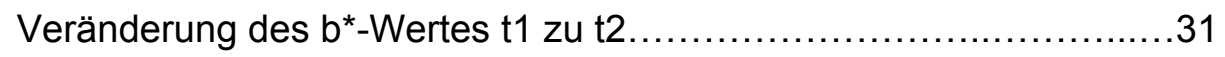

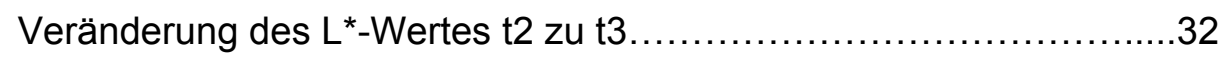

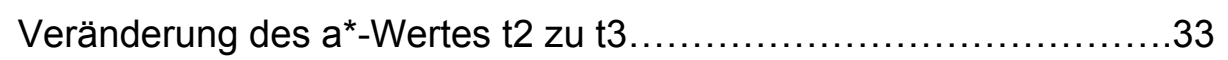

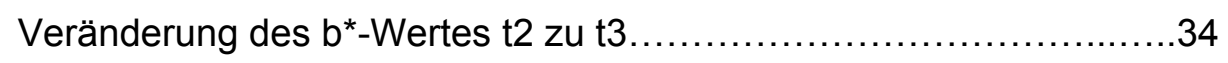

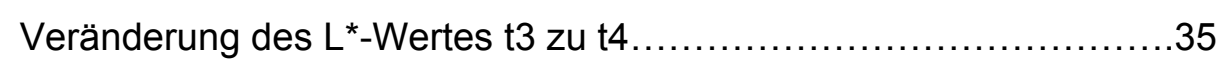

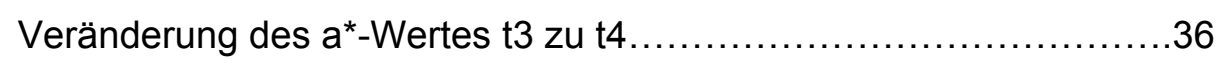

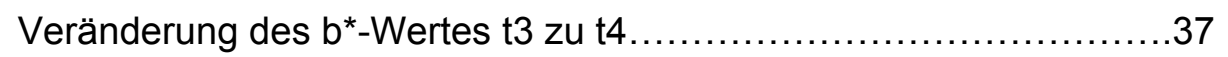

Verlauf des $L^{*}$-Wertes über die Zeit..................................39

Verlauf des $a^{*}-$ Wertes über die Zeit.................................4

Verlauf des $b^{*}$-Wertes über die Zeit...............................43

Korrelation $L^{*}$ - und $a^{*}$-Wert Zeitpunkt: Baseline......................51

Korrelation $L^{*}$ - und $a^{*}$-Wert Zeitpunkt: $t 1 \ldots \ldots \ldots \ldots \ldots \ldots \ldots \ldots \ldots \ldots . \ldots 1$

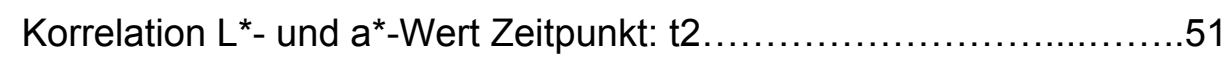

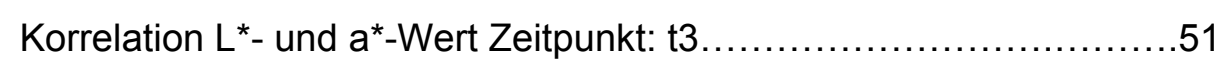

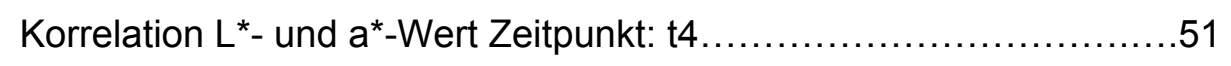

Korrelation $L^{*}$ - und $b^{*}$-Wert Zeitpunkt: Baseline.......................53

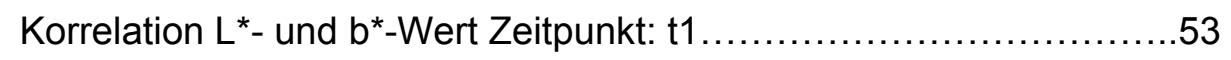

Korrelation $L^{*}$ - und $b^{*}$-Wert Zeitpunkt: $t 2 \ldots \ldots \ldots \ldots \ldots \ldots \ldots \ldots \ldots \ldots . \ldots \ldots$

Korrelation $L^{*}$ - und $b^{*}$-Wert Zeitpunkt: $t 3 \ldots \ldots \ldots \ldots \ldots \ldots \ldots \ldots \ldots \ldots . \ldots \ldots$

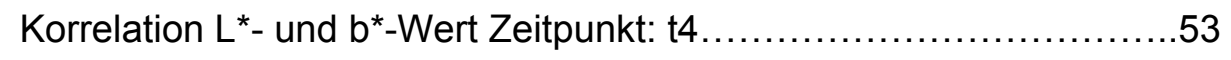

Korrelation $a^{*}$ - und $b^{*}$-Wert Zeitpunkt: Baseline.......................55

Korrelation $a^{*}$ - und $b^{*}$-Wert Zeitpunkt: $t 1$..........................55

Korrelation $a^{*}$ - und $b^{*}$-Wert Zeitpunkt: $t 2 \ldots \ldots \ldots \ldots \ldots \ldots \ldots \ldots \ldots \ldots . \ldots \ldots$ 


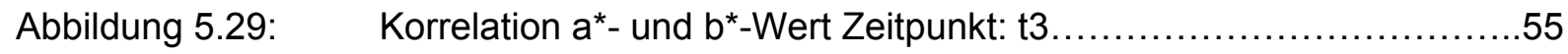

Abbildung 5.30: Korrelation $a^{*}-$ und $b^{*}$-Wert Zeitpunkt: $\mathrm{t} 4$ .55

Tabelle 4.1:

Tabelle 4.2:

Umrechnungsformel der Lab*-Werte des Computerprogramms Adobe

Photoshop CS in de Lab*-Werte der CIE nach Belngel (2003).........20

Tabelle 4.3:

Zuordnung der Lab*-Werte zu den ABCD Farben. .21

Tabelle 4.4:

$L^{*}$-Bereichseinteilung der Zähne während der Gruppenzulosung.....21

Tabelle 4.5:

Zusammensetzung des künstlichen Speichels.....................22

Tabelle 5.1:

Tabelle 5.2

Tabelle 5.3

Tabelle 5.4

Tabelle 5.5

Tabelle 5.6

Bleichintervalle der Gruppen mit aktiver Bleichsubstanz..............23

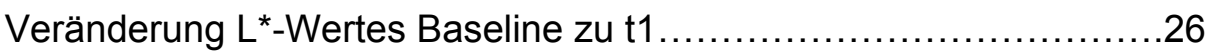

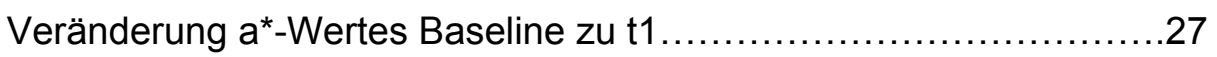

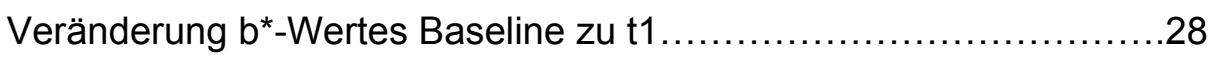

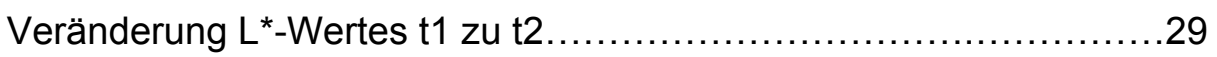

Veränderung $a^{*}-$ Wertes $\mathrm{t} 1 \mathrm{zu}$ t2 ................................... 30

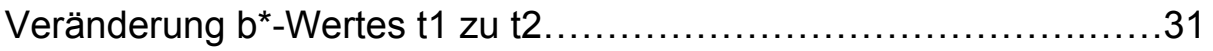

Tabelle 5.7:

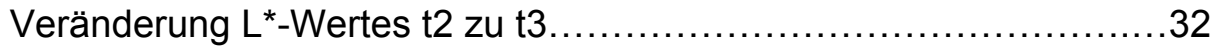

Tabelle 5.8:

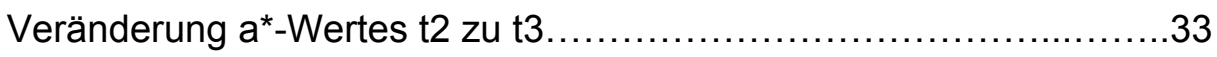

Tabelle 5.9:

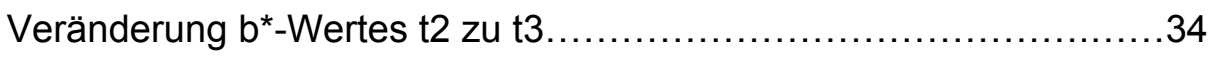

Tabelle 5.10:

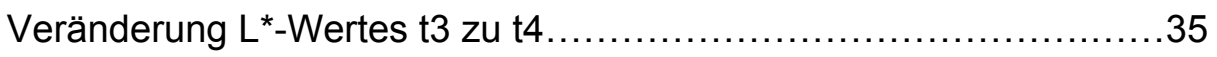

Tabelle 5.11:

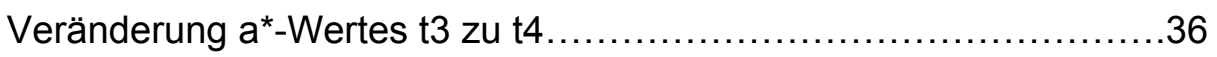

Tabelle 5.12:

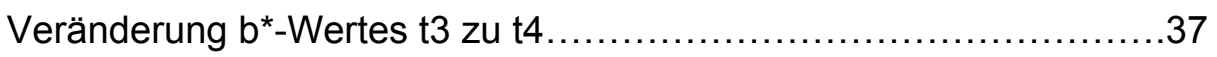

Tabelle 5.13:

Veränderung $L^{*}$-Wert Baseline zu t4................................38

Tabelle 5.14:

Veränderung $L^{*}$-Wert $\mathrm{t} 1$ zu $\mathrm{t} 4$

Tabelle 5.15:

$p$ Werte adjustiert nach Tukey zum Vergleich der $L^{*}$-Werte innerhalb

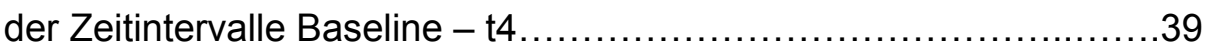

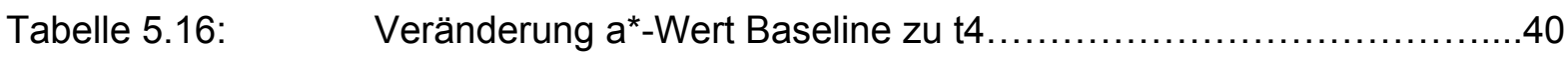

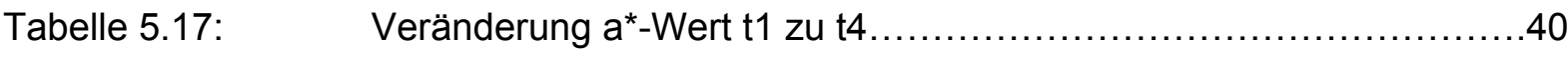

Tabelle 5.18: $\quad$ p Werte adjustiert nach Tukey zum Vergleich der $a^{*}$-Werte innerhalb

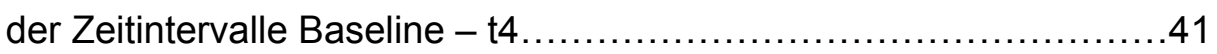

Tabelle 5.19: $\quad$ Veränderung $b^{*}$-Wert Baseline zu t4 .............................42

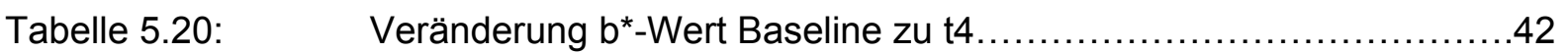

Tabelle 5.21: $\quad$ p Werte adjustiert nach Tukey zum Vergleich der $b^{*}$-Werte innerhalb

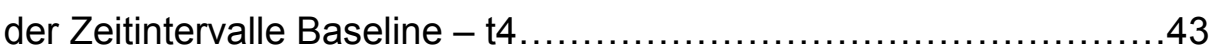

Tabelle 5.22: $\quad$ p Werte adjustiert nach Tukey zum Vergleich der $L^{*}$-Werte innerhalb der Gruppen zu den unterschiedlichen Zeitpunkten. 
Tabelle 5.23: $\quad p$ Werte adjustiert nach Tukey zum Vergleich der $a^{*}$-Werte innerhalb der Gruppen zu den unterschiedlichen Zeitpunkten.

Tabelle 5.24: $\quad$ p Werte adjustiert nach Tukey zum Vergleich der $b^{*}$-Werte innerhalb der Gruppen zu den unterschiedlichen Zeitpunkten.

Tabelle 5.25: $\quad$ Korrelation von $L^{*}$-und $a^{*}$-Werten: partielle Korrelationskoeffizienten nach Pearson.

Tabelle 5.26: Korrelation von $L^{*}$-und $b^{*}$-Werten: partielle Korrelationskoeffizienten nach Pearson. .52

Tabelle 5.27: Korrelation von $a^{*}$-und $b^{*}$-Werten: partielle Korrelationskoeffizienten nach Pearson. 


\section{Veröffentlichungen der Studienergebnisse}

1. Posterbeitrag auf der DGZ-Jahrestagung in Hannover 2009:

Monika Reus, Rainer Mausberg, Dirk Ziebolz:

Farbstabilität zwei verschiedener Zahnaufhellungsmethoden - eine in-vitro Untersuchung

2. Vortrag auf der CED-Jahrestagung in München 2009:

Michael Knösel, Monika Reus, Rainer Mausberg, Dirk Ziebolz:

In-vitro Longevity of Bleaching Results achieved by 15\% Carbamid-Peroxide and $38 \%$ Hydrogenperoxide - A Randomized Controlled Trial

3. $\quad$ Artikel eingereicht bei Journal „Clinical Oral Investigation“:

Michael Knösel, Monika Reus, Albert Rosenberger, Sven Rinke, Rainer Mausberg, Thomas Attin, Dirk Ziebolz: Longevity of

Bleaching Results achieved by $15 \%$ Carbamid-Peroxide and $38 \%$ HydrogenPeroxide - A Randomized Controlled Trial

4. Vortrag auf der regionalen zahnmedizinischen Fortbildungsveranstaltung der Bundeswehr in Hannover 2009:

Monika Reus, Rainer Mausberg, Dirk Ziebolz:

Vor- und Nachteile zwei verschiedener Zahnaufhellungsmethoden 


\section{Danksagung}

Für die freundliche Vergabe dieser Doktorarbeit möchte ich mich bei Herrn Prof. Dr. med. dent. Rainer Mausberg, Abteilung Präventive Zahnmedizin, Parodontologie und Kariologie der Georg-August-Universität Göttingen, sehr herzlich bedanken.

Ein besonderer Dank gilt Herrn Dr. med. dent. Dirk Ziebolz, Abteilung Präventive Zahnmedizin, Parodontologie und Kariologie der Georg-August-Universität Göttingen, der mir bei der Planung und Durchführung dieser Arbeit stets hilfreich zur Seite stand.

Herrn Albert Rosenberger, Abteilung genetische Epidemiologie der Georg-August-Universität Göttingen, danke ich für die statistische Auswertung.

Abschießend möchte ich mich bei den vielen Praxen, die mich mit der nötigen Menge an Zähnen versorgten, für das eifrige Sammeln bedanken. 


\section{Lebenslauf}

Mein Name ist Monika Maria Reus. Ich wohne im Oberwallweg 7, 31675 Bückeburg. Ich wurde am 07.01.1983 in Fulda geboren. Von 1989 bis 1993 besuchte ich die Bonifatiusgrundschule in Bad Salzschlirf. 1999 absolvierte ich die Mittlere Reife an der Realschule Marianum in Fulda. Von August 1999 bis Juli 2000 verbrachte ich ein Jahr als Austauschschülerin in St. Louis, Missouri, USA und besuchte dort die örtliche High-School Parkway North. Die allgemeine Hochschulreife erlangte ich im Juli 2003 am Gymnasium Marianum in Fulda. Im Oktober 2003 nahm ich das Studium der Zahnheilkunde an der Georg-August-Universität Göttingen auf und beendete es mit der zahnärztlichen Prüfung im Dezember 2008.

Seit Dezember 2008 bin ich als Zahnärztin bei der Bundeswehr tätig. 